\title{
Sleep is required for odor exposure to consolidate memory and remodel olfactory synapses.
}

Fernando Muñoz-Lobato ${ }^{1,7}$, Rashmi Chandra1,7, Fatima Farah ${ }^{2,7}$ Anirudh Bokka',7, Kelli L. Benedetti ${ }^{1,7}$, Chantal Brueggemann ${ }^{1}$, Fatema Saifuddin ${ }^{1}$, Sarah K. Nordquist ${ }^{1,3}$, Joy Li ${ }^{2}$, Eric Chang ${ }^{2}$, Aruna Varshney², Vanessa Jimenez ${ }^{2}$, Anjana Baradwaj ${ }^{2}$, Kristine Andersen², Julia M. Miller ${ }^{1}$, Ray L. Dunn ${ }^{1,3}$, Bryan Tsujimoto², Alan Tran², Alex Duong², Carlos Zuazo ${ }^{1}$ Rebekka Paisner ${ }^{1}$, Matthew A. Churgin ${ }^{4,5}$, Chris Fang-Yen ${ }^{4,5}$, Martina Bremer6, Saul Kato ${ }^{3}$, Miri K. VanHoven ${ }^{2,8^{*}}$, and Noëlle D. L’Étoile ${ }^{1,8^{\star}}$

1Department of Cell and Tissue Biology, University of California, San Francisco, San Francisco, CA 94143, USA

2Department of Biological Sciences, San José State University, San José, CA 95192, USA

3Department of Neurology, University of California, San Francisco, San Francisco, CA 94158, USA

${ }^{4}$ Department of Bioengineering, School of Engineering and Applied Science, University of Pennsylvania, Philadelphia, PA 19104, USA

${ }^{5}$ Department of Neuroscience, Perelman School of Medicine at the University of Pennsylvania, Philadelphia, PA 19104, USA

${ }^{6}$ Department of Mathematics and Statistics, San José State University, San José, CA 95192, USA

7 Indicates co-first authors

8 Indicates co-corresponding authors

*Correspondence: noelle.letoile@ucsf.edu, miri.vanhoven@sjsu.edu

\section{Summary}

Sleep is conserved across phyla and is shown here to be required for memory consolidation in the nematode, C. elegans. However, it is unclear how sleep collaborates with experience to change specific neurons and associated synapses to ultimately affect behavior. C. elegans neurons have defined synaptic connections and described contributions to specific behaviors. We show that spaced odor-training induces long-term memory, which transits a labile period before being stably maintained. This post-training labile period is required for long-term memory. Memory consolidation, but not acquisition, requires a single interneuron, AIY, which plays a role in odor-seeking behavior. We find that sleep and conditioning mark inhibitory synaptic connections between the butanone-sensing AWC neuron and AIY to decrease synapses and it is in the post-sleep wake phase that memory-specific synaptic changes occur. Thus, we demonstrate in the living organism how sleep initiates events lasting beyond the period of sleep to drive memory consolidation.

\section{Introduction}

The benefit of sleep to memory has been appreciated since antiquity. Writing in $95 \mathrm{CE}$, the Roman rhetorician Quintilian mused "...the interval of a single night will greatly increase the strength of the memory...Whatever the cause, things which could not be recalled on the spot are easily coordinated the next day, and time itself, which is generally accounted one of the causes of forgetfulness, actually serves to strengthen the memory (Quintilian, Inst. Orat. 11.2.43, trans. Butler, 
1921)." Memory provides a survival benefit: an organism that encounters pathogens emitting an environmental cue such as an odorant, and remembers the odorant is associated with a negative outcome will be more likely to avoid the pathogens and survive to produce progeny. Mammals, birds, and insects all overwrite positive associations with negatives ones and the stability of these newly formed memories depends on sleep. Some of the mystery of why sleep is so widespread across phyla could be answered by its ability to overwrite memories and thus, provide a survival benefit for those organisms that learn to avoid cues that signal death or would reduce their progeny's chances for survival.

Honeybees provide an example of experience-based overwriting of memory. A worker honeybee can be trained by just one pairing of an odorant with sugar to extend her proboscis in response to odor presentation. This positive association does not require sleep. After the bee links odor to the reward, she can be trained to ignore the smell if it is repeatedly paired with water. If she is deprived of sleep, she cannot overwrite her behavior and remains attracted to the fruitless odorant (Hussaini et al., 2009). The consequences of failing to overwrite the positive association is a lower energy yield for the hive (Beyaert et al., 2012). Male starlings also require sleep to remember a go/no-go task in which they are trained to peck for a reward if they hear a specific song, but not in response to a different one. They cannot learn to update this task if they do not sleep after training (Brawn et al., 2018). Humans also require REM sleep to consolidate memories, especially of complicated tasks, including complex declarative memories (e.g. meaningless sentences and stories, Rasch and Born, 2013). Classical studies have shown that in each example of sleep dependent learning, acquisition, consolidation, and recall depend heavily on different sets of brain regions (Vorster and Born, 2015). We still lack a molecular understanding of how and when different brain regions are recruited for each memory task (Asok et al., 2019), nor do we understand why recruitment requires sleep.

Unraveling how sleep affects cells and circuits to consolidate memory requires knowing what cells to focus on. Optimally, we could identify individual cells with defined connections that play a role in circuits that regulate thought or behavior, and identify how the structure of these circuits are altered by sleep to "hold" memories. A large body of work supports the role of two mechanisms acting together in memory consolidation: systems-level consolidation and synaptic downscaling (Tononi and Cirelli, 2014). Work in mammals, birds, and insects has identified populations of cells within specific brain regions that are essential for memory acquisition, maintenance, and recall (Yonelinas et al., 2019; Ryan et al., 2015; Abdou et al., 2018; Cowansage et al., 2015). By targeting specific brain 
regions to tag cells that are active during fear conditioning with optically-activated channels, fearbased freezing can be triggered by light. These cells are termed engram cells and they are found to initially connect cortical areas associated with the stimulus to limbic areas connected to the expression of fear (Ryan et al., 2015; Abdou et al., 2018; Cowansage et al., 2015). Over time, more cortical regions seem to be recruited to a memory trace (Asok et al., 2019). Fly and bee olfactory memories are similarly acquired in one location, the antennal lobes, and stored in the mushroom bodies, while bird song memory and chick imprinting are acquired in the intermediate and medial mesopallium and seem to be stored in the brain region S' (Vorster and Born, 2015; Menzel et al., 2006). The pattern of consolidation shares features across phyla: the memory is initially fragile, it may be maintained remotely from the region in which it is required, and it is bolstered by sleep. However, the identity of cells that participate in the memory trace, whether they are a mix of both excitatory and inhibitory, is not known with enough detail and precision to understand how sleep affects their ability to encode or hold a memory.

The properties of cells whose plasticity is required for learning and memory revolve around their ability to connect to the rest of the circuit and brain. Chemical synapses between these cells have been the focus of study for decades. Sleep may strengthen memory by modifying synaptic structures. How it does this is at present unclear. Across many regions in the mammalian (Diering et al., 2017; Dudai et al., 2015) and fly brains (Tononi and Cirelli, 2014), sleep diminishes synapse size and strength. By contrast, long-term memory (LTM) studies have shown a requirement for synaptic strengthening. The classical view of memory consolidation is that purely synaptic mechanisms such as Hebbian plasticity drive learning. Briefly, Hebbian plasticity has been the focus of long-term memory: synapses are strengthened when the pre- and post-synaptic neuron fire in synchrony and, by contrast, weakened when their firing is asynchronous. More recently, the cellular basis for Hebbian plasticity has been revealed by the observation that synchronous firing will lead to long term potentiation (LTP) while firing in an asynchronous manner induces long term depression (LTD) of a synapse. These changes lead to increases (LTP) or decreases (LTD) in the gain of the synapse, thus driving learning and memory (Fox and Stryker, 2017). Though LTP and LTD could explain memory, the field was left with a conundrum: LTP would "feed forward" into ever increasing synaptic strength and by contrast, LTD should extinguish neuronal responses. It was found that synapses are scaled such that each neuron maintains its baseline firing rate after LTP or LTD protocol. Thus, this homeostatic mechanism returns a system to its initial set point through compensatory mechanisms (often described by average neuronal firing rate) (Turrigiano et al., 1998; Turrigiano, 2017). Homeostatic plasticity can occur via scaling the synaptic strength, changes in inhibition or in intrinsic 
membrane properties, and by returning synapse size to baseline (Fox and Stryker, 2017). Memory consolidation at the synaptic level recently has been broadened to account for homeostatic scaling responses and the balance between this and Hebbian plasticity may be how sleep acts on learning and memory. Sleep has been posited to integrate these two forms of plasticity to drive memory formation by differentially affecting (down or upscaling) synapses that were acted upon in the wake state. However, the role of sleep at the synapse that holds the memory remains unknown (Tononi and Cierlli, 2014).

Across phyla, synapses in many regions are increased in the wake state and decreased with sleep. For example, in the rodent, the wake state increased synapses and sleep reduced them in the sensorimotor, primary motor, primary somatosensory, and prefrontal cortices (Maret et al., 2011; De Vivo et al., 2017; Acosta-Peña et al., 2015), and at the dendritic spines throughout the hippocampus (Kreutzmann et al., 2015; Raven et al., 2018). Structural analysis of dendritic branches in the adolescent mouse cortex have demonstrated a wake-induced increase in number and size of synapses that decreases post sleep. Sleep was shown to be necessary for these changes in synaptic plasticity (Maret et al., 2011). A role for sleep in decreasing synapse size or number has been demonstrated in three Drosophila neuron groups (small ventral lateral neurons, gamma neurons of the mushroom bodies, and the first giant tangential neuron of the lobula plate vertical system) (Bushey et al., 2011). Similarly, in mouse motor and sensory cortices, $\sim 80 \%$ of synapses were reduced in mice that were allowed to sleep, when compared with animals that were not permitted to sleep (deVivo et al., 2017). Taken together, these studies suggest that the temporal segregation of synapse building during wake via Hebbian plasticity and synapse reduction in sleep by homeostatic means allowed a reconciliation of the conundrum of how these two forms of plasticity may work (Tononi and Cirelli, 2014).

Though memory consolidation requires sleep, the global downscaling of synapses during sleep would seem to negate the effects of learning acquired by the waking animal. The synaptic homeostasis hypothesis (SHY) proposes that sleep restores synaptic homeostasis from the wake state (Born and Feld, 2012; Tononi and Cirelli, 2014; Vyazovskiy and Faraguna, 2014). There is much evidence in support of the SHY hypothesis (Vyazovskiy et al., 2008; Liu et al., 2010; Appelbaum et al., 2010; Gilestro et al., 2009; Donlea et al., 2009), including studies in flies and mouse that showed a requirement for sleep to drive homeostatic plasticity (Bushey et al., 2011; deVivo et al., 2017). The SHY hypothesis originally was evoked to explain how slow-wave (non-REM) sleep, the sleep cycle required for memory consolidation in humans, induces a global downscaling of 
synapses in the hippocampus, neocortex, and olfactory cortex after the upscaling of synapses following information acquisition and learning during the wake state (Yamaguchi, 2017; Walker, 2009). The question the SHY hypothesis raises is how does downscaling of synapses during sleep not block learning-dependent changes at the synapse? One answer is that downscaling may act differentially on synapses that have undergone LTP versus synapses that have undergone LTD during wake, thus allowing memories to be retained during sleep. Interestingly, Hengen et al., 2016 found that neuronal firing rate homeostasis was suppressed during sleep and promoted by wake and enhanced by a longer wake state in mouse visual cortical neurons. Thus, the mechanisms by which sleep acts on cells and synapses that store memories are still largely unknown. In order to answer this question, we need cellular and synaptic resolution of memory and how it is affected by sleep.

How sleep affects memory consolidation by targeting specific synapses between individual cells with known functions in a behaviorally-relevant circuit remains unknown in any system. Though invaluable research in sleep and long-term memory has been performed in complex systems, e.g. mouse, humans, and insects, the complexity of these systems has precluded an understanding of the interplay of how sleep drives memory formation with single-cell and synaptic resolution.

Caenorhabditis elegans (C. elegans), with its behavioral robustness and complete connectome description of the circuits that underlie these behaviors, is a more tractable system in which to study how sleep affects synapses. The compact nervous system of the $C$. elegans hermaphrodite is mapped and invariably has 302 neurons (White et al., 1986; Cook et al., 2019) and functions have been assigned to most of these neurons (Bargmann and Marder, 2013). Despite its simplicity, $C$. elegans displays a wide repertoire of complex behaviors such as associative learning, memory, and sleep (Ardiel and Rankin, 2010; Trojanowski and Raizen, 2016). Moreover, a plethora of powerful tools are available for the study of the nervous system of this nematode: its transparency allows visualization of both the whole brain (Nichols et al., 2017) as well as individual synaptic connections in live animals (Feinberg et al., 2008), monitoring of neuronal activity (Chronis et al., 2007) and optogenetic perturbation of specific neurons (Nagel et al., 2005), along with the study of behavioral outputs to determine causal relationships. Appealingly, since we can visualize specific synaptic connections in live animals, we can study which exact synapses are modulated by experience and sleep.

Importantly, the behavioral properties of sleep: reduced locomotion (Hill et al., 2014; Raizen et al., 2008), stereotypical posture (Schwarz et al., 2012; Tramm et al., 2014), increased arousal threshold (Hill et al., 2014; Raizen et al., 2008), homeostatic response to deprivation (Nagy et al., 
2014; Raizen et al., 2008) and the molecular pathways that control sleep are conserved across phyla, including in nematodes and mammals (Trojanowski and Raizen, 2016; Zimmerman et al., 2008). For instance, EGF and GABA promote sleep, Period genes control its timing, PKG activity regulates its intensity, while PDF and dopamine govern waking in all worms, mammals and flies (Van Buskirk and Sternberg, 2007; Choi et al., 2013; Singh et al., 2014; Trojanowski and Raizen, 2016). However, no association between sleep and memory consolidation has been described for $C$. elegans as of yet (Trojanowski and Raizen, 2016).

We examine the well-described olfactory system using a spaced-training paradigm in which the odorant butanone is paired with starvation. The worm goes from seeking out a point source of butanone to avoiding or ignoring it. Butanone is produced both by nutritious and pathogenic strains of bacteria and thus, the worm needs to overwrite its response to this volatile cue as a function of its experience. The paradigm we use to overwrite $C$. elegans attraction to butanone is a repeated spaced training that, remarkably, is followed by sleep. This task is akin to the honeybee extinction training and the go/no-go training of starlings. We adapted this from Kaufmann et al., 2010 by pairing butanone with a negative experience. Here we show that the memory is labile for two hours after training. During this time, sleep is required to consolidate the memory into a stable form. The labile memory requires a dispersed set of at least two interneurons, but is consolidated into long-term memory by the AIY interneuron pair. We show that sleep during this consolidation period is required to reduce inhibitory synaptic connections between AWC and AIY. Thus, the mechanism by which sleep consolidates an overwritten memory is by removing synaptic connections in an odor-seeking circuit. We find that sleep does this by acting on a signal that is established during conditioning. Sleep then initiates processes that continue past the period during which the memory is fragile, to reduce synapses. This provides cell biological insight into how experience, when followed by sleep, triggers physical changes in the circuit hardware at the level of the connections between neurons. Our time course shows that the newly acquired information, specifically, that butanone is deleterious, is held in a dispersed neuronal network where it is dynamic and easily overwritten. If the animal is allowed to sleep, then physical reductions of the synaptic connections commit the circuit to a stable change. Thus, information is held, manipulated, and stored in an intact nervous system during the memory labile period when the synapses are decreased after training and sleep. This provides a testable hypothesis for why sleep is strongly evolutionarily selected for: if an organism cannot update its response to a cue that could kill it before it reproduces, it will be selected against. 


\section{Results}

\section{Spaced olfactory conditioning yields long-lasting memory}

In order to understand how long-lasting memories are formed and retained, we designed a Pavlovian style conditioning paradigm to quantitate the strength and duration of an olfactory memory. Unconditioned $C$. elegans animals move towards butanone, a volatile component of naturally attractive bacterial food sources (Worthy et al., 2018a; Worthy et al., 2018b), which they sense using their AWC ${ }^{O N}$ olfactory sensory neuron (Wes and Bargmann, Nature 2000). We quantify this attraction by employing the population-based chemotaxis assay developed by Bargmann et al. in 1993. In this assay, animals are placed onto a $10 \mathrm{~cm}$ petri dish filled with a layer of agar and a point source of diluted butanone is placed opposite a similar source of diluent (ethanol) and animals are introduced at an origin equidistant from each source. Each spot is supplemented with sodium azide to paralyze the animals once they reach it. After at least two hours of roaming, the position of each animal on the plate is scored. The chemotaxis index is calculated by subtracting the number of animals at ethanol from the number at butanone and dividing by the total number on each plate. The bulk of a naïve or buffer-exposed population is attracted to the odor butanone: they move towards the point source of butanone and their chemotaxis index $(\mathrm{Cl})$ is usually from 0.6 to 0.9 (Figure $\mathrm{S} 1$ ). Of note, each point on the graphs represents the $\mathrm{Cl}$ resulting from a population of $>50$ animals. Experience, however, can suppress or even reverse this attraction. Pairing butanone with a single 80-minute period of food removal causes the population to move randomly with respect to the odor and the $\mathrm{Cl}$ drops to near zero during subsequent recall tests (Colbert and Bargmann, 1995). This indifference indicates that the animals have acquired the memory that butanone is profitless. We define memory as the response of the odor-trained population (with $\mathrm{Cl}$ as the readout) being significantly different from the buffer-trained cohort. We use the conditioned response of the unperturbed wild-type to compare with the experimental groups to test if they differ significantly. If they differ, then we deem them memory defective. This memory decays rapidly and, after two hours of recovery on food, the population is once again attracted to butanone and this attraction persists for at least 16 hours (Figure S2). Previous studies using other pairings in $C$. elegans and in other organisms have shown that spacedconditioning paradigms in which the unconditioned and the conditioned stimuli are paired in different intervals separated by resting periods give rise to long-lasting memory (Estes, 1955; McGaugh, 1966; Mauelshagen et al., 1998; Beck et al., 2000; Kauffman et al., 2010). Employing a similar paradigm, we found that three spaced pairings of butanone and the absence of food with recovery periods on food in between the odor conditioning periods (Figure 1A) stably reduces attraction to butanone. This 
memory persists for at least 16 hours when animals are allowed to recover with food (Figure 1B, second pair of bars). The enduring nature of this olfactory memory indicates that the spaced-training paradigm induces a long-lasting memory and thus, we proceeded to ask whether this memory was specific for the training odor, or was a result of generalized inability to respond to odor across all sensory neurons, and whether it requires the cyclic AMP response element binding protein, CREB.

To determine if this conditioning paradigm interfered with odor detection in general, we asked if the butanone conditioning affected the animals' ability to sense and track the food-associated odor, benzaldehyde, which is an odor sensed by both AWC neurons. We found that pairing butanone with the absence of food did not affect attraction to benzaldehyde after 16 hours of recovery (Figure 1C), confirming that animals specifically remember their aversion of butanone, but keep their attraction to an odor sensed by the AWC ${ }^{\text {OFF }}$ neurons. Thus, the training does not impair the animal's general ability to chemotax.

To understand if the long-lasting memories are specific for butanone, or if this is a more generalized property of the olfactory system, we asked if repeated pairings of the AWA olfactory sensory neuron-sensed odor diacetyl (2,3-butanonedione, Sengupta et al., 1996) with the absence of food would also result in long-lasting memory (Figure S3). We found that three spaced pairings of diacetyl and the absence of food reduce the chemotaxis response to diacetyl initially after spacedtraining and the reduced attraction persists after 16 hours of recovery on food (Figure S3). This finding is in agreement with prior studies that showed that training with diacetyl decreases attraction to this odor that is partially retained after 24 hours of recovery, though some of the memory is lost (Hadziselimovic et al., 2014). We observe that butanone memory, likewise, decays between 2 and 16 hours of recovery, albeit more slowly than that of diacetyl.

CREB, the cyclic AMP response element binding protein, is required for memory formation in flies, Aplysia, mice (Silva et al., 1998), and more recently, in C. elegans appetitive learning (Kaufmann et al., 2010). We tested whether CREB also plays a role in our negative associative longterm memory paradigm by performing the butanone spaced-training with $c r h-1(t z 2) / C R E B$ mutants. Interestingly, we found that though the buffer-trained (control) populations of crh-1/CREB mutants show reduced chemotaxis to butanone, they were able to acquire memory as seen in the 0-hour recovery (Figure S4). This memory is maintained at 16 hours post recovery as the $\mathrm{Cl}$ of the wild-type and crh-1/CREB butanone-trained populations are not significantly different from each other (Figure S4). Though the difference between buffer and butanone-trained animals is less striking than in wildtype populations, we conclude that the long-term training paradigm may not require CREB. 
Thus, C. elegans can learn in a Pavlovian-style spaced-training paradigm to stably and selectively avoid at least two innately-attractive odors if they are associated repeatedly with the absence of food.

\section{Butanone memory is labile immediately post conditioning}

Learning is known to progress through stages, the first of which is labile, and thus we sought to determine the time course for stabilization of the aversive olfactory memory. A single butanone-no food pairing creates a memory that persists for just 30 minutes (Figure S2) which is in contrast to the more enduring memory engendered by three-cycle spaced interval conditioning (Figure 1A, 1B). It is of note that three-cycle trained animals strongly ignore and are even averse to butanone as compared to one-cycle trained animals, which show no preference or aversion to the odor (Figure 1B, Figure S2). To understand the dynamics of LTM decay, we assessed the chemotaxis index (CI) of three-cycle trained populations every 15 minutes following conditioning (Figure 2A). To our surprise, we found that animals are attracted to butanone when tested 15 minutes after conditioning. This apparent memory loss persists for up to 60 minutes. Memory, as indicated by Cls close to zero, returns around 75 minutes, after which point, the memory persists with only slight decay until the 16hour time point. This can perhaps be better appreciated by considering the Learning Index $(\mathrm{LI})(\mathrm{LI}=$ $\mathrm{Cl}_{\mathrm{CTL}}-\mathrm{Cl}_{\mathrm{BTN}}$ ) plotted in Figure 2B which shows the rapid decline in $\mathrm{LI}$ at 15 minutes recovering after two hours. The difference between the $\mathrm{LI}$ after two and 16-hour recovery is not as significant or great as between 0 and 15 minutes, which suggests two distinct mechanisms for memory decay. These results indicate that recently acquired memories are labile in C. elegans. Memory in insects, mammals and avians, transitions through a labile stage that stabilizes after a sleep bout (reviewed in Vorster and Born, 2015). This prompted us to examine our populations of trained animals more closely in the two hours in which memory was most labile.

\section{C. elegans animals sleep after spaced olfactory conditioning}

We noticed that animals appeared lethargic after spaced conditioning, thus we asked whether $C$. elegans sleep after conditioning. Sleep is best defined as a behavioral state in which animals are reversibly quiescent, reduce their feeding rates, take longer to arouse, and exhibit increased quiescence when they are deprived of sleep (sleep homeostasis) (Trojanoswki and Raizen, 2016). Therefore, we quantitated each sleep-related behavior in populations of buffer and butanoneconditioned animals. 
We first compared movement of the butanone-trained to untrained animals during the first hour of recovery. We placed animals into the individual wells of a WorMotel (Churgin et al., 2017), thus keeping animals separated from each other and allowing us to monitor locomotion using an automated imaging system. The output is shown in Figure 3A where blue sections of a raster plot represent 30 seconds of complete stillness. We quantitated the mean total quiescence for each population within the WorMotel and found that the mean total quiescence of trained populations is increased compared to that of the untrained animals (Figure 3B; untrained showed 5 minutes quiescence/hour vs. $\sim 10 \mathrm{~min} / \mathrm{hr}$ in the butanone-trained populations). Further, we found that whether cohorts of animals are conditioned with buffer or butanone, that their mean quiescence increases (Figure 3B; each population shows between 10 and 20 minutes of quiescence/hour). Thus, spaced training increased quiescence, regardless of whether they were buffer or butanone trained.

When we examined feeding rates, we found that they are significantly decreased in the trained populations (Figure 3C; 214 pumps/min for untrained vs 198 for trained). In addition, it takes longer for butanone-trained than untrained animals to mount an escape response when stimulated with a blue light pulse (a noxious stimulus) in conjunction with mechanical (1 KHz) vibrations from a piezo buzzer (Figure 3D; 7 seconds for untrained vs 12 for trained). The trained animals also show fewer sinusoidal waves after the stimulus is removed (Figure 3E; 7 sinusoidal waves for untrained vs 4 for trained). This reduced movement after arousal may reflect a sleep debt incurred by the stimulation, or could mean that the animals are more tired. Thus, by these criteria, animals that are conditioned with three cycles of butanone or buffer and the absence of food alternating with feeding exhibit the hallmarks of sleep.

Taken together, these findings indicate that spaced training promotes a sleep state subsequent to spaced conditioning with buffer alone or butanone. Finally, we asked if the mean total quiescence of a population immediately after training would correlate with the strength of memory after 16 hours of recovery as assessed by the $\mathrm{Cl}$. We found that there is a moderate $(r=-0.3914)$, but significant $(P$ $=0.0394$ ) correlation between our measures of sleep one-hour post training and memory strength at 16 hours (Figure S5). Thus, conditioning induces a sleep state, which correlates with the strength of memory after 16 hours of recovery. We wondered if sleep post training was also necessary for longlasting memory. 


\section{Long-term butanone memory requires sleep}

Memory consolidation at both the synapse and systems levels has been reported to require sleep in flies, mice, and humans (Tononi and Cirelli, 2014; Dudai et al., 2015). Thus, we wondered whether post-conditioning sleep is required for long-term 16-hour butanone memory in C. elegans as well. Starved C. elegans worms roam in search of food and do not sleep (Gallagher et al., 2013; You et al., 2008). We took advantage of this to keep animals from sleeping after conditioning to ask whether sleep is necessary for long-term memory. We disrupted sleep in the first two hours post conditioning, when memory appears most labile, by removing animals from food and then allowing them to recover on food for the remaining 14 hours prior to behavioral testing. We find that the animals off food immediately post conditioning are significantly less quiescent than animals on food (Figure 4A; on food is $15 \mathrm{~min} / \mathrm{hr}$ quiescence vs $3 \mathrm{~min} / \mathrm{hr}$ for off food). These data confirm that removing animals from food keeps them awake after conditioning. When we assessed memory at 16 hours post conditioning, we found that animals that were initially kept off food do not sleep and they fail to maintain memory (Figure 4B; last pair of bars, the CTL and BTN mean Cls are not significantly different from each other). This result suggested that sleep might be necessary for memory maintenance. Another interpretation is that our treatment (removal from food) induced changes in metabolism rather than lack of sleep blocking memory stabilization.

In order to rule out the purely metabolic effects of starvation, we decided to induce sleep in animals that were removed from food. We forced animals that were recovered off food to sleep by overexpressing a sleep neuropeptide. Independent neuronal circuits control different types of sleep in C. elegans (Trojanowski et al., 2015) and different neuropeptides have been shown to mediate the induction of sleep by these neurons (Nelson et al., 2013, 2014; Turek et al., 2016). The FLP-11 neuropeptide mediates developmentally-timed quiescence and has also been found to induce sleep when overexpressed (Turek et al., 2016). Overexpression of FLP-11 not only induces sleep in animals off food (Figure 4C) but also restores memory to populations that were off food post conditioning (Figure 4D; compare the two hours off food, no sleep, and no peptide fifth bar with the off food plus peptide sixth bar). Heat shock induces sleep via the stress pathway and causes the release of somnogenic peptides (Hill et al., 2014), which may explain the small increase in memory of heatshock treated wild-type animals off food (Figure 4D). The increase in memory of animals that have been forced into sleep via peptide expression while they are off food suggests that it is not purely the metabolic changes induced by starvation that block memory stabilization; rather, it is the lack of sleep. Further, restoration of sleep by overexpressing peptides that induce sleep in development or after stress indicates that memory stabilization after sleep is not restricted to a specific type of sleep, 
but may be a general feature of sleep. Thus, sleep is necessary for memory and restoring sleep to animals off food is sufficient to promote long-lasting memory. The exact cellular mechanism by which sleep acts to consolidate memory is elusive because the field lacks precise cellular resolution of the cells that encode the memory trace.

\section{Long term memory does not result from changes in AWC sensory neuron activity}

One advantage of using the transparent $C$. elegans is that we can examine neuronal activity at the single neuron level in live animals at various times points during the sleep-induced memory stabilization. The AWC sensory neuron is the entry gate for butanone detection, thus we used GCaMP3 (Tian et al., 2009) to monitor calcium transients in AWC as memory develops. We imaged animals immediately after conditioning when they are repulsed from butanone, but the memory is labile, and after 16 hours of recovery on food when they are indifferent to the odor, but the memory is stable (Figure 5).

As previously reported (Chalasani et al., 2007; Cho et al., 2016), AWC calcium levels decrease when animals are exposed to butanone and rise dramatically after odor removal before returning to baseline. However, we find that odor removal triggers a larger increase in calcium in animals immediately after three cycles of butanone training than in the buffer-trained controls (Figure 5A, $P=$ 0.0264). Odor onset triggers a small, but significant (Figure 5B, $P=0.0172$ ) silencing of the AWC neuron in butanone-trained animals as compared to the buffer-trained controls. The difference between AWC activity in buffer and butanone-trained animals is thus seen immediately after training while the animals are repulsed from the odor and the memory is labile (Figure 5A and B, respectively). However, after 16 hours recovery on food when the memory is stabilized and the animals are indifferent to butanone, these differences in the AWC response to butanone disappear (Figure 5C $(P=0.316)$ and $D(P=0.521))$. Thus, it is unlikely that the sensory response of AWC is solely responsible for maintaining memory.

\section{The long-lasting memory trace is held in the interneurons AIB and AIY}

We reasoned that the cells that hold the memory trace may thus be downstream of AWC. Serial electron micrographs (White et al., 1986; Cook et al., 2019) indicate that the primary postsynaptic partners of AWC chemosensory neurons are the AIY interneurons, which make approximately 8-12 synapses with the AWCs, and the AIA and AIB interneurons, which each make approximately 4-5 synapses with the AWCs (see Figure 6A). Thus, we killed AIB by expressing the caspase CED-3 from the odr-2b promoter specific for the neuron. To inactivate AIY, we employed the $t t x-3(k s 5)$ mutant 
allele that prevents the birth of the AIY neurons (Altun-Gultekin et al., 2001). We found that animals that lack either AIB or AIY are still able to acquire memory (Figure 6B, compare first pair to the second and third pair of bars). However, when AIB and AIY are both missing, memory acquisition is reduced (see Figure 6B, compare first and fourth pair of bars). This might be explained if another neuron in the circuit is primarily responsible for memory acquisition and AIB and AIY are redundant and less involved. Although animals missing either AIB or AIY are able to acquire the memory, animals missing AIY lose the memory after 16 hours of recovery on food (compare tenth bar with fourteenth and sixteenth). This demonstrates that the AIY neurons are required for long-lasting memory. Thus, we have identified one specific cell that is required for memory consolidation; this is to our knowledge the highest cellular precision for memory. Having identified the exact cells that hold the memory allows us to now determine the subcellular mechanism by which memory is held and how sleep may affect the memory trace.

\section{Synapses between AWC and AIY neurons are diminished after odor training and sleep}

AIY interneurons are the primary postsynaptic partners of the AWC chemosensory neurons, and their ablation causes the most severe defect in memory. To determine if the structure of AWC-AIY synapses are altered by sleep after conditioning, we utilized the split GFP-based trans-synaptic marker Neuroligin-1 mediated GFP Reconstitution Across Synaptic Partners, or NLG-1 GRASP, (Figure 7A and Feinberg et al., 2008; Park et al., 2011; Varshney et al., 2018) to label synapses between AWC and AIY (Figure 7B, Feinberg et al., 2008; Park et al., 2011; Varshney et al., 2018). We found that the localization and distribution of AWC-AIY NLG-1 GRASP fluorescent puncta (Figure 7C) is consistent with EM micrographs, as has been the case for several other neuron pairs visualized with this marker (White et al., 1986; Cook et al., 2019; Park et al., 2011; Varshney et al., 2018; Feinberg et al., 2008), and did not affect the animals' behavior (Figure S7).

We subjected AWC-AIY NLG-1 GRASP-labeled animals to three cycles of training, but we recovered half the cohorts without food for two hours. As shown in Figure 4A, under these conditions, animals fail to sleep during this time and though animals are returned to food for the 14-hour balance of the recovery time, their memory is impaired (Figure 4B). The other half of the cohort recovered on food for the entire 16 hours, allowing them to sleep in the two hours after training. We then compared AWC-AIY NLG-1 GRASP fluorescence intensity in the butanone-trained populations with the buffertrained cohorts that either slept or were kept awake post-training (Figure 7D, E). The AWC-AIY NLG1 GRASP fluorescence intensity was assessed on at least four independent days with a total $\mathrm{N}$ of greater than 90 animals for each condition. We found that the median synaptic intensity does not 
significantly differ between buffer-trained animals that are permitted to sleep and those that are not (Figure 7D, E, compare first and third boxes). Similarly, the median synaptic intensity does not significantly differ between buffer-trained and butanone-trained animals that are not permitted to sleep (Figure 7D, E, compare third and fourth boxes). By contrast, the synaptic signal in animals that were butanone-trained and permitted to sleep post-training is significantly lower than that in the buffer-trained animals permitted to sleep (Figure 7D, E, compare first and second boxes). This indicates that odor training and sleep work together to diminish AWC-AIY synapses in animals that retain the memory.

To determine if synaptic changes in response to butanone training are global, we examined the synaptic connections between PHB chemosensory neurons and two of their primary postsynaptic partners, the AVA neurons, using a strain that carries a NLG-1 GRASP marker that labels connections between this pair of neurons (Park et al., 2011; Varshney et al., 2018). PHB is a chemosensory neuron that senses noxious chemicals including dodecanoic acid (Tran et al., 2017) and sodium dodecyl sulfate (Hilliard et al., 2002). PHB-AVA connections are not significantly altered by training or sleep (Figure S6), indicating that the synaptic changes induced by butanone training and sleep are not global.

Given that both training and the two-hour labile period during which the animals sleep are required for alteration of AWC-AIY synaptic structures, synaptic changes could occur with at least three different timelines: A) AWC-AIY synaptic reductions could be specified and take place during training, and be stabilized during post-training sleep, B) synaptic reductions could be specified and occur during training and sleep, or $\mathrm{C}$ ) synaptic reductions could be specified during training and sleep, but occur later during the 14-hour recovery period. Timelines A-C can be distinguished, as in timeline A, a reduction in AWC-AIY synaptic signal in butanone-trained compared with buffer-trained animals would be observed immediately after training. In timeline B, a difference in synaptic signal would be observed only after the two-hour period of sleep. In timeline C, synaptic changes would be observed only after the 16-hour period of recovery. The timelines would suggest different roles for sleep in memory consolidation. Timeline A would suggest a maintenance role for sleep, while timelines B and C predict that sleep diminishes the synapses of interest or specifies them for subsequent reduction, respectively. To test these hypotheses, we assessed AWC-AIY NLG-1 GRASP fluorescence directly after training, after the two-hour post-training sleep, and after the subsequent 14-hour recovery period in buffer-trained and butanone-trained animals. 
When we assessed NLG-1 GRASP fluorescence directly after training, we find that the level of synaptic signal in buffer-trained and butanone-trained animals is not significantly different (Figure 7F, first and second boxes). Thus, the training itself does not reduce synapses, and timeline $A$ is unlikely to explain the observed plasticity at 16 hours post-training. After the two hour post-training period of sleep, synaptic signal was significantly reduced in both buffer-trained and butanone-trained animals compared to the same cohorts immediately after training (Figure 7F, compare first to third and second to fourth boxes), however the level of synaptic signal was not significantly different between these two populations at this time point (Figure 7F, compare third and fourth boxes). This indicates that our system can reveal rapid synaptic dynamics in a physiologically-relevant time period. This is consistent with timeline $\mathrm{C}$, in which butanone-training and sleep-induced AWC-AIY synaptic alterations are specified during training and sleep, but occur later during the 14-hour recovery period.

\section{Discussion}

Sleep is highly conserved, indicating evolutionary pressure to be retained. Here we show that C. elegans, which diverged from our last common ancestor $\sim 1.18$ billion years ago (Wang et al., 1999), is our most distant relative that requires sleep to consolidate memory. The current state of the field of learning and memory is that we have a systems understanding of engrams at the level of cells and synapses (Asok et al., 2019). Having a living, behaving animal that allows the synthesis of these processes in such a way that they can be studied with great resolution would give us insights into the molecular and cellular biology underlying memory. Here, we show that $C$. elegans forms long-lasting aversive memories to the odorant butanone following three spaced-training cycles. This olfactory memory transitions through a two-hour labile period before being stably maintained. During this labile period post training, we find that animals sleep and that sleep is required to stabilize memory. Animals kept off food during the period of time that memory is fragile do not sleep and also fail to maintain the memory. Forcing animals to sleep off food by overexpressing a sleep neuropeptide during this time, however, enables them to keep the memory. In order to understand the circuitry involved in this paradigm, we looked at calcium dynamics in the AWC olfactory sensory neurons in animals subjected to odor training. We found that though there was a difference in response to butanone in the AWC neurons in animals immediately after training while the memory was labile, this difference disappeared by 16 hours, when the memory was stable. To understand how chemotaxis behavior could diminish so profoundly when the olfactory circuit's sensory neurons are apparently still sensitive to odor, we asked if downstream interneurons might underlie changes in behavior. We found that memory could be acquired in the absence of either AIB or AIY; however, the AIY interneuron was required for long-term memory maintenance. To understand the mechanism by 
which this neuron might store the olfactory memory, we examined synapses between AWC and AIY. Interestingly, we found that synapses were decreased 16 hours after training, but only in animals that were both conditioned to butanone and had slept. To probe the mechanism behind the collaboration between olfactory conditioning and sleep, we examined the time course for training and sleepdependent synapse reduction as a function of memory. We found that synapses are reduced in animals after their memory is stable two hours post training, regardless of whether they were trained with buffer alone or buffer and butanone. This reduction occurs in the two hours of recovery on food. The difference between the AWC-AIY synapses in butanone as compared to buffer-trained animals does not become apparent until the animals have recovered for 16 hours on food. This change suggests that odor training marks the synapses between the sensory AWC and AIY interneuron for reduction and that sleep collaborates with these marks to diminish the connection further once the animal reawakens.

\section{Evolutionary selection for memory consolidation}

Memory is essential for survival; failure to heed cues associated with harmful infectious agents can be fatal. Odors, universally powerful signals for food and its contaminants, are such salient cues. Butanone is possibly one such cue for $C$. elegans. This volatile chemical, emitted from both nutritious and infectious bacteria (Worthy et al., 2018a; Worthy et al., 2018b), could thus be associated with either positive or negative experiences. For example, the pathogenic Serratia marcescens attracts $C$. elegans with its bouquet of butanone and acetone, is subsequently ingested, and infects the host, killing it in 2-3 days (Worthy et al., 2018a). However, once the bacteria are ingested and if the worm survives, it will have learned to avoid the bacteria's bouquet (Zhang et al., 2005). Thus, the mechanism for learning to ignore butanone could potentially be an evolutionary trait to avoid further ingestion of pathogenic bacteria. This may explain why C. elegans can be trained to either seek butanone (Torayama et al., 2007; Kaufmann et al., 2010; Vohra et al., 2017), or avoid it completely (Tsunozaki et al., 2008; Figure 1). In studying this memory, we are tapping into the stronglyconserved function of odor to uniquely signal discreet information about the environment. The synaptic remodeling we observed after sleep may permit increased evolutionary fitness. This refinement may allow the animal to stably and appropriately respond to salient cues such as pathogen-associated odors and be a clue for why sleep is so highly-conserved among animals that have a nervous system.

The aversive memory of butanone is stable and lasts for an ethologically-relevant length of time: 16 hours out of the 18-day lifespan of $C$. elegans, which is proportional to three years of an 
average 79 year-long human life (Kochanek et al., 2017). Further, this memory is engaged when the animal is in its prime reproductive stage (day-one adult) and accounts for more than half of its fiveday reproductive span. The initiation and duration of this memory could thus have profound consequences for the survival of the animals' progeny. This evolutionary argument may explain why stable learning after spaced training is seen across phyla. We posit that the circuit, cellular, and molecular logic underling these processes is also conserved, and that we have developed a behavioral correlate to memory overwriting in $C$. elegans that has opened up this organism to indepth understanding of long-term memory driven by sleep.

Memory is consolidated by sleep and its benefit may serve an evolutionarily-conserved function. Individuals that sleep after an adverse encounter with a noxious agent may thus link the negative experience with the salient cue that is removed when they can finally sleep. Though this is the first report of the $C$. elegans nematode sleeping after spaced-training, sleep has been studied for over ten years in C. elegans. Developmental sleep, or lethargus (Raizen et al., 2008), was initially discovered, followed by stress-induced sleep in the adults (van Buskirk and Sternberg, 2007). More recent studies found that starvation also induces sleep in worms. Starvation causes a stress-induced sleep that is linked to inhibited insulin signaling and was posited to reflect an energy conservation mechanism (Skora et al., 2018). These prior studies documented in C. elegans the hallmarks of sleep that are conserved across the animal kingdom: periods of quickly reversed immobility, increased arousal threshold, homeostatic compensation, and stereotypical posture (Trojanowski and Raizen, 2016). With this report, we show that like other organisms, nematodes require sleep to consolidate memory. This may explain the strong evolutionary conservation of sleep among animals that have a nervous system: to modify their behavior as a function of experience.

\section{Sleep post conditioning}

Stress may induce sleep after conditioning. C. elegans and other organisms sleep in a variety of circumstances - growth stages are coincident with increased sleep needs in adolescent mammals as well as before each molt in $C$. elegans larval development. Mammals and nematodes sleep after UV light-induced DNA damage (Trojanowski and Raizen, 2016), prolonged starvation (Skora et al., 2018), re-feeding after starvation (You et al., 2008), and heat shock (Hill et al., 2014). Each trigger engages neurons in a sleep circuit that release somnogenic peptides (Trojanowski and Raizen, 2016). C. elegans' ALA interneuron triggers stress-induced sleep by releasing the FMRFamide FLP13 among other neuropeptides (Nath et al., 2016) and the interneurons RIS and RIA regulate lethargus at least in part by releasing FLP-11 and NLP-22 respectively (Turek et al., 2016; Nelson et 
al., 2013). These neuropeptides, conserved in fly and fish, engage GABA-ergic pro-sleep circuits (Meeusen et al., 2002; Lenz et al., 2015; Lee et al., 2017 ELife). The present conditioning paradigm involves repeated starvation followed by re-feeding. We have not identified the neuropeptides that control post-conditioning sleep, nor do we know which sleep circuit this paradigm may trigger. However, the circuit is likely to involve a stress-response component. The worms may grow exhausted due to liquid training, or become stressed by starvation. Another possibility is that worms naturally sleep periodically throughout the day and our training keeps them awake such that once they are returned to food, the entire population sleeps, perhaps due to a buildup of sleep pressure.

\section{What is it about the sleep brain state that predisposes memory consolidation?}

Changes in the brain state are indicative of sleep. Mammals cycle between rapid-eyemovement (REM) and non-rapid-eye-movement (NREM) sleep in which the neuronal activity in the thalamocortical and hippocampal systems alternates (Weber, 2017). Flies alternate between states of deeper and lighter sleep and these states are homeostatically-regulated as in mammalian sleep (van Alphen et al., 2013). Fly sleep has recently been revealed to have two stages of brain activity. One stage has increased oscillatory activity and the other has decreased activity, with the switch between the two brain states mediated by sleep and arousal-promoting neurons in the dFB, or the dorsal fanshaped body, in the central brain (Yap et al., 2017). The finding that flies go through a more active state in their sleep patterns means that when worms sleep, they too may have subtle head movements and other neuronal firing patterns. Skora et al., 2018 suggested that a head waving for example may indicate a state of reduced arousal.

C. elegans is no exception to having a brain state specific to sleep. Whole-brain calcium imaging has been reported on wake and sleeping $C$. elegans and this revealed that $40 \%$ of the worm's 300 neurons show oscillatory dynamics in the wake state that cycle through firing patterns indicative of different movement regimes (Nichols et al., 2017; Skora et al., 2018). 75\% of those neurons become inactive during lethargus. The notable exceptions are GABAergic and peptidergic head neurons, including the sleep-promoting interneuron RIS (Nichols et al., 2017), which have slow, stochastic cycles of calcium waves. In-depth analysis of each neuron's activity in the starvationinduced sleep state revealed that both of the paired AWC neurons are insensitive to sleep (Skora et al., 2018). Thus, AWC is potentially more active than AIY in the post-training animal. The picture that emerges from these observations is that the $C$. elegans brain is mostly quiet during sleep. 


\section{CREB and sleep-dependent memory consolidation}

Cyclic AMP response element binding protein, or CREB, is part of a highly-conserved process that has been shown to mediate long-term memory formation across phyla, including in flies, mollusks, mice (Silva et al., 1998), and more recently, in C. elegans appetitive learning (Lakhina et al., 2015). We found that $c r h-1(t z 2) / C R E B$ mutants become less responsive to butanone after 16 hours of recovery from conditioning-buffer-trained crh-1/CREB animals show a significantly lower chemotaxis index than wild-type animals (Figure S4). When we observed that crh1(tz2)/CREB mutants were able to acquire and retain aversive memory, we examined the olfactory phenotype and molecular nature of this often-used allele more closely.

The $c r h-1$ (tz2) allele we tested removes the last $60 \%$ of the highly-conserved basic leucine zipper (bZIP) domain, which mediates DNA binding and dimerization (Kimura et al., 2002). It has been previously reported that the $c r h-1(t z 2)$ mutant has irregular appetitive long-term memory in $C$. elegans (Lakina et al., 2015) and other abnormal behaviors, including clumping and burrowing in food-rich conditions (Kimura et al., 2002). It could be that for our aversive-learning paradigm, that the first $40 \%$ of the bZIP domain retains functionality. Conversely, the kinase inducible domain (KID) may be responsible for long-term negative-associative memory. The C. elegans KID contains the Ser 133 residue which is conserved in CREB family proteins in mammals and flies. This residue is recognized by the transcriptional co-activator CREB binding protein (CBP) and subsequently activate gene expression (Kimura et al., 2002; Lonze and Ginty, 2002). Perhaps, the KID domain is required for negative-associative memory and, by contrast, the bZIP domain is required for positive-associative memory. The two domains may mediate plasticity and memory via distinct downstream mechanisms. On the other hand, even though most forms of long-term memory require CREB, other transcription factors, including SFR, c-fos, EGR-1, and NF-K B, are also necessary for memory in flies and mammals (Kandel, 2012), and could contribute to $C$. elegans negative associative long-term memory.

\section{Aversive memory transitions from being independent of the AIY interneuron to depending on it}

We observed that neither AIB nor AIY (AWC presynaptic $\sim 5$ or $\sim 12$ synapses) was required for acquisition of the labile aversive memory of butanone, nor were they required for chemotaxis, but AIY is required for long-term memory. The finding that neither AIB nor AIY are required for acquisition, could indicate that another interneuron such as AIA is needed instead. AIA was shown to be required for memory induced by one training session with butanone and starvation (Cho et al., 2016). Memory in response to repeated, spaced-training may, likewise, require this interneuron for its acquisition. Our 
focus, however, is on long-term memory, thus, we examine cells such as AIB and AIY that are not required for either the primary response or acquisition of initial memory. The transition from short to long-term memory may reflect a sleep-dependent reorganization of the memory for long-term storage, perhaps being handled by mainly AIA to being dependent on both AIB and AIY neurons. In an interesting parallel, distinct brain regions, such as the mammalian hippocampus, the avian intermediate and medial mesopallium, and the insect antennal lobe handle newly acquired, labile memory and transfer this in a sleep-dependent process to ill-defined regions in the mammalian neocortex, an unidentified cortical region termed S' in birds (Jackson et al., 2008; Vorster and Born, 2015), and the mushroom body in insects (Hussaini et al., 2009) for long-term storage. Since discovering that worms sleep after training and that sleep is required for memory consolidation, it becomes possible to visualize the anatomically-compact nervous system of this transparent organism as memory is consolidated in real time. Thus, using C. elegans to understand the transition of memory may be a powerful way to elucidate the mechanisms by which long-term memories are formed.

The AIY interneurons regulate thermotaxis (Mori and Oshima, 1995), locomotion speed, and direction switch, and connect to downstream interneurons and motor command hubs via acetyl cholinergic connections to produce these behaviors (Li et al., 2014). In addition, AIY has been previously found to mediate plasticity to starvation paired with thermo- or chemotactic cues (de Bono and Maricq, 2005; Ishihara et al., 2002). AIY may function as a sensory integrator of information from upstream sensory neurons, including AWC in our paradigm. The butanone-sensing chemotaxis circuit is well characterized. Animals navigate up a gradient of butanone using a well-defined chemosensory circuit. The butanone-sensing AWC chemosensory neuron is silenced by the odor butanone and begins signaling upon butanone removal (Chalasani et al., 2007). Activation of AWC results in glutamate release onto AIY neurons that express glutamate-gated chloride channels (Chalasani et al., 2007). AIY neurons promote smooth, fast runs, and hyperpolarization of AIYs terminates these runs (Li et al., 2014). This results in a system in which animals sensing butanone have silenced AWCs and active AIYs, promoting the smooth, fast runs toward the butanone. When an animal moves away from the butanone, AWC is stimulated, and AIYs are prevented from stimulating runs. The AWCs also form excitatory synapses with AIB interneurons, which promote turning (Chalasani et al., 2007). Thus, when butanone levels are reduced and AWC becomes active, AIB interneurons promote pirouettes (Pierce-Shimomura et al., 1999). The synapses between AWC and AIY are inhibitory. Reduction of these synapses after butanone training and sleep could decouple AIY from the butanone-responsive AWC neuron, allowing it to promote forward locomotion, regardless of the 
butanone gradient. Thus, animals 16 hours post training would appear indifferent to the butanone gradient. However, other functions of AIY may not be affected, as they can still climb a benzaldehyde gradient, which is sensed by the non-butanone responsive AWC neuron as well (Wes and Bargmann, 2000). This mechanism of diminishing AWC-AIY synapses in a training and sleep-dependent manner could thus lead to the specific odor memory we observe.

\section{Inhibitory synapses and sleep}

The synapses between AWC and AIY are inhibitory through glutamate-gated chloride channels, which are only found in protostome invertebrate phyla (including mollusks, annelids, and arthropods). These glutamate-gated chloride channels mediate locomotion, feeding, and sensory input integration and closely resemble mammalian glycine receptors (Wolstenholme, 2012). Glycine receptors in the mammalian hippocampus also have an inhibitory role and may function in conjunction with excitatory NMDARs, or to inhibit GABAergic inhibition, to regulate hippocampal function, including learning and memory (Xu and Gong, 2010). In addition, $\alpha 2$-glycine receptor knockout mice show defects in spatial memory (Lin et al., 2017).

Not much is known about inhibitory synaptic dynamics. The inhibitory synapses of hippocampal neurons change in size considerably over time as compared to their excitatory counterparts, and their size configurations changed at much slower rates. Suppression of network activity only slightly affected remodeling dynamics, unlike in excitatory synapses (Rubinski and Ziv, 2015). Similar molecular processes may be at play in memory storage within the C. elegans olfactory circuit. It could be that these differing dynamics of inhibitory and excitatory synapses lead to homeostatic compensation of the system driven by sleep.

The SHY hypothesis says that the brain needs to reset during sleep to improve cellular health and to promote memory consolidation (Tononi and Cirelli, 2014). One mechanism for memory consolidation during sleep is synaptic homeostasis, where global synaptic strength decreases (Tononi and Cirelli, 2014). Though there is a preponderance of evidence for global synaptic downscaling, an upscaling of excitatory postsynaptic potentials was found to occur during slow-wave sleep (Chauvette et al., 2012). In addition, it was observed that synaptic downscaling in the hippocampus occurs instead during REM sleep (Grosmark et al., 2012). It was also observed that synaptic potentiation occurs in the mouse visual system during sleep after periods of visual experience, regardless of sleep cycle (Durkin and Aton, 2016). Surprisingly, neuronal firing rate homeostasis in mouse visual cortical neurons was suppressed by sleep and promoted by wake, and 
enhanced by a longer wake state (Hengen et al., 2016). Our paradigm may reveal a similar mechanism, where synapses are not taken down during sleep in the labile period immediately after training, but are instead decreased in awake animals 16 hours after spaced training.

The model posits that during wake, long-term potentiation (LTP) and long-term depression (LTD) modulate synaptic activity, and that during sleep, many synapses are downscaled potentially via a mechanism that differentially affects active and less-active synapses (Tononi and Cirelli, 2014). In our study, we observe significant synaptic reductions in both buffer- and butanone-trained animals during sleep. Interestingly, the two levels diverge later during subsequent wakefulness. We hypothesize that AWC-AIY synapses may be specified during butanone training and subsequent sleep, and the synaptic changes may be completed only after subsequent wakefulness. The training and sleep-dependent reduction in AWC-AIY synapses may be required to form the long-term odor memory. On the other hand, the reduced synapses may only be correlated with memory, but not causative. However, we find that AIY is required for long-term memory, and the observed reduction in AWC-AIY synapses would explain the long-term butanone indifference. Thus, we favor our hypothesis that sleep and training collaborate to produce long-term memory.

\section{Acknowledgements}

We would like to thank all of the L'Etoile, VanHoven, Kato, and Fang-Yen lab members for all of their helpful discussions, especially Katie Mellman, AJ Ablaza, Mary Futey, Sarah Woldemariam, Bo Zhang, Trang Duong, and Aarati Asundi. We would also like to thank Sara Alladin, Nebat Ali, Anjana Baradwaj, Claudia Echeverria, Jordan Mitchell, and Idan Siman-Tov for help with synapse experiments. We would like to thank Veronica Valdez for making the reagents to support the experiments in this manuscript. We are thankful to Andrei Goga, Maria Gallegos, Torsten Wittmann, Matt Gruner, Stephen Nurrish for all for all of their advice on experiments in this manuscript. We give a big thanks to the Caenorhabditis Genetics Center (CGC), supported by the NIH Office of Research Infrastructure Programs (P40 OD010440), for the strains they provided to us and for their support of the greater $C$. elegans community. We would also like to give another big thanks to the Sternberg lab and everyone else behind Wormbase.org (NIH grant \#U24 HG002223) and the Hall lab for Wormatlas.org (NIH grant \#OD010943 to DHH), both for their immensely helpful resources. Thanks to BioRender for their artwork which was used in Figure 1 and S1. This work was funded by the NIH, specifically R01DC005991 (N.L.D., M.K.V., C.F.Y.), R15NS109803 (M.K.V.), R01NS087544 (N.L.D., M.K.V.), R35GM124735 (S.K.), F31DC014921 (K.L.B.), and R01NS084835 (M.A.C.). 


\section{Author Contributions}

N.D.L., F.M.-L., K.L.B., M.K.V., S.K.N., C.B., F.F., A.B., A.V., J.L., E. C., K.A. and R.L.D. designed and conceived the experiments. F.M.-L., K.L.B., F.F., S.K.N., A.B., C.B., J.L., E.C., A.V., K.A., R.L.D., B.T., A.T., A. D. and N.D.L. performed the experiments. K.L.B., S.K.N., F.F., A.B., J.L., E.C. and A.V. analyzed the data. M.A.C. and C.F.Y. wrote code for and helped with the analysis. S.K. provided meaningful advice and co-mentoring and support of S.K.N. and R.L.D. N.D.L., K.L.B., M.K.V., F.M.-L., S.K.N., F.F., A.B., and A.V. wrote the manuscript.

\section{Declaration of Interests}

The authors declare no competing interests.

\section{Figure Titles and Legends}

\section{Figure 1. Spaced training induces long-lasting memory.}

(A) Schematic of aversive olfactory memory assay to induce long-lasting memory. Age-synchronized adult worms are rinsed from food and conditioned to either buffer (CTL, gray) or butanone (BTN, red) for 80 minutes followed by incubation with liquid food (OP50 of OD =10). After three cycles of conditioning, animals recover on NGM plates seeded with OP50 for either 0 hours or 16 hours before testing their attraction to BTN in a chemotaxis assay (1:1000 BTN dilution). Chemotaxis assay shown in Figure S1.

(B) Three-cycle training induces initial repulsion followed by long lasting memory 16 hours later. Aversive learning and memory in wild-type animals conditioned to either buffer (CTL, gray) or butanone (BTN, red) immediately post training ( $0 \mathrm{hr}$ ) or after 16 hours of recovery on food (16 hr). The Chemotaxis Index $(\mathrm{Cl})$ is calculated and is the number of worms on the control subtracted from the number on butanone, divided by the total number of worms, not including worms at the origin. $A$ positive chemotaxis index $(\mathrm{Cl})$ indicates attraction and negative, aversion. $\mathrm{N}=$ number of trials where all trials are done on independent days and each grey dot represents an individual assay day, with 50-200 animals/day. Error bars represent S.E.M. Statistical significance was reported as ${ }^{* * *} P<0.001$, ${ }^{* *} P<0.01$, ${ }^{*} P<0.05$, and NS is $P>0.05$. The Shapiro-Wilk normality test was performed to determine data distribution, followed by the Kruskal-Wallis test, an analysis of variance of multiple comparisons for non-parametric data $\left.{ }^{* * *} P<0.001\right)$. If normally distributed, then $P$ values were generated with $a$ Student's unpaired t-test. If non-parametric, then $P$ values were generated with the Mann-Whitney utest. Hochberg adjustment for multiple comparisons was performed on all P-values generated from data included in the same graph to control Type I error. The t-test was performed on CTL $0 \mathrm{hr}$ vs CTL 
$16 \mathrm{hr}$ and CTL $16 \mathrm{hr}$ vs BTN $16 \mathrm{hr}$. The u-test was performed on CTL $0 \mathrm{hr}$ vs BTN $0 \mathrm{hr}$ and BTN $0 \mathrm{hr}$

vs BTN $16 \mathrm{hr}$. Behavioral data throughout the paper are represented in this same way with the same numbers of animals on independent days. Additionally, throughout the paper, the same statistical analysis was performed on the data unless otherwise noted in the figure legend.

(C) Three cycle butanone training does not affect attraction to benzaldehyde after 16 hours of recovery. Chemotaxis indices of animals trained to buffer (CTL, gray bars, gray dots) or butanone (BTN, red bars, gray dots) and tested for attraction to butanone or of animals trained to buffer (CTL, gray bars, white dots) or benzaldehyde (BENZ, red bars, white dots) and tested to benzaldehyde. The t-test was performed on CTL vs BTN both tested on BTN at $16 \mathrm{hr}, \mathrm{CTL}$ vs BTN both tested on BENZ at $16 \mathrm{hr}$, and BTN $16 \mathrm{hr}$ tested to BTN vs BTN $16 \mathrm{hr}$ tested to BENZ. The u-test was performed on CTL vs BTN $0 \mathrm{hr}$ both tested on BTN, BTN $0 \mathrm{hr}$ tested on BTN vs BTN $0 \mathrm{hr}$ tested on BENZ, CTL vs BTN Ohr both tested on BENZ, and BTN $0 \mathrm{hr}$ test on BENZ vs BTN $16 \mathrm{hr}$ tested on BENZ. For every graph in this paper, the Kruskal-Wallis test was performed and yielded ${ }^{* * *} P<0.001$, unless otherwise noted.

See also Figures S1, S2, S3, and S4.

\section{Figure 2. Memory is labile immediately post training.}

(A) Chemotaxis indices of animals conditioned to CTL or BTN. After three cycles of conditioning, animals recover on NGM plates seeded with OP50 for either 0 minutes, 15-30 minutes, 45-60 minutes, 75-90 minutes, 120 minutes, or 16 hours before testing their attraction to BTN in the chemotaxis assay. The $P$ value is ${ }^{* * *} P<0.001$ for all of the comparisons between the CTL and BTN of the same recovery time. The t-test was performed on CTL vs BTN 15-30', BTN 45-60' vs BTN 75-90', BTN 75-90' vs BTN 120', and BTN 120' vs BTN $16 \mathrm{hr}$. The u-test was performed on CTL vs BTN $0 \mathrm{hr}$, CTL vs BTN 45-60', CTL vs BTN 75-90', CTL vs BTN 120', CTL vs BTN 16 hr, BTN 0 hr vs BTN 1530', BTN 15-30' vs BTN 45-60', BTN 0 hr vs BTN 120', BTN 15-30' vs BTN 120', and BTN Ohr vs BTN $16 \mathrm{hr}$.

(B) Learning indices of spaced-trained animals. The learning index is calculated as the chemotaxis index of the BTN animals subtracted from the chemotaxis index of the CTL animals. The higher the learning index, the more the animals have learned and thus kept the BTN memory. The t-test was performed on 0 hr vs 15-30', 15-30' vs 45-60', 120' vs 16 hr, 0 hr vs 120', 15-30' vs 120', and 0 hr vs $16 \mathrm{hr}$. The u-test was performed on 45-60' vs 75-90' and 75-90' vs $120^{\prime}$. 


\section{Figure 3. C. elegans sleeps after spaced-training.}

(A) Untrained animals are more active than butanone spaced-trained animals during the 90 minutes after training. Raster plot of activity of untrained (left panel) vs spaced-trained animals to BTN (right panel). Yellow indicates activity and dark blue, quiescence. Individual animals were placed into a PDMS 48-well microtiter plate (WorMotel, Churgin et al. 2017) and their movement was recorded by camera. Movement was analyzed with a custom MatLab script. Each row represents an individual worm's movement over 90 minutes. Quiescence was defined as no movement for $>30$ seconds and marked with a blue raster.

(B) Untrained animals are less quiescent than BTN animals, and CTL or BTN have no difference in quiescence. Mean total quiescence in total minutes per hour of animals in WorMotel that are untrained (black bars, grey dots) or CTL or BTN animals. Each grey dot represents 24 animals tested per condition on an independent day, and this is the same for every mean total quiescence graph in this paper. The t-test was performed on untrained vs BTN (first and second bars) and the u-test was performed on CTL vs BTN (third and fourth bars).

(C) Feeding rate is reduced post odor training. Feeding rate is measured as pharyngeal pumps per minute. Each dot represents one animal. Assay was performed on three separate days with animals that were untrained (black bar, grey dots) vs BTN. Pharyngeal pumping was scored manually. Error bars represent S.D. The u-test was performed.

(D) Butanone-trained animals have an arousal delay in compared to untrained animals. Movement response latency was measured as number of seconds until a sinusoidal wave is initiated (an escape response) after exposure to blue LED light and 1.2 KHz of vibrations in untrained (black bar, grey dots) or BTN animals. Videos were taken of animals over three days. The u-test was performed. (E) Butanone-trained animals are less active upon an arousal stimulus than untrained animals. Activity post stimulus was measured by counting how many full sinusoidal wave movements were completed in 30 seconds after exposure to blue LED light and $1.2 \mathrm{KHz}$ vibrations stimuli. Videos were taken of animals over three days. The u-test was performed.

\section{Figure 4. Sleep is required post training for long-term memory.}

(A). Animals are less quiescent off food than on food in one hour post training. Mean total quiescence was measured in minutes per hour of animals after training either on food (first bar) or off food (second bar). Individual animals were placed into a PDMS 48-well microtiter plate (WorMotel, Churgin et al. 2017) either seeded with food (OP50 in 24 of the wells for the "on food" condition) or without food ("off food"), and animals were recorded with a camera. Movement was analyzed with a custom MatLab script. The u-test was performed. 
(B) Animals off food for two hours immediately after training do not maintain the memory 16 hours later. After three cycles of conditioning, animals are immediately tested in a chemotaxis assay or recovered on either unseeded NGM plates or NGM plates seeded with OP50 for 120 minutes before testing 16 hours after the end of the training assay. Chemotaxis indices of animals conditioned to buffer (CTL, gray) or butanone (BTN, red), tested after $0 \mathrm{hr}$ or $16 \mathrm{hr}$ post training, or animals off food for $2 \mathrm{hr}$ and on food for $14 \mathrm{hr}$ (CTL, lighter gray with darker gray outline, fifth bar, or BTN, lighter red with darker red outline, sixth bar). The t-test was performed on CTL $16 \mathrm{hr}$ vs BTN $16 \mathrm{hr}$, CTL $16 \mathrm{hr}$ vs CTL 2 hr off, $14 \mathrm{hr}$ on food, BTN $16 \mathrm{hr}$ vs BTN 2 hr off, $14 \mathrm{hr}$ on food, and CTL 2 hr off, 14 hr on food vs BTN $2 \mathrm{hr}$ off, $14 \mathrm{hr}$ on food. The u-test was performed on CTL $0 \mathrm{hr}$ vs BTN Ohr.

(C) Animals overexpressing the neuropeptide flp-11 are more quiescent than control animals. Mean total quiescence was measured in minutes per hour. Hsp16.2::flp-11 ("pHS::flp-11") animals overexpress the flp-11 neuropeptide after heat-shocking. The heat shock was performed after spaced training for $5 \mathrm{~min}$ at $37^{\circ} \mathrm{C}$. The $p H S$ ::flp-11 off food animals (first bar, pink outline) were off food for one hour while filmed in the WorMotel and the pHS::flp-11 off food 5' HS animals (second bar, purple outline) were heat-shocked for five minutes off food and then put into the WorMotel without food and filmed for one hour. The t-test was performed.

(D) Animals that overexpress flp-11 via heat-shock keep the memory off food better than heatshocked wild-type animals off food. Chemotaxis indices measured immediately after training (0 hr) for wild-type animals conditioned to CTL (first bar), BTN (second bar), and of BTN-trained animals expressing the pHS::flp-11 transgene (purple, third bar), and trained animals heat-shocked for $5^{\prime}$ at $37^{\circ} \mathrm{C}$, two hours off, 14 hours on food ("2 hr off $14 \mathrm{hr}$ on food") including CTL (lighter gray, fourth bar), BTN (lighter red, fifth bar), and pHS::flp-11 (lighter purple with dark purple outline, sixth bar). Heatshocked pHS::flp-11 animals were confirmed to all be quiescent after the heat pulse. The t-test was performed on CTL 0 hr vs pHS::flp-110 hr, CTL 5' HS + 2 hr off, 14 hr on food vs BTN 5' HS + 2 hr off, 14 hr on food, BTN 5' HS + 2 hr off, 14 hr on food vs pHS::flp-11 5' HS + 2 hr off, 14 hr on food, and CTL 5' HS +2 hr off, 14 hr on food vs pHS::flp-11 5' HS + 2 hr off, 14 hr on food. The u-test was performed on CTL $0 \mathrm{hr}$ vs BTN $0 \mathrm{hr}$ and BTN $0 \mathrm{hr}$ vs pHS::flp-11 $0 \mathrm{hr}$.

See also Figure S5.

Figure 5. AWC sensory activity is slightly altered only immediately after spaced-training. Calcium responses in AWC ${ }^{O N}$ for CTL (blue) or BTN (red) animals to removal $(A, C)$ or addition (B, D) of butanone measured immediately after training (A-B) or after $16 \mathrm{hr}$ of recovery (C-D). Video recordings were taken for 60 seconds and the percent fluorescence intensity of the GCaMP3 
fluorophore was measured and graphed as $\Delta F / F_{0}$ for each animal tested. The worms are immobilized in a PDMS microfluidic chip and the gray shading indicates butanone being flowed over the worm nose via laminar flow for 30 seconds and no shading means that buffer was being flowed. Each line indicates a recording from one worm and the bold lines indicate the means. The $\mathrm{Cl}$ of the populations that these animals came from showed that the CTL animals were attracted to butanone $($ mean $\mathrm{Cl}=$ 0.9 ) and each population of BTN animals was repulsed (mean $\mathrm{Cl}$ at $0 \mathrm{hr}$ recovery $=-0.01$, mean $\mathrm{Cl}$ for $16 \mathrm{hr}$ recovery $=-0.57$ ). The scatter plot panels on the right of each calcium recording panel contain the absolute values of the deltas between the mean $\Delta F / F_{0}(\%)$ values from 10 seconds before stimulus on or offset and the mean $\Delta \mathrm{F} / \mathrm{F}_{0}(\%)$ values 10 seconds after stimulus on or offset, where each dot signifies one worm. The black bars indicate the means. The t-test was performed on all of the comparisons.

\section{Figure 6. Memory acquisition requires AIB or AIY and long-lasting memory requires} AIY.

(A) AWC olfactory circuit of C. elegans. Sensory neuron AWC (red) is inhibited by odor. The AIY (yellow) interneuron promotes straight runs and is inhibited by AWC while the AIB (blue) interneuron promotes turns and is activated by AWC. Thus, odor activates AIY and inhibits AIB allowing the animal to run up an odor gradient and reorient if going down the gradient. Smallest arrow indicates 110 synapses between the two neurons, medium arrow, 10-100 synapses, and largest arrow, more than 100 synapses. Only chemical synapses are indicated in the circuit (gap junctions not shown). Figure adapted from Gordus et al., 2015.

(B) Killing AIB and AIY somewhat inhibits memory acquisition and killing AIY blocks memory maintenance. Chemotaxis indices after 0 or $16 \mathrm{hr}$ of recovery of wild-type vs animals carrying a transgene or mutation to kill specific interneurons including AIB (blue), AIY (yellow) or AIY and AIB (green). The t-test was performed on CTL vs BTN $0 \mathrm{hr}$, CTL vs BTN AIB- $0 \mathrm{hr}$, CTL vs BTN AIY- 0 hr, CTL vs BTN AIB-, AIY- 0 hr, BTN vs BTN AIB- 0 hr, BTN AIB- vs BTN AIY- 0 hr, BTN AIY- vs BTN AIB-, AIY- 0 hr, BTN vs BTN AIY- 0 hr, BTN vs BTN AIB-, AIY- 0 hr, BTN AIB- vs BTN AIB-, AIY- 0 hr, CTL vs BTN AIB- 16 hr, CTL vs BTN AIY- 16 hr, CTL vs BTN AIB-, AIY- 16 hr, and BTN 16 hr vs BTN AIY- $16 \mathrm{hr}$. The u-test was performed on CTL vs BTN AIB- $16 \mathrm{hr}$, BTN $16 \mathrm{hr}$ vs AIB- $16 \mathrm{hr}$, BTN AIBvs BTN AIY- 16 hr, BTN AIY- vs BTN AIB-, AIY- 16 hr, BTN AIB- vs BTN AIB-, AIY- 16 hr, and BTN 16 hr vs BTN AIB-, AIY- 16 hr. 


\section{Figure 7. AWC-AIY synapses are diminished in butanone-trained animals allowed to sleep} directly after training.

(A) Schematic of split GFP-based AWC-AIY NLG-1 GRASP marker. Circles represent cross-sections of the AWC and AIY neurites, and one of each neurite is represented for simplicity. Fragments of the split GFP are linked to the pre- and postsynaptically localized protein NLG-1 (Neuroligin 1), and expressed in the AWC and AIY neurons with the selective promoters odr-1 and ttx-3. When synapses form between the neurons, the split GFPs reconstitute and fluoresce. Small white circles indicate a presynaptic site, and crosshatching represents a postsynaptic site.

(B) Schematic of the head of an animal in which NLG-1 GRASP labels synapses between the AWC (red) and AIY (beige) neurites.

(C) Schematic and micrographs of a buffer-trained animal carrying the AWC-AIY NLG-1 GRASP marker with the AWC neuron labeled in red with the cytosolic mCherry fluorophore. Synaptic fluorescence is observed in a punctate pattern spread across the arc of the AWC axons. The area in the gray box is expanded in the rightmost image.

(D) Micrographs of AWC-AIY NLG-1 GRASP fluorescence in animals trained with butanone (BTN) or control buffer (CTL), placed on or off food during the first two hours after training, and recovered on food for an additional 14 hours, as described in Figure 4. AWC-AIY NLG-1 GRASP fluorescence is reduced in the animal trained with butanone and recovered on food for the first 2 hours post-training. (E) Quantification of the reduction in AWC-AIY NLG-1 GRASP fluorescence intensity in animals trained with butanone (BTN) and recovered on food for the first two hours post training in comparison to animals trained with a control buffer (CTL), or animals trained with butanone and recovered off food for the first two hours post training. N>90 for each box and includes animals trained on four different days. ${ }^{* *} \mathrm{P}<0.001$, NS $\mathrm{P}>0.05$, Mann-Whitney u-test. $\mathrm{P}$ values were adjusted for multiple comparisons using the Hochberg procedure. (F) Quantification of AWC-AIY NLG-1 GRASP fluorescence intensity at 0 hours, 2 hours and 16 hours post-training in buffer-trained (CTL) and butanone-trained (BTN) animals allowed to sleep for the two hours post-training. N>75 for each box and includes animals trained on four different days. ${ }^{* *} \mathrm{P}<0.001,{ }^{* *} \mathrm{P}<0.01, \mathrm{NS} \mathrm{P}>0.05$, MannWhitney u-test. $\mathrm{P}$ values were adjusted for multiple comparisons using the Hochberg procedure.

See also Figures S6 and S7. 


\section{STAR Methods}

\section{CONTACT FOR REAGENT AND RESOURCE SHARING}

Further information and requests for resources and reagents should be directed to and will be fulfilled by the Lead Contact, Noelle L'Etoile (noelle.letoile@ucsf.edu)

\section{EXPERIMENTAL MODEL AND SUBJECT DETAILS}

\section{C. elegans strain cultivation}

All C. elegans worms were reared according to standard protocols (Stiernagle, 2006). All strains were raised and tested at $20^{\circ} \mathrm{C}$. Animals were raised on $10 \mathrm{~cm} \mathrm{NGM}$ plates seeded with OP50 E. coli. All assays were conducted with one-day old adult worms, unless noted as two day-old adults in the figure legends. All strains used in this study are listed in the Key Resources Table. The JZ2008 AIB and AlY double kill strain (ttx-3(ks5) X; pels578(pnpr-9::casp1; punc-122::mCherry; pnpr-9::venus) was made by mating the FK134/ttx-3(ks5) X strain with the ; pels578(pnpr-9::casp1; punc-122::mCherry; pnpr-9::venus) strain from the CGC and the lino lab, respectively. Other transgenic strains generated for this study are iyls35 (pttx-3::nlg-1::GFP1-10 (Feinberg et al., 2008) (70ng/Nl), podr-1::nlg-1::GFP11

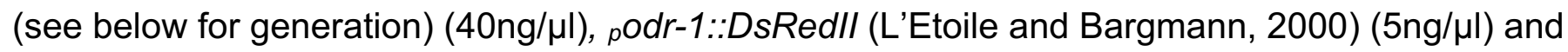
punc-122::RFP (Loria et al., 2004) (20ng/ul)) and wyls155 (pgpa-6::nlg-1::GFP1-10 (Park et al., 2011)

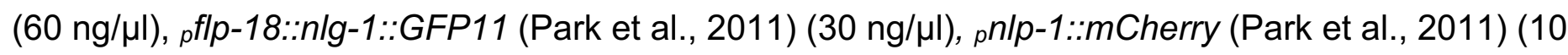

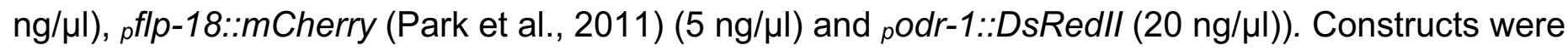
generated using standard molecular techniques. To generate $p$ odr-1::nlg-1::GFP11, the odr-1 promoter was amplified from ${ }_{p}$ Odr-1::DsRedII (L'Etoile and Bargmann, 2000) using ${ }_{p}$ odr-1-specific primers (MVP578: TTGCATGCCTGCAGGTCG, which has an internal Sphl site and MVP581: GACTGGCGCGCCTACCTTTGGGTCCTTTGGC, which introduces an Ascl site). Then, the ${ }_{p}$ odr-1 fragment was subcloned into the Sphl-Ascl fragment from nlg-1::GFP11 (Park et al., 2011).

\section{METHOD DETAILS}

\section{Chemotaxis assay}

To prep worms for behavior, we picked 4-5 larval stage 4 (L4) animals onto $10 \mathrm{~cm}$ NGM plates seeded with OP50 E. coli and grew them up for 5 days at $20^{\circ} \mathrm{C}$ when worms were one-day old adults. It is critical that the strains be completely clean with no fungal and bacterial contamination of any kind. 
For single-cycle odor training (performed in Figure S2), the adult worms were washed with S. basal buffer $\left(0.1 \mathrm{M} \mathrm{NaCl}, 0.05 \mathrm{M} \mathrm{K}_{3} \mathrm{PO}_{4}, \mathrm{pH} 6.0\right)$ off of $10 \mathrm{~cm}$ NGM plates and into tubes, where they were washed three times with S. basal buffer. The animal population was split in half where one half was soaked in S. basal and the other half was soaked in a 1:10,000 dilution of butanone or diacetyl in S. basal buffer. Animals were rotated in tubes on a rotisserie for 80 minutes, where they were then washed an additional three times in S. basal.

Chemotaxis plates were made as following: for $100 \mathrm{~mL}$ of media, we added $100 \mathrm{~mL} \mathrm{ddH}_{2} \mathrm{O}$ into a flask with $1.6 \mathrm{~g}$ of Difco agar, agar agar, or bacto agar, boiled it in the microwave until dissolved, added a magnetic stir bar and stirred until cooled to $50-55^{\circ} \mathrm{C}$, then added $500 \mathrm{uL}$ of $1 \mathrm{M}$ $\mathrm{K}_{3} \mathrm{PO}_{4}, 100 \mathrm{uL} 1 \mathrm{M} \mathrm{CaCl}_{2}$ and $100 \mathrm{uL} 1 \mathrm{M} \mathrm{MgSO}_{4}$. We Pipetted $10 \mathrm{~mL}$ of media into $10 \mathrm{~cm}$ plastic petri dishes and let it cool to solidify. We drew assay plate guides as shown in Figure S1. We put 1 uL of (M) $\mathrm{NaN}_{3}$ onto the middle of the odor and diluent arenas, let it dry, and then added $1 \mathrm{uL}$ of 200 proof ethanol to the diluent (control) arena and for the odor point sources, added $1 \mathrm{uL}: 1000 \mathrm{uL}$ butanone to ethanol, or $1 \mathrm{uL}: 1000 \mathrm{uL}$ diacetyl to ethanol, or $1 \mathrm{uL}: 200 \mathrm{uL}$ benzaldehyde to ethanol, to the odor arena. When plating animals after three cycles of training, we did two washes with S. basal and then a third wash with $\mathrm{ddH}_{2} \mathrm{O}$ (to prevent worms from staying at the origin of the chemotaxis plate, since they are attracted to the salt in S. basal). We plated animals at the origin of the plate (bottom) and wicked away any excess moisture with a Kim Wipe, being careful not to cause any gouges in the agar to prevent burrowing. We put 50-400 worms on the origin and wicked up any moisture, and then let them roam at least 2 hours and calculated chemotaxis indices.

To calculate a chemotaxis index, after at least two hours of roaming on the plate, we counted how many worms were within the odor arena, subtracted from that number the number of worms at the diluent (200 proof ethanol) arena, and then divided that by the total number of worms on the plate that were not at the origin.

\section{LTM chemotaxis assay}

The single-worm odor training protocol from above was used, but there are three $\times$ 80-minute buffer or odor treatment cycles with two x 30-minute bacteria recovery periods in between. After the first 80-minute training cycle, the bacteria recovery period was performed. First, to make the concentrated bacteria, $100 \mathrm{~mL}$ of LB was seeded with OP50 and was shaken overnight at $37^{\circ} \mathrm{C}$ at 250 RPM until it reached an OD of 10 and then centrifuged at 4000 RPM for 15-30 minutes and then the pellet(s) was resuspended in 37-38 mL of S. basal buffer. Next, $1000 \mathrm{uL}$ of concentrated OP50 
was added to the tubes and rotated for 30 minutes, which completes one cycle of recovery post one cycle of buffer or odor treatment. We repeated the buffer or odor treatment cycle, followed by one more cycle of recovery with OP50, then a third treatment cycle, and then washed and put worms on chemotaxis plates as described under the chemotaxis plates protocol above.

For overnight recovery animals, we washed part of the population onto $5.5 \mathrm{~cm}$ OP50-seeded NGM plates and kept them at $20^{\circ} \mathrm{C}$ for 16 hours, before washing three times and plating. For recovery periods different from 16 hours, we washed them onto seeded (or unseeded for the sleep assay) plates for whatever time was desired (e.g. 30 minutes or 2 hours).

\section{LTM NLG-1 GRASP assays}

The training paradigm described above was modified to accommodate NLG-1 GRASP imaging. Approximately 30 plates of worms were prepared to allow enough worms for multiple batches and imaging. Worms were divided into four batches that began training 40 minutes apart. Batches 1 and 3 were given the buffer treatment, while batches 2 and 4 were trained in the odor solution.

LTM NLG-1 GRASP 16-hour assay: After three cycles of training (described above), half the worms from each batch were placed on seeded plates, and half were placed on unseeded plates, then transferred to seeded plates after two hours. All plates were incubated at $20^{\circ} \mathrm{C}$ until 16 hours post-training, when worms were washed (as described above). 20 worms from each of the 8 batches were anesthetized and imaged using a Zeiss Axio Imager.A1 compound fluorescent microscope and Axiovision software (see "Synapse Imaging and Analysis" below), and 50-400 worms from each of the 8 batches underwent the butanone chemotaxis assay.

LTM NLG-1 GRASP 0-hour, 2-hour, and 16-hour assay: After three cycles of training, worms from batches 1-4 were each divided into three groups so that imaging and chemotaxis experiments could be performed at three timepoints: 0 hours post-training, 2-hours post-training, and 16-hours post-training.

0-Hour Imaging and Chemotaxis: After training, worms from all four batches were washed (as described above). From each batch, 20 worms were separated, anesthetized, and imaged under the Zeiss Axio Imager.A1 compound fluorescent microscope and Axiovision software (see "Synapse Imaging and Analysis" below), and 50 to 400 worms were assessed for butanone chemotaxis. 
Two-Hour Imaging and Chemotaxis: After training, worms from batches one and two were each placed on seeded NGM plates. After two hours, animals were washed as described above. 20 animals from each of these batches were anesthetized and imaged (see "Synapse Imaging and Analysis" below), and 50 to 400 worms from each of these batches were assessed for butanone chemotaxis.

16-Hour Imaging and Chemotaxis: After training, worms from batches one, two, three, and four were each placed on seeded NGM plates. After 16 hours, animals were washed as described above. 20 animals from each of these batches were anesthetized and imaged (see "Synapse Imaging and Analysis" below), and 50 to 400 worms from each of these batches were assessed for butanone chemotaxis.

\section{WorMotel making and analysis}

To make the WorMotel (Churgin et al., 2017), we used a PDMS chip with 48 total wells made according to the paper or the online resource (http://fangyenlab.seas.upenn.edu/links.html). Next, we made $100 \mathrm{~mL}$ NGM by adding together $2.1 \mathrm{~g}$ low melting-point agarose, $0.3 \mathrm{~g} \mathrm{NaCl}, 0.25 \mathrm{~g}$ bacto peptone, and $3 \mathrm{uL}$ tween 20 (to keep a flat agar surface). We boiled the media in the microwave, cooled it down to $\sim 50-58^{\circ} \mathrm{C}$, then added $100 \mathrm{uL} \mathrm{CaCl}$, $100 \mathrm{uL}$ cholesterol dissolved in $\mathrm{EtOH}, 100 \mathrm{ul}$ $\mathrm{MgSO}_{4}$, and $2.5 \mathrm{~mL} \mathrm{~K}_{3} \mathrm{PO}_{4}$. We next added $10 \mathrm{uL} /$ well of chip, putting the pipet tip inside the well to avoid bubbles. We did this fast because the chip may dry and crack. About 10-30 minutes after adding the agarose, we added 5 uL of OP50 (same as the concentrated bacteria used for recovery in the training paradigm). It takes about 1-2 hours for the bacteria to dry at room temp, depending on the humidity. We made a humidity chamber for the WorMotel by adding $0.1 \mathrm{~g}$ of gel soil (we used Soil Moist granules) with $15 \mathrm{~mL}$ water to a $10 \mathrm{~cm}$ plastic Petri dish (it takes about 30 min until the soil is properly hydrated). You can re-use the humidity chamber by adding the correct amount of water to come back up to the right weight as before.

We picked one worm per well onto the WorMotel. We used the left $4 \times 6$ wells loaded with our control worms and the right $4 \times 6$ wells loaded with our experimental group for ease of analysis. We waited $15 \mathrm{~min}$ after picking worms on to the WorMotel for them to settle. The lid was kept on the chamber whenever possible to prevent the agarose from cracking to prevent worms from burrowing. Fogging often occurs, so we would rub either Tween 20 or anti-fog lens cleaner for eyeglasses (we like Cat Crap brand) on the dish lid before imaging. 
The camera we used to acquire images was a Teledyne Dalsa PT-21-04M30 Camera Link Camera (Dalsa Proprietary Sensor 2352×1728 Resolution Area Scan Camera) attached with a Linos Rodagon Modular-Focus lens $(f=60 \mathrm{~mm})$ attached. We used a four-piece metal post imaging stand with a plastic stage, but any imaging stand will do as long as the imaging distance allows you to see the whole WorMotel with the camera. We used a red LED light stand to illuminate all around the WorMotel for imaging (we specifically used 2-inch long $4 \times 6$ LED strips and 5-inch long $4 \times 12$ LED strips (from www.Oznium.com) in a two-concentric square formation. We used a T175 tissue culture flask filled entirely with water added to as the cooling chamber as a buffer against the heat generated by the red LED lights. We put the flask on top of LED stand. We had an imaging setup closed off from any light interruptions (we used black curtains).

We used the Multiple-Worm Tracker (MWT 1.3.0r1041) made by Rex Kerr. Hit "use selected import source," go to "Raw Image" in pull-down menu, change "experiment duration" to 3600 seconds (1hr) or whatever time is desired. We would make a new folder in icon below "experiment duration" and name files in box below (e.g. name and date). Then, we put 3 as "raw image save interval" (1 image every $3 \mathrm{sec}$ ). Next, you would hit the green "Go" button. PNG files were then generated.

We renumbered the PNG files (we used Irfanview for Windows developed by Irfan Škiljan to rename our files "image\#\#\#" to use with our Matlab script). We analyzed images with Matlab script "KS_analysis_CFY_Jan2019.m” and used the GUI “MC_QuiescenceActivity_v1202.fig," which are available online at https://github.com/cfangyen/LEtoile_WorMotel.

Once images were renumbered, we used Matlab and typed "guide" into the command window to launch the GUI "MC_QuiescenceActivity_v1202.fig" to open GUI (to segment images into one file). Next, we pressed the "play" button and then pressed "load images" and then "process images." We opened the first image (e.g. "image0001") into the GUI. We followed the GUI listed instructions, which tells you to click in the middle of the well/room on the top left, then the top right, then the bottom left, and then the bottom right (in a " $Z$ " pattern) to define the motel size and position. Then, clicked on one representative well at the top left and the top right to define the well/room sizes. We edited the parameters by putting image 1 to the last number minus 1 (e.g. if 1181 images, we had it go from 1 to 1180). We set the threshold (we used about 0.25-0.3, but check if it looks good (should see worms marked in white with not too much background white noise). We then saved the file and then ran it.

Once finished processing, we saved the file as the same file name we created before running it and replaced it with the new one (if desired to avoid confusion, but you can also keep them as 
separate files). We then opened the script "KS_analysis_CFY_Jan2019.m" in Matlab. We clicked in the first cell and ran it and then opened the processed type file we just created. Next, we clicked in the second cell and ran it to run the analysis and produce the raster map figures. The output gives you the KS analysis graphs for total quiescence on top and the longest quiescence graph on the bottom, with the KS test $\mathrm{P}$ value reported at the top of each graph. It will also output Excel files with Group 1 being the control worm quiescence (in minutes) values (assuming your control group was loaded to the left $4 \times 6$ wells), with the left column as total quiescence and the right column being longest quiescence bout (in minutes) and Group 2 being the experimental group (assuming the experimental group was loaded to the right $4 \times 6$ wells). The Group 1 quiescence map is on top and the Group 2 (experimental group) map is on the bottom, where blue marks quiescence over time and yellow marks movement (movement in 30 seconds). You will also get the blue heatmaps and the yellow and blue raster plots (without the graphs in the same figures) as output. We saved these as .fig files to open in Matlab and also as .png files to open it with any picture preview program

\section{Pharyngeal Pumping Assay}

This assay was performed by standard methods (Raizen et al., 2012). To perform the assay, we watched the pharynx of a worm under a stereomicroscope at 40-50X magnification and once the grinder in the terminal bulb does one complete contraction and relaxation, or "pump," we counted that as one pump, using a counter to count every time they complete a full pump for 10 seconds. Then, we disposed of the worm to prevent re-counting of the same animal. We took the number of pumps completed and multiplied that by 6 to find the pharyngeal pumping rate in pumps per minute.

\section{Quiescence disruption}

We assessed animal responsiveness to a stimulus which has been previously shown to disrupt quiescence in C. elegans (Nagy et al., 2014). Specifically, 3.5 or $5.5 \mathrm{~cm}$ NGM plates seeded with OP50 with 20-30 worms were placed on a $50 \mathrm{~mm}$ piezo $1.2 \mathrm{KHZ}$ piezo buzzer elements (Digikey \#668$1190-N D)$. Piezo elements were supplied with $5 \mathrm{~V}$ with a $50 \%$ pulse-width modulated duty cycle using an Arduino-style microcontroller and its accompanying software, using the code named "Arduino_blink_buzz" accessible at www.GitHub.com/letoilelab. Stimulus onset was synchronized with video recording by flashing a blue LED, used at the maximum light intensity (we used Digikey \#1528-2334-ND) at a distance of about $10 \mathrm{~cm}$ during video recording. In cases where animals were exposed to prolonged stimulation, animals were subjected to blocks of stimulation for 5 minutes with the blue light flashing for 1 second every 20 seconds, followed by no stimulation for 5 minutes. Videos 
were recorded on an Imaging Source DMK 23GP031 camera using Micromanager software (Edelstein et al., 2014).

\section{Heat-shock assay}

C. elegans animals were heat shocked at $37^{\circ} \mathrm{C}$ for 5 minutes in a water bath while on $5.5 \mathrm{~cm}$ unseeded NGM plates covered in parafilm. After the heat shock, animals were put at $20^{\circ} \mathrm{C}$ until behavior was assessed by the chemotaxis assay.

\section{Calcium imaging}

Calcium imaging of the AWC ${ }^{O N}$ neuron was performed on lines expressing the geneticallyencoded calcium indicator GCaMP3 (Tian et al., 2009) under the str-2 promoter (JZ1795/pyls701 (pstr-2::GCaMP3; pofm-1::GFP; pceh-36::mCherry)). One-day-old adult worms were conditioned to either buffer or $1.23 \mathrm{mM}$ butanone diluted in S-basal buffer (the same concentration as used for the butanone conditioning mentioned in "Chemotaxis assay") during three, 80-minute training cycles (interspersed) with feeding (described in "LTM chemotaxis assay"). Immediately after the end of the third training cycle or after a 16-hour overnight recovery on food, worms were rinsed three times in S-basal buffer and loaded into a custom, polymer polydimethylsiloxane (PDMS) microfluidic device (Chronis et al., 2007). The nose of the animal was exposed to liquid streams of either S-basal buffer or $1.23 \mathrm{mM}$ butanone. A manual switch attached to a solenoid valve was used to direct the buffer or odor stream across the nose of the worm. The stimulation protocol consisted of exposing worms to S-basal buffer for 30 seconds followed by a 30 second exposure to $1.23 \mathrm{mM}$ butanone (odor on) or by exposing worms to $1.23 \mathrm{mM}$ butanone for 30 seconds followed by a 30 second exposure to S-basal buffer (odor off). Fluorescence was monitored with a Zeiss 40X air objective on an inverted microscope (Zeiss Axiovert 200). Images were taken at rate of $2 \mathrm{~Hz}$ with a blue light exposure time of $100 \mathrm{~ms}$ using an ORCA-Flash 2.8 camera (Hamamatsu).

\section{Synapse imaging}

A Zeiss Axio Imager.A1 compound fluorescent microscope (Figures 7E and F, and Figure S6) and a Zeiss LSM710 confocal microscope (Figure 7C and D) were used to capture images of live $C$. elegans under 630X magnification. Worms were anesthetized on $2 \%$ agarose pads using a 2:1 ratio of 0.3 M 2,3-butanedione monoxime (BDM) and $10 \mathrm{mM}$ levamisole in M9 buffer. All micrographs taken were of one-day old and two-day old gravid adults. 


\section{QUANTIFICATION AND STATISTICAL ANALYSIS}

\section{Behavioral analysis}

Statistics were performed using Graphpad Prism 8 and RStudio. P values are used for the statistical readouts, with the following notations: NS is $P>0.05,{ }^{*} P<0.05,{ }^{* *} P<0.01$, and ${ }^{* * *} P<0.001$. All data included in the same graph were analyzed for type of data distribution with the Shapiro-Wilk normality test. If datasets were normally distributed, then one-way ANOVA was used for multiple comparisons, followed by Bonferroni correction. If the datasets were non-parametric, then the Kruskal-Wallis test was used to analyze variance, followed by pairwise comparisons using the Student's unpaired two-tailed t-test for normally distributed datasets or the Mann-Whitney two-tailed u-test for non-normally distributed data. Then, to correct for Type I error, the Hochberg test was run on $\mathrm{P}$ values compared in the same graph to adjust the $\mathrm{P}$ values for multiple comparisons, which often conservatively increases $\mathrm{P}$ values to avoid incorrectly rejecting the null hypothesis. For graphs with only two datasets to compare, the Shapiro-Wilk test was performed, followed by the t-test or u-test, depending on the distribution of the datasets. For correlation data, the Shapiro-Wilk normality test was run, followed by the Spearman's correlation test. S.E.M. was calculated and shown on each graph, except in Figure 4C where S.D. was used and in Figure 5 where none was shown. The total number of trials is represented by dots on every graph. Specific statistical tests used for each graph in the manuscript are included in the figure legends.

\section{Mean total quiescence analysis}

For analyzing quiescence, the total quiescence in minutes was taken from each data set (analyzed with our Matlab script) and the mean was taken for each data set.

\section{Quiescence disruption analysis}

Videos were scored manually and blinded to experimental treatment. At least two individuals scored each video. After scoring, the measured variables were ranked and averaged across rank to derive a population of scores per video. Specifically, we looked at how many frames passed until the animal completed its first complete sinusoidal bend and how many sinusoidal bends were completed within a set number of frames, both post one second of blue light with constant buzzing treatment. We did not distinguish between forward or backward crawling. 


\section{Calcium imaging analysis}

Fiji software was used with the Multi Measure plugin to analyze the images. In animals expressing the GCaMP3 reporter in the AWCON neuron (JZ1795/pyls701(pstr-2::GCaMP3; pofm$1:: G F P ; p c e h-36:: m$ Cherry)), the ROI was established at the center of the AWC cell body. A background ROI was also taken, just outside of the animals. Then, the mean fluorescence intensity at the background ROI was subtracted from the mean fluorescence intensity at the cell body ROI and that serves as the "F" values. The fluorescence intensity of the GCaMP3 reporter in the first three images is defined as $F_{0}$. Delta $F$ is the $F_{0}$ value subtracted from each $F$ value. For every worm imaged, the mean of the delta $\mathrm{F}_{0} / \mathrm{F}(\%)$ values is taken from the 10 seconds before and after the BTN is turned on or off (i.e. the means at $20-30$ seconds and $30.5-40.5$ seconds are taken). The delta is then taken between the two means and the absolute value is taken of that number for comparisons between the datasets (e.g. buffer vs butanone-trained cohorts) taken on the same day.

\section{Synapse imaging analysis}

NIH ImageJ software (Abràmoff et al., 2004) was used to analyze all micrographs taken for AWC-AIY NLG-1 GRASP phenotypic quantification, as previously described (Park et al., 2011; Varshney et al., 2018). In brief, AWC-AIY NLG-1 GRASP intensity was quantified by measuring fluorescence intensity through circling punctal clusters. In Figure 7E, median intensity values for each treatment were normalized to fluorescence intensity levels for buffer-trained animals that recovered on food for 16 hours. For Figure 7F, median intensity values were normalized to buffer-trained levels taken immediately after training.

PHB-AVA NLG-1 GRASP intensity was also measured through outlining clusters of puncta. Background fluorescence intensity was also taken into account by calculating the value of minimum intensity in the area directly surrounding the puncta. This background intensity value was subtracted from the intensity for each pixel in the punctal cluster, and the adjusted values were added.

NLG-1 GRASP analysis was conducted on all buffer-trained batches that had chemotaxis indices above 0.5 after training. The same criteria were used for butanone-trained batches that recovered off food for two hours. Analysis was also conducted on butanone-trained worms with chemotaxis indices of less than 0.5 . Micrographs were not analyzed if the animals moved during imaging or were damaged in the process of making the slide.

For synapse statistical analysis, the Kruskal-Wallis test was used to analyze variance between treatments, If the $\mathrm{P}$ value was less than 0.05 , then the Mann-Whitney u-test was used to compare the medians of each pair of groups, followed by the Hochberg multiple comparison procedure. 


\section{DATA AND CODE AVAILABILITY}

The code generated during this study is available at www.github.com/letoilelab for the quiescence disruption protocol and https://github.com/cfangyen/LEtoile_WorMotel for the WorMotel quiescence analysis.

\section{ADDITIONAL RESOURCES}

Link to download WorMotel detailed protocol: http://fangyenlab.seas.upenn.edu/links.html

\section{Supplemental Information titles and legends}

\section{Figure S1. Related to Figure 1. Schematic for assessing the chemotaxis assay.}

One-day old adults are washed from NGM plates into two different tubes, where one population will be soaked in buffer and the second will be soaked in butanone diluted in buffer $(1: 10,000)$ for 80 minutes. The animals are then washed with buffer two times, with a third wash in $\mathrm{ddH}_{2} \mathrm{O}$, and are subsequently plated on chemotaxis plates with two odor point sources, where CTL is the diluent and odor is butanone at a 1:1000 dilution. Each odor point source has NaAz as a paralytic. Animals are plated at the origin. After roaming for at least two hours, the chemotaxis index $(\mathrm{Cl})$ can be calculated, which is the number of animals at the CTL arena (indicated by a black box on the left) subtracted from the number at the odor arena (black box on the right), divided by the total number of worms, not counting any worms at the origin. The higher the $\mathrm{Cl}$, the more attracted the worms are to the butanone.

Figure S2. Related to Figure 1. One cycle of butanone training induces memory that is lost only after $\mathbf{3 0}$ minutes of recovery on food.

Chemotaxis indices of animals conditioned for 80 minutes to CTL (light gray bars with gray dots) or BTN (light red bars with gray dots) after 0 hr, 30', 120', or $16 \mathrm{hr}$ of recovery on food. One-way ANOVA was performed $(\mathrm{P}<0.0001)$, followed by the Bonferroni correction for multiple comparisons for CTL vs BTN 0 hr, CTL vs BTN 30', CTL vs BTN 120', CTL vs BTN 16 hr, BTN 0 hr vs BTN 30', BTN 0 hr vs BTN 120', BTN 30' vs BTN 120', and BTN 120' vs BTN 16 hr.

Figure S3. Related to Figure 1. C. elegans can acquire and maintain memory of the AWAsensed odorant diacetyl. Chemotaxis indices of animals spaced-trained to CTL or diacetyl (DIA, red 
bars with teal dots) after $0 \mathrm{hr}$ or $16 \mathrm{hr}$ of recovery on food. One-way ANOVA was performed $(\mathrm{P}<0.0001)$, followed by the Bonferroni correction for multiple comparisons for CTL vs DIA $0 \mathrm{hr}, \mathrm{CTL} 0$ hr vs CTL $16 \mathrm{hr}$, DIA $0 \mathrm{hr}$ vs DIA $16 \mathrm{hr}$, and CTL vs DIA $16 \mathrm{hr}$.

Figure S4. Related to Figure 1. CREB mutants acquire butanone memory, but may not maintain it 16 hours post recovery on food.

Chemotaxis indices of wild-type or crh-1(tz2)/CREB animals spaced-trained to CTL or BTN (where teal bars with gray dots are CTL crh-1/CREB and pink bars with gray dots are BTN crh-1/CREB) after $0 \mathrm{hr}$ and $16 \mathrm{hr}$ of recovery on food. The t-test was performed on CTL vs BTN $0 \mathrm{hr}, \mathrm{crh}-1 / C R E B C T L$ vs crh-1/CREB BTN $0 \mathrm{hr}$, CTL vs BTN $16 \mathrm{hr}$, and BTN vs crh-1/CREB BTN $16 \mathrm{hr}$. The u-test was performed on crh-1/CREB CTL vs crh-1/CREB BTN $16 \mathrm{hr}$, and CTL vs crh-1/CREB CTL $16 \mathrm{hr}$.

Figure S5. Related to Figure 4. Mean total quiescence post training slightly correlates with maintaining the butanone memory 16 hours later.

Correlation analysis was performed between the percentage of animals in a population that sleep during the hour post training and their odor memory 16 hours after training. Sleep was assessed by the high throughput WorMotel analysis as mean total quiescence in minutes per hour, and the memory was assessed by the chemotaxis index of worms trained the same day as the WorMotel analysis after 16 hours of recovery on food. Populations of animals were assayed on independent days. Each dot represents $N=24$ animals for mean total quiescence and $400>N>50$ for chemotaxis index assays. The Spearman's correlation test was performed (for non-normally distributed data), with $r=-0.3914$ and ${ }^{*} P<0.05$.

Figure S6. Related to Figure 7. PHB-AVA synapses are not significantly different among butanone-trained and buffer-trained animals whether or not they are allowed to sleep. (A) Schematic and micrographs of a buffer-trained animal carrying the PHB-AVA NLG-1 GRASP marker with the PHB neurons labeled with the red cytosolic mCherry fluorophore. Synaptic fluorescence is observed in a punctate pattern across the region of PHB-AVA axon overlap. (B) Quantification of PHB-AVA NLG-1 GRASP fluorescence intensity in animals trained with buffer (CTL) or butanone (BTN) and recovered on food for 16 hours, or trained with buffer or butanone and recovered off food for the first two hours, then transferred to plates with food for the following 14 hours. NS, not significant, $p>0.05$, Kruskal-Wallis test. 


\section{Figure S7. Related to Figure 7. Chemotaxis indices for animals carrying NLG-1 GRASP}

\section{markers.}

(A) Chemotaxis indices of AWC-AIY NLG-1 GRASP-carrying animals trained for Figure 7E.

Butanone-trained animals that recovered on food for 16 hours have significantly lower chemotaxis indices than similarly treated buffer-trained animals.

(B) Chemotaxis indices of AWC-AIY NLG-1 GRASP-carrying animals trained for Figure 7F.

Butanone-trained animals at each timepoint have significantly lower chemotaxis indices than similarly treated buffer-trained animals.

(C) Chemotaxis indices of PHB-AVA NLG-1 GRASP-carrying animals trained for Figure S7.

Butanone-trained animals that recovered on food for 16 hours have significantly lower chemotaxis indices than similarly treated buffer-trained animals. ${ }^{* * *} P<0.001$, ${ }^{*} P<0.05$, NS not significant $P>0.05$, t-test, followed by the Hochberg method.

\section{References}

Abdou, K., Shehata, M., Choko, K., Nishizono, H., Matsuo, M., Muramatsu, S., and Inokuchi, K. (2018). Synapse-specific representation of the identity of overlapping memory engrams. Science 360, $1227-1231$.

Abràmoff, M.D., Magelhaes, P.J., and Ram, S.J. (2004). Image Processing with ImageJ. Biophotonics International 11, 36-42.

Acosta-peña, E., Camacho-Abrego, I., Melgarejo-Gutierrez, M., Flores, G., Drucker-Colin, R., and Garcia-Garcia, F. (2015). Sleep deprivation induces differential morphological changes in the hippocampus and prefrontal cortex in young and old rats. Synapse 69, 15-25.

van Alphen, B., Yap, M.H.W., Kirszenblat, L., Kottler, B., and van Swinderen, B. (2013). A Dynamic Deep Sleep Stage in Drosophila. Journal of Neuroscience 33, 6917-6927.

Altun-Gultekin, Z., Andachi, Y., Tsalik, E.L., Pilgrim, D., Kohara, Y., and Hobert, O. (2001). A regulatory cascade of three homeobox genes, ceh-10, ttx-3 and ceh-23, controls cell fate specification of a defined interneuron class in C. elegans. Development 128, 1951-1969.

Appelbaum, L., Wang, G., Yokogawa, T., Skariah, G.M., Smith, S.J., Mourrain, P., and Mignot, E. (2010). Circadian and Homeostatic Regulation of Structural Synaptic Plasticity in Hypocretin Neurons. Neuron 68, 87-98.

Ardiel, E.L., and Rankin, C.H. (2010). An elegant mind: learning and memory in Caenorhabditis elegans. Learning and Memory 17, 191-201.

Arun Asok, Leroy, F., Rayman, J.B., and Kandel, E.R. (2019). Molecular Mechanisms of the Memory Trace. Trends in Neurosciences 42, 14-22.

Bargmann, C.I., and Marder, E. (2013). From the connectome to brain function. Nature Methods 10 , 483-490. 
Bargmann, C.I., Hartweig, E., and Horvitz, H.R. (1993). Odorant-selective genes and neurons mediate olfaction in C. elegans. Cell 74, 515-527.

Beck, C.D.O., Schroeder, B., and Davis, R.L. (2000). Learning Performance of Normal and Mutant Drosophila after Repeated Conditioning Trials with Discrete Stimuli. J. Neurosci. 20, 2944-2953.

Beyaert, L., Greggers, U., and Menzel, R. (2012). Honeybees consolidate navigation memory during sleep. Journal of Experimental Biology 215, 3981-3988.

Bono, M. de, and Villu Maricq, A. (2005). Neuronal Substrates of Complex Behaviors in C. elegans. Annu. Rev. Neurosci. 28, 451-501.

de Bono, M., and Maricq, A.V. (2005). Neuronal Substrates of Complex Behaviors in C. elegans. Annual Review of Neuroscience 28, 451-501.

Brawn, T.P., Nusbaum, H.C., and Margoliash, D. (2018). Sleep-dependent reconsolidation after memory destabilization in starlings. Nature Communications 9, 3093.

Bushey, D., Tononi, G., and Cirelli, C. (2011). Sleep and Synaptic Homeostasis: Structural Evidence in Drosophila. Science 332, 1576-1581.

Butler, H.E. (1921). Quintilian's Institutio Oratoria, Loeb Classical Library (Harvard University Press).

Chalasani, S.H., Chronis, N., Tsunozaki, M., Gray, J.M., Ramot, D., Goodman, M.B., and Bargmann, C.I. (2007). Dissecting a circuit for olfactory behaviour in Caenorhabditis elegans. Nature 450, 63-70.

Chauvette, S., Seigneur, J., and Timofeev, I. (2012). Sleep oscillations in the thalamocortical system induce long-term neuronal plasticity. Neuron 75, 1105-1113.

Cho, C.E., Brueggemann, C., L'Etoile, N.D., and Bargmann, C.I. (2016). Parallel encoding of sensory history and behavioral preference during Caenorhabditis elegans olfactory learning. ELife 5, e14000.

Choi, S., Chatzigeorgiou, M., Taylor, K.P., Schafer, W.R., and Kaplan, J.M. (2013). Analysis of NPR-1 reveals a circuit mechanism for behavioral quiescence in C. elegans. Neuron 78, 869-880.

Chronis, N., Zimmer, M., and Bargmann, C.I. (2007). Microfluidics for in vivo imaging of neuronal and behavioral activity in Caenorhabditis elegans. Nature Methods 4, 727-731.

Churgin, M.A., Jung, S.-K., Yu, C.-C., Chen, X., Raizen, D.M., and Fang-Yen, C. (2017). Longitudinal imaging of Caenorhabditis elegans in a microfabricated device reveals variation in behavioral decline during aging. ELife 6, e26652.

Colbert, H., and Bargmann, C.I. (1995). Odorant-specific adaptation pathways generate olfactory plasticity in C. elegans. Neuron 14, 803-812.

Cook, S.J., Jarrell, T.A., Brittin, C.A., Wang, Y., Bloniarz, A.E., Yakovlev, M.A., Nguyen, K.C.Q., Tang, L.T.-H., Bayer, E.A., Duerr, J.S., et al. (2019). Whole-animal connectomes of both Caenorhabditis elegans sexes. Nature 571, 63-71.

Cowansage, K.K., Shuman, T., Dillingham, B.C., Chang, A., Golshani, P., and Mayford, M. (2014). Direct Reactivation of a CoherentNeocortical Memory of Context. Neuron 84, 432-441. 
Diering, G.H., Nirujogi, R.S., Roth, R.R., Worley, P.F., Pandey, A., and Huganir, R.L. (2017). Homer1a drives homeostatic scaling-down of excitatory synapses during sleep. Science 355, 511515.

Donlea, J.M., Ramanan, N., and Shaw, P.J. (2009). Use-dependent plasticity in clock neurons regulates sleep need in Drosophila. Science 324, 105-108.

Dudai, Y., Karni, A., and Born, J. (2015). The Consolidation and Transformation of Memory. Neuron 88, 20-32.

Durkin, J., and Aton, S.J. (2016). Sleep-Dependent Potentiation in the Visual System Is at Odds with the Synaptic Homeostasis Hypothesis. Sleep 39, 155-159.

Edelstein, A.D., Tsuchida, M.A., Amodaj, N., Pinkard, H., and Vale, R.D. (2014). Advanced methods of microscope control using $\mu$ Manager software. Journal of Biological Methods 1, e10.

Estes, W.K. (1955). Statistical theory of distributional phenomena in learning. Psychological Review 62, 369-377.

Feinberg, E., VanHoven, M.K., Bendesky, A., Wang, G., Fetter, R.D., Shen, K., and Bargmann, C.I. (2008). GFP Reconstitution Across Synaptic Partners (GRASP) Defines Cell Contacts and Synapses in Living Nervous Systems. Neuron 57, 353-363.

Fox, K., and Stryker, M. (2017). Integrating Hebbian and homeostatic plasticity: introduction. Philosophical Transactions of the Royal Society B 372.

Gallagher, T., Kim, J., Oldenbroek, M., Kerr, R., and You, Y. (2013). ASI regulates satiety quiescence in C. elegans. The Journal of Neuroscience 33, 9716-9724.

Gilestro, G.F., Tononi, G., and Cirelli, C. (2009). Widespread changes in synaptic markers as a function of sleep and wakefulness in Drosophila. Science 324, 109-112.

Gordus, A., Pokala, N., Levy, S., Flavell, S.W., and Bargmann, C.I. (2015). Feedback from network states generates variability in a probabilistic olfactory circuit. Cell 161, 215-227.

Grosmark, A.D., Mizuseki, K., Pastalkova, E., Diba, K., and Buzsáki, G. (2012). REM Sleep Reorganizes Hippocampal Excitability. Neuron 75, 1001-1007.

Hadziselimovic, N., Vukojevic, V., Peter, F., Milnik, A., Fastenrath, M., Gabor Fenyes, B., Hieber, P., Fastenrath, P., Vogler, C., de Quervain, D.J.-F., et al. (2014). Forgetting is regulated via Musashimediated translational control of the Arp2/3 complex. Cell 156, 1153-1166.

Hengen, K.B., Torrado Pacheco, A., McGregor, J.N., Van Hooser, S.D., and Turrigiano, G.G. (2016). Neuronal Firing Rate Homeostasis Is Inhibited by Sleep and Promoted by Wake. Cell 165, 180-191.

Hill, A.J., Mansfield, R., Lopez, J.M.N.G., Raizen, D.M., and Van Buskirk, C. (2014). Cellular stress induces a protective sleep-like state in C. elegans. Current Biology 24, 2399-2405.

Hilliard, M.A., Bargmann, C.I., and Bazzicalupo, P. (2002). C. elegans Responds to Chemical Repellents by Integrating Sensory Inputs from the Head and the Tail. Current Biology 12, 730-734.

Hussaini, S.A., Bogusch, L., Landgraf, T., and Menzel, R. (2009). Sleep deprivation affects extinction but not acquisition memory in honeybees. Learning and Memory 16, 698-705. 
Ishihara, T., lino, Y., Mohri, A., Mori, I., Gengyo-Ando, K., Mitani, S., and Katsura, I. (2002). HEN-1, a Secretory Protein with an LDL Receptor Motif, Regulates Sensory Integration and Learning in Caenorhabditis elegans. Cell 109, 639-649.

Jackson, C., McCabe, B.J., Nicol, A.U., Grout, A.S., Brown, M.W., and Horn, G. (2008). Dynamics of a Memory Trace: Effects of Sleep on Consolidation. Current Biology 18, 393-400.

Jan Born, and Feld, G.B. (2012). Sleep to Upscale, Sleep to Downscale: Balancing Homeostasis and Plasticity. Neuron 75, 933-935.

Kandel, E.R. (2012). The molecular biology of memory: cAMP, PKA, CRE, CREB-1, CREB-2, and CPEB. Mol Brain 5, 14.

Kaufmann, A.L., Ashraf, J.M., Corces-Zimmerman, R., Landis, J.N., and Murphy, C.T. (2010). Insulin Signaling and Dietary Restriction Differentially Influence the Decline of Learning and Memory with Age. PLOS Biology 8, e1000372.

Kimura, Y., Corcoran, E.E., Eto, K., Gengyo-Ando, K., Muramatsu, M. -a., Kobayashi, R., Freedman, J.H., Mitani, S., Hagiwara, M., Means, A.R., et al. (2002). A CaMK cascade activates CRE-mediated transcription in neurons of Caenorhabditis elegans. EMBO Reports 3, 962-966.

Kochanek, K.D., Murphy, S.L., Xu, J., and Arias, E. (2017). Mortality in the United States, 2016. NCHS Data Brief (CDC) 8.

Kreutzmann, J.C., Havekes, R., Abel, T., and Meerlo, P. (2015). Sleep deprivation and hippocampal vulnerability: changes in neuronal plasticity, neurogenesis and cognitive function. Neuroscience 309, 173-190.

Lakhina, V., Arey, R.N., Kaletsky, R., Kauffman, A., Stein, G., Keyes, W., Xu, D., and Murphy, C.T. (2015). Genome-wide Functional Analysis of CREB/Long-Term Memory-Dependent Transcription Reveals Distinct Basal and Memory Gene Expression Programs. Neuron 85, 330-345.

Lee, D.A., Andreev, A., Truong, T.V., Chen, A., Hill, A.J., Oikonomou, G., Pham, U., Hong, Y.K., Tran, S., Glass, L., et al. (2017). Genetic and neuronal regulation of sleep by neuropeptide VF. ELife 6, e25727.

Lenz, O., Xiong, J., Nelson, M.D., Raizen, D.M., and Williams, J.A. (2015). FMRFamide signaling promotes stress-induced sleep in Drosophila. Brain, Behavior, and Immunity 47, 141-148.

L'Etoile, N.D., and Bargmann, C.I. (2000). Olfaction and Odor Discrimination Are Mediated by the C. elegans Guanylyl Cyclase ODR-1. Neuron 25, 575-586.

Li, Z., Liu, J., Zheng, M., and Xu, X.Z.S. (2014). Encoding of Both Analog- and Digital-like Behavioral Outputs by One C. elegans Interneuron. Cell 159, 751-765.

Lin, M.-S., Xiong, W.-C., Li, S.-J., Gong, Z., Cao, X., Kuang, X.-J., Zhang, Y., Gao, T.-M., Mechawar, N., Liu, C., et al. (2017). a2-glycine receptors modulate adult hippocampal neurogenesis and spatial memory: a2-GlyRs Modulate Adult Hippocampal Neurogenesis. Devel Neurobio 77, 1430-1441.

Liu, Z.-W., Faraguna, U., Cirelli, C., Tononi, G., and Gao, X.-B. (2010). Direct evidence for wakerelated increases and sleep-related decreases in synaptic strength in rodent cortex. JNeurosci 30 , 8671-8675. 
Lonze, B.E., and Ginty, D.D. (2002). Function and Regulation of CREB Family Transcription Factors in the Nervous System. Neuron 35, 605-623.

Loria, P.M. (2004). A Conserved Postsynaptic Transmembrane Protein Affecting Neuromuscular Signaling in Caenorhabditis elegans. Journal of Neuroscience 24, 2191-2201.

Maret, S., Faraguna, U., Nelson, A.B., Cirelli, C., and Tononi, G. (2011). Sleep and wake modulate spine turnover in the adolescent mouse cortex. Nature Neuroscience 14, 1418-1420.

Mauelshagen, J., Sherff, C.M., and Carew, T.J. (1998). Differential Induction of Long-Term Synaptic Facilitation by Spaced and Massed Applications of Serotonin at Sensory Neuron Synapses of Aplysia californica. Learning and Memory 5, 246-256.

McGaugh, J.L. (1966). Time-Dependent Processes in Memory Storage. Science 153, 1351-1358.

Meeusen, T., Mertens, I., Clynen, E., Baggerman, G., Nichols, R., Nachman, R.J., Huybrechts, R., Loof, A.D., and Schoofs, L. (2002). Identification in Drosophila melanogaster of the invertebrate G protein-coupled FMRFamide receptor. 6.

Menzel, R., Leboulle, G., and Eisenhardt, D. (2006). Small brains, bright minds. Cell 124, $237-239$.

Mori, I., and Oshima, Y. (1995). Neural regulation of thermotaxis in Caenorhabditis elegans. Nature 376, 344-376.

Nagel, G., Brauner, M., Liewald, J.F., Adeishvili, N., Bamberg, E., and Gottschalk, A. (2005). Light activation of channelrhodopsin-2 in excitable cells of Caenorhabditis elegans triggers rapid behavioral responses. Current Biology 15, 2279-2284.

Nagy, S., Tramm, N., Sanders, J., Iwanir, S., Shirley, I.A., Levine, E., and Biron, D. (2014). Homeostasis in $\mathrm{C}$. elegans sleep is characterized by two behaviorally and genetically distinct mechanisms. ELife 3.

Nath, R.D., Chow, E.S., Wang, H., Schwarz, E.M., and Sternberg, P.W. (2016). C. elegans StressInduced Sleep Emerges from the Collective Action of Multiple Neuropeptides. Current Biology 26, 2446-2455.

Nelson, M.D., Trojanowski, N.F., George-Raizen, J.B., Smith, C.J., Yu, C.-C., Fang-Yen, C., and Raizen, D.M. (2013). The neuropeptide NLP-22 regulates a sleep-likestate in Caenorhabditis elegans. Nature Communications 4, Article 2846.

Nelson, M.D., Lee, K.H., Churgin, M.A., Hill, A.J., Van Buskirk, C., Fang-Yen, C., and Raizen, D.M. (2014). FMRFamide-like FLP-13 neuropeptides promote quiescence following heat stress in Caenorhabditis elegans. Current Biology 24, 2406-2410.

Nichols, A.L.A., Eichler, T., Latham, R., and Zimmer, M. (2017). A global brain state underlies C. elegans sleep behavior. Science 356, eaam6851.

Park, J., Knezevich, P.L., Wung, W., O’Hanlon, S.N., Goyal, A., Benedetti, K.L., Barsi-Rhyne, B.J., Raman, M., Mock, N., Bremer, M., et al. (2011). A conserved juxtacrine signal regulates synaptic partner recognition in C. elegans. Neural Development 6, 28.

Pierce-Shimomura, J.T., Morse, T.M., and Lockery, S.R. (1999). The Fundamental Role of Pirouettes in Caenorhabditis elegans Chemotaxis. J. Neurosci. 19, 9557-9569. 
Raizen, D.M., Zimmerman, J.E., Maycock, M.H., Ta, U.D., You, Y., Sundaram, M.V., and Pack, A.I. (2008). Lethargus is a Caenorhabditis elegans sleep-like state. Nature 451, 569-572.

Raizen, D.M., Song, B., Trojanowski, N.F., and You, Y. (2012). Methods for measuring pharyngeal behaviors. WormBook.

Rasch, B., and Born, J. (2013). About Sleep’s Role in Memory. Physiological Reviews 93, 681-766.

Raven, F., Van der Zee, E.A., Meerlo, P., and Havekes, R. (2018). The role of sleep in regulating structural plasticity and synaptic strength: Implications for memory and cognitive function. Sleep Medicine Reviews 39, 3-11.

Rubinski, A., and Ziv, N.E. (2015). Remodeling and Tenacity of Inhibitory Synapses: Relationships with Network Activity and Neighboring Excitatory Synapses. PLOS Computational Biology 11, e1004632.

Ryan, T.J., Roy, D.S., Pignatelli, M., Arons, A., and Tonegawa, S. (2015). Memory. Engram cells retain memory under retrograde amnesia. Science 348, 1007-1013.

Schwarz, J., Spies, J.-P., and Bringmann, H. (2012). Reduced muscle contraction and a relaxed posture during sleep-like Lethargus. Worm 1, 12-14.

Sengupta, P., Chou, J.H., and Bargmann, C.I. (1996). odr-10 encodes a seven transmembrane domain olfactory receptor required for responses to the odorant diacetyl. Cell 84, 899-909.

Silva, A.J., Kogan, J.H., Frankland, P.W., and Kida, S. (1998). CREB and Memory. Annual Review of Neuroscience 21, 127-148.

Singh, K., Ju, J.Y., Walsh, M.B., and Dilorio, M.A. (2014). Deep conservation of genes required for both Drosphila melanogaster and Caenorhabditis elegans sleep includes a role for dopaminergic signaling. Sleep 37, 1439-1451.

Skora, S., Mende, F., and Zimmer, M. (2018). Energy Scarcity Promotes a Brain-wide Sleep State Modulated by Insulin Signaling in C. elegans. Cell Reports 22, 953-966.

Stiernagle, T. (2006). Maintenance of C. elegans. WormBook.

Tian, L., Hires, S.A., Mao, T., Chiappe, M.E., Chalasani, S.H., Petreanu, L., Akerboom, J., McKinney, S.A., Schreiter, E.R., Bargmann, C.I., et al. (2009). Imaging neural activity in worms, flies and mice with improved GCaMP calcium indicators. Nature Methods 6, 875-881.

Tononi, G., and Cirelli, C. (2014). Sleep and the Price of Plasticity: From Synaptic and Cellular Homeostasis to Memory Consolidation and Integration. Neuron 81, 12-34.

Torayama, I., Ishihara, T., and Katsura, I. (2007). Caenorhabditis elegans Integrates the Signals of Butanone and Food to Enhance Chemotaxis to Butanone. Journal of Neuroscience 27, 741-750.

Tramm, N., Oppenheimer, N., Nagy, S., Efrati, E., and Biron, D. (2014). Why Do Sleeping Nematodes Adopt a Hockey-Stick-Like Posture? PLOS ONE 9, e101162.

Tran, A., Tang, A., O’Loughlin, C.T., Balistreri, A., Chang, E., Coto Villa, D., Li, J., Varshney, A., Jimenez, V., Pyle, J., et al. (2017). C. elegans avoids toxin-producing Streptomyces using a seven transmembrane domain chemosensory receptor. ELife 6, e23770. 
bioRxiv preprint doi: https://doi.org/10.1101/2020.11.24.395228; this version posted April 8, 2021. The copyright holder for this preprint (which was not certified by peer review) is the author/funder, who has granted bioRxiv a license to display the preprint in perpetuity. It is made available under aCC-BY-ND 4.0 International license.

Trojanowski, N.F., and Raizen, D.M. (2016). Call it Worm Sleep. Trends in Neurosciences 39, 54-62.

Trojanowski, N.F., Nelson, M.D., Flavell, S.W., Fang-Yen, C., and Raizen, D.M. (2015). Distinct Mechanisms Underlie Quiescence during Two Caenorhabditis elegans Sleep-Like States. The Journal of Neuroscience 35, 14571-14584.

Tsunozaki, M., Chalasani, S.H., and Bargmann, C.I. (2008). A Behavioral Switch: cGMP and PKC Signaling in Olfactory Neurons Reverses Odor Preference in C. elegans. Neuron 59, 959-971.

Turek, M., Besseling, J., Spies, J.-P., Konig, S., and Bringmann, H. (2016). Sleep-active neuron specification and sleep induction require FLP-11 neuropeptides to systemically induce sleep. ELife 5, e12499.

Turrigiano, G. (2017). The Dialectic of Hebb and Homeostasis. Philosophical Transactions of the Royal Society B 372.

Turrigiano, G., Leslie, K.R., Desai, N.S., Rutherford, L.C., and Nelson, S.B. (1998). Activitydependent scaling of quantal amplitude in neocortical neurons. Nature 391, 892-896.

Van Buskirk, C., and Sternberg, P.W. (2007). Epidermal growth factor signaling induces behavioral quiescence in Caenorhabditis elegans. Nature Neuroscience 10, 1300-1307.

Varshney, A., Benedetti, K., Watters, K., Shankar, R., Tatarakis, D., Coto Villa, D., Magallanes, K., Agenor, V., Wung, W., Farah, F., et al. (2018). The receptor protein tyrosine phosphatase CLR-1 is required for synaptic partner recognition. PLOS Genetics 14, e1007312.

de Vivo, L., Bellesi, M., Marshall, W., Bushong, E.A., Ellisman, M.H., Tononi, G., and Cirelli, C. (2017). Ultrastructural evidence for synaptic scaling across the wake/sleep cycle. Science 355, 507510.

Vohra, M., Lemieux, G.A., Lin, L., and Ashrafi, K. (2017). The beneficial effects of dietary restriction on learning are distinct from its effects on longevity and mediated by depletion of a neuroinhibitory metabolite. PLoS Biol 15, e2002032.

Vorster, A.P., and Born, J. (2015). Sleep and memory in mammals, birds and invertebrates. Neuroscience and Biobehavioral Reviews 50, 103-119.

Vyazovskiy, V.V., and Faraguna, U. (2014). Sleep and Synaptic Homeostasis. In Sleep, Neuronal Plasticity and Brain Function, (Springer-Verlag Berlin Heidelberg), pp. 91-121.

Vyazovskiy, V.V., Cirelli, C., Pfister-Genskow, M., Faraguna, U., and Tononi, G. (2008). Molecular and electrophysiological evidence for net synaptic potentiation in wake and depression in sleep. Nature Neuroscience 11, 200-208.

Walker, M.P. (2009). The Role of Sleep in Cognition and Emotion. The Year in Cognitive Neuroscience 2009: Ann. N.Y. Acad. Sc 1156, 168-197.

Wang, D.Y.-C., Kumar, S., and Hedges, S.B. (1999). Divergence time estimates for the early history of animal phyla and the origin of plants, animals and fungi. Proceedings of the Royal Society of London. Series B: Biological Sciences 266, 163-171.

Weber, F. (2017). Modeling the mammalian sleep cycle. Current Opinion in Neurobiology 46, 68-75. 
Wes, P.D., and Bargmann, C.I. (2001). C. elegans odour discrimination requires asymmetric diversity in olfactory neurons. Nature 410, 698-701.

White, J.G., Southgate, E., Thomson, J.N., and Brenner, S. (1986). The structure of the nervous system of the nematode C. elegans. Philosophical Transactions of the Royal Society B 314, 1-340.

Wolstenholme, A.J. (2012). Glutamate-gated Chloride Channels. The Journal of Biological Chemistry 287, 40232-40238.

Worthy, S.E., Rojas, G.L., Taylor, C.J., and Glater, E.E. (2018a). Identification of Odor Blend Used by Caenorhabditis elegans for Pathogen Recognition. Chemical Senses 43, 169-180.

Worthy, S.E., Haynes, L., Chambers, M., Bethune, D., Kan, E., Chung, K., Ota, R., Taylor, C.J., and Glater, E.E. (2018b). Identification of attractive odorants released by preferred bacterial food found in the natural habitats of C. elegans. PLoS One 13, e0201158.

Xu, T.-L., and Gong, N. (2010). Glycine and glycine receptor signaling in hippocampal neurons: Diversity, function and regulation. Progress in Neurobiology 91, 349-361.

Yamaguchi, M. (2017). The role of sleep in the plasticity of the olfactory system. Neuroscience Research 118, 21-29.

Yap, M.H.W., Grabowska, M.J., Rohrscheib, C., Jeans, R., Troup, M., Paulk, A.C., van Alphen, B., Shaw, P.J., and van Swinderen, B. (2017). Oscillatory brain activity in spontaneous and induced sleep stages in flies. Nat Commun 8, 1815.

Yonelinas, A.P., Ranganath, C., Ekstrom, A.D., and Wiltgen, B.J. (2019). A contextual binding theory of episodic memory: systems consolidation reconsidered. Nature Reviews Neuroscience 20, 364375.

You, Y., Kim, J., Raizen, D.M., and Avery, L. (2008). Insulin, cGMP, and TGF- $\beta$ Signals Regulate Food Intake and Quiescence in C. elegans: A Model for Satiety. Cell Metabolism 7, 249-257.

Zhang, Y., Lu, H., and Bargmann, C.I. (2005). Pathogenic bacteria induce aversive olfactory learning in Caenorhabditis elegans. Nature 438, 179-184.

Zimmerman, J.E., Naidoo, N., Raizen, D.M., and Pack, A.I. (2008). Conservation of sleep: insights from non-mammalian model systems. Trends in Neurosciences 31, 371-376. 


\section{KEY RESOURCES TABLE}

\begin{tabular}{|c|c|c|}
\hline REAGENT or RESOURCE & SOURCE & IDENTIFIER \\
\hline \multicolumn{3}{|l|}{ Bacterial and Virus Strains } \\
\hline OP50 E. coli & Caenorhabditis Genetics Center & OP50 \\
\hline \multicolumn{3}{|l|}{$\begin{array}{l}\text { Chemicals, Peptides, and } \\
\text { Recombinant Proteins }\end{array}$} \\
\hline 2-butanone & Sigma-Aldrich & 360473 \\
\hline Sodium azide $99 \%$ & Fisher Scientific, Sigma-Aldrich & ICN10289180, S2002 \\
\hline Benzaldehyde & Sigma-Aldrich & B1334 \\
\hline Diacetyl/2,3-butanedione & Sigma-Aldrich & B85307 \\
\hline Levamisole & Acros Organics & AC187870100 \\
\hline BDM & Fluka Analytical & $31550 / 2003485$ \\
\hline Tween 20 detergent & Millipore & 655204 \\
\hline $\mathrm{NaCl}$ & Fisher Chemical & S671-10 \\
\hline Potassium phosphate dibasic & Fisher Scientific & S375-500 \\
\hline Potassium phosphate monobasic & Sigma-Aldrich & P285 \\
\hline Bacto agar & Difco & $90000-762$ \\
\hline Calcium chloride & Sigma-Aldrich & C8106 \\
\hline Magnesium sulfate & Sigma-Aldrich & M7506 \\
\hline Low melting point agarose & Apex Chemicals and Reagents/Genesee & $20-104$ \\
\hline Bacto peptone & Difco & DF0118-07-2 \\
\hline Cholesterol & Sigma-Aldrich & C3045 \\
\hline $\begin{array}{l}\text { PDMS/ Dow Corning Sylgard } 184 \\
\text { Silicone Encapsulant }\end{array}$ & Ellsworth Adhesives & 4019862 \\
\hline Soil Moist granules & JRM Chemical Inc. & $\mathrm{N} / \mathrm{A}$ \\
\hline 95\% Ethanol & Fisher Scientific & A405P-4 \\
\hline Agarose & Fisher Scientific & BP1356-500 \\
\hline \multicolumn{3}{|l|}{$\begin{array}{l}\text { Experimental Models: } \\
\text { Organisms/Strains }\end{array}$} \\
\hline wild-type Bristol N2 var. & Caenorhabditis Genetics Center (CGC) & N2 \\
\hline $\begin{array}{l}\text { unc-119; goels240 [phsp-16.2::flp- } \\
\text { 11::SL2::mKate2::unc-54 3'UTR + } \\
\text { unc-119(+)] }\end{array}$ & CGC & $\begin{array}{l}{ }_{p} H S:: f f p-11 \text {; strain name: } \\
\text { HBR1021 }\end{array}$ \\
\hline $\begin{array}{l}\text { pels578 (pnpr-9::casp1; punc- } \\
\text { 122::mCherry; pnpr-9::venus) }\end{array}$ & lino lab & AIB kill; strain name: JN578 \\
\hline \multicolumn{3}{|l|}{$t t x-3(k s 5) X$} \\
\hline $\begin{array}{l}\text { ttx-3(ks5) X; pels578 (pnpr- } \\
\text { 9::casp1; punc-122::mCherry; pnpr- } \\
\text { 9::venus) }\end{array}$ & This study & $\begin{array}{l}\text { AIB, AIY double kill; strain } \\
\text { name: JZ2008 }\end{array}$ \\
\hline crh-1(tz2) III & CGC & $\begin{array}{l}\text { crh-1/CREB; strain name: } \\
\text { YT17 }\end{array}$ \\
\hline $\begin{array}{l}\text { wyls155 (pgpa-6::nlg-1::GFP1-10; } \\
\text { pflp-18::nlg-1::GFP11; pnlp- } \\
1:: m \text { Cherry; plp-18::mCherry; podr- } \\
1:: D \text { SRedII) X }\end{array}$ & This study & $\begin{array}{l}\text { PHB-AVA NLG-1 GRASP; } \\
\text { strain name: MKV1058 }\end{array}$ \\
\hline $\begin{array}{l}\text { iyls35 (pttx-3::nlg-1::GFP1-10; } \\
\text { podr-1::nlg-1::GFP11; podr- } \\
\text { 1::DsRedll; punc-122::RFP) III }\end{array}$ & This study & $\begin{array}{l}\text { AWC-AIY NLG-1 GRASP; } \\
\text { strain name: MKV1022 }\end{array}$ \\
\hline
\end{tabular}




\begin{tabular}{|c|c|c|}
\hline $\begin{array}{l}\text { pyls701 (pstr-2::GCaMP3; }{ }_{p} \text { ofm- } \\
1:: G F P ;{ }_{p} \text { ceh-36::mCherry) }\end{array}$ & This study & $\begin{array}{l}{ }_{p}{ } A W C O N:: G C a M P 3 ; \text { strain } \\
\text { name: JZ1795 }\end{array}$ \\
\hline \multicolumn{3}{|l|}{ Oligonucleotides } \\
\hline $\begin{array}{l}\text { MVP578: } \\
\text { TTGCATGCCTGCAGGTCG }\end{array}$ & This study & $\begin{array}{l}\text { Forward primer used to } \\
\text { generate }{ }_{p} \text { odr-1::nlg- } \\
1:: G F P 11\end{array}$ \\
\hline $\begin{array}{l}\text { MVP581: } \\
\text { GACTGGCGCGCCTACCTTTGG } \\
\text { GTCCTTTGGC }\end{array}$ & This study & $\begin{array}{l}\text { Reverse primer used to } \\
\text { generate podr-1::nlg- } \\
1:: G F P 11\end{array}$ \\
\hline \multicolumn{3}{|l|}{ Recombinant DNA } \\
\hline$p t t x-3:: n \lg -1:: G F P 1-10$ & Feinberg et al., 2008 & Used to generate iyls35 \\
\hline podr-1::nlg-1::GFP11 & This study & Used to generate iyls35 \\
\hline podr-1::DsRedII & (L'Etoile and Bargmann, 2000) & Used to generate iyls35 \\
\hline punc-122::RFP & (Loria et al., 2004) & $\begin{array}{l}\text { Used to generate iyls } 35 \text { and } \\
\text { wyls } 155\end{array}$ \\
\hline pgpa-6::nlg-1::GFP1-10 & (Park et al., 2011) & Used to generate wyls155 \\
\hline pflp-18::nlg-1::GFP11 & (Park et al., 2011) & Used to generate wyls155 \\
\hline${ }_{p} n l p-1:: m$ Cherry & (Park et al., 2011) & Used to generate wyls155 \\
\hline pflp-18::mCherry & (Park et al., 2011) & Used to generate wyls155 \\
\hline \multicolumn{3}{|l|}{ Software and Algorithms } \\
\hline Prism 8 & Graphpad & $\begin{array}{l}\text { https://www.graphpad.com/sc } \\
\text { ientificsoftware/ } \\
\text { prism/ }\end{array}$ \\
\hline ImageJ & $\mathrm{NIH}$ & $\begin{array}{l}\text { https://imagej.nih.gov/ij/downl } \\
\text { oad.html }\end{array}$ \\
\hline Fiji & Fiji contributors & https://imagej.net/Fiji \\
\hline RStudio & RStudio & $\begin{array}{l}\text { https://www.rstudio.com/prod } \\
\text { ucts/rstudio/\#Desktop }\end{array}$ \\
\hline Axiovision & Zeiss & $\begin{array}{l}\text { https://www.zeiss.com/micros } \\
\text { copy/us/products/microscope- } \\
\text { software/axiovision.html }\end{array}$ \\
\hline Multi-Worm Tracker & Rex Kerr & $\begin{array}{l}\text { https://sourceforge.net/project } \\
\text { s/mwt/ }\end{array}$ \\
\hline Matlab & MathWorks & $\begin{array}{l}\text { https://www.mathworks.com/p } \\
\text { roducts/matlab.html }\end{array}$ \\
\hline umanager & Ron Vale lab & $\begin{array}{l}\text { https://micro- } \\
\text { manager.org/wiki/Download\% } \\
\text { 20Micro- } \\
\text { Manager_Latest\%20Release }\end{array}$ \\
\hline Irfanview & Irfan Škiljan & $\begin{array}{l}\text { https://download.cnet.com//rf } \\
\text { anView/ }\end{array}$ \\
\hline ARDUINO 1.8 .9 & Arduino & $\begin{array}{l}\text { https://www.arduino.cc/en/Ma } \\
\text { in/Software }\end{array}$ \\
\hline Arduino_blink_buzz & This study & www.GitHub.com/letoilelab \\
\hline KS_analysis_CFY_Jan2019.m & This study & $\begin{array}{l}\text { https://github.com/cfangyen/L } \\
\text { Etoile_WorMotel }\end{array}$ \\
\hline MC_QuiescenceActivity_v1202.fig & This study & $\begin{array}{l}\text { https://github.com/cfangyen/L } \\
\text { Etoile_WorMotel }\end{array}$ \\
\hline
\end{tabular}


A bioRxiv preptint doi: https:///6rgy/10.1101/20207 24.395228; this version posted April

was netcertified by peer(eview) is the authorifunder, who has granted bioRxiv a license to aıspiay tne preprint in perpetuity. It is maae

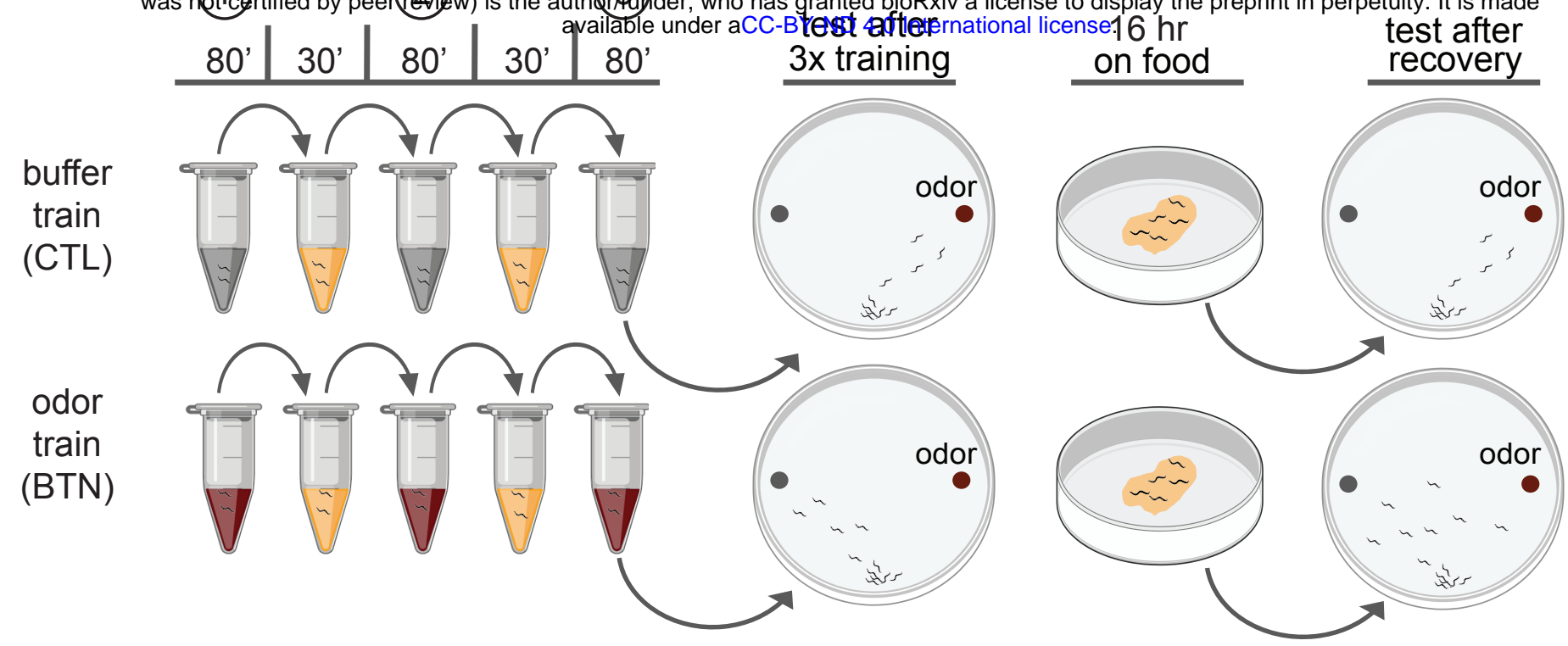

B

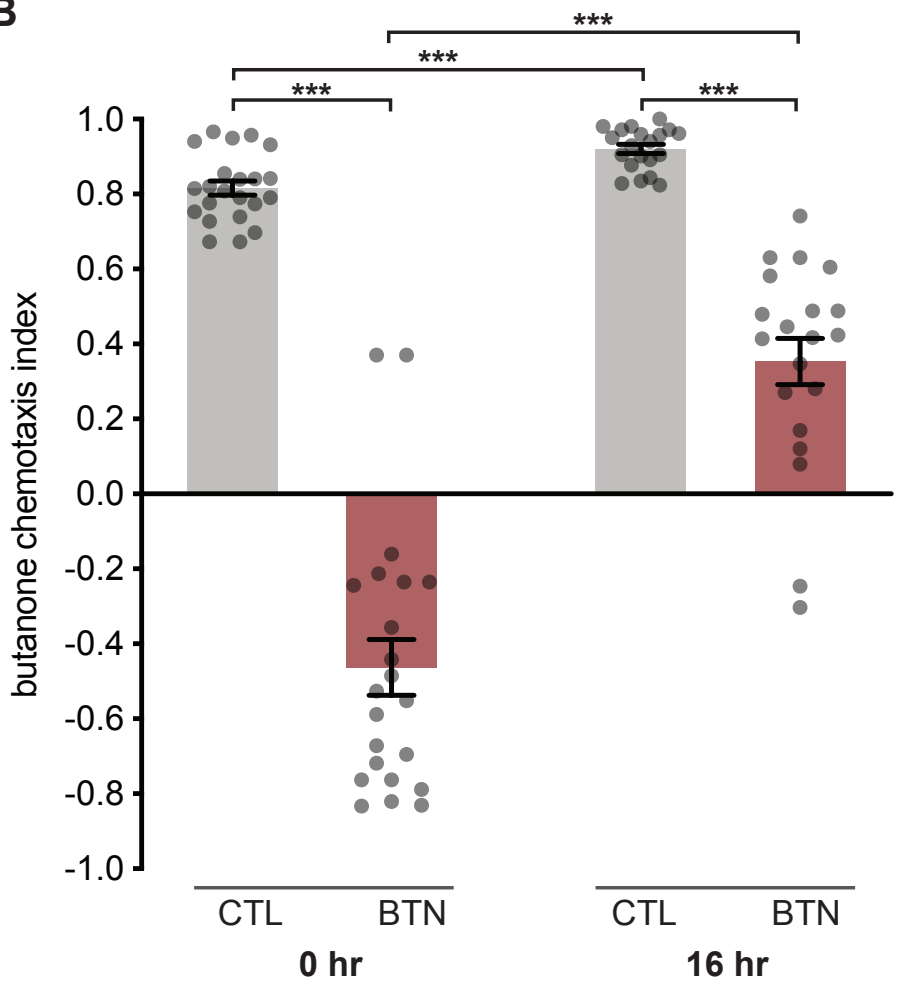

C

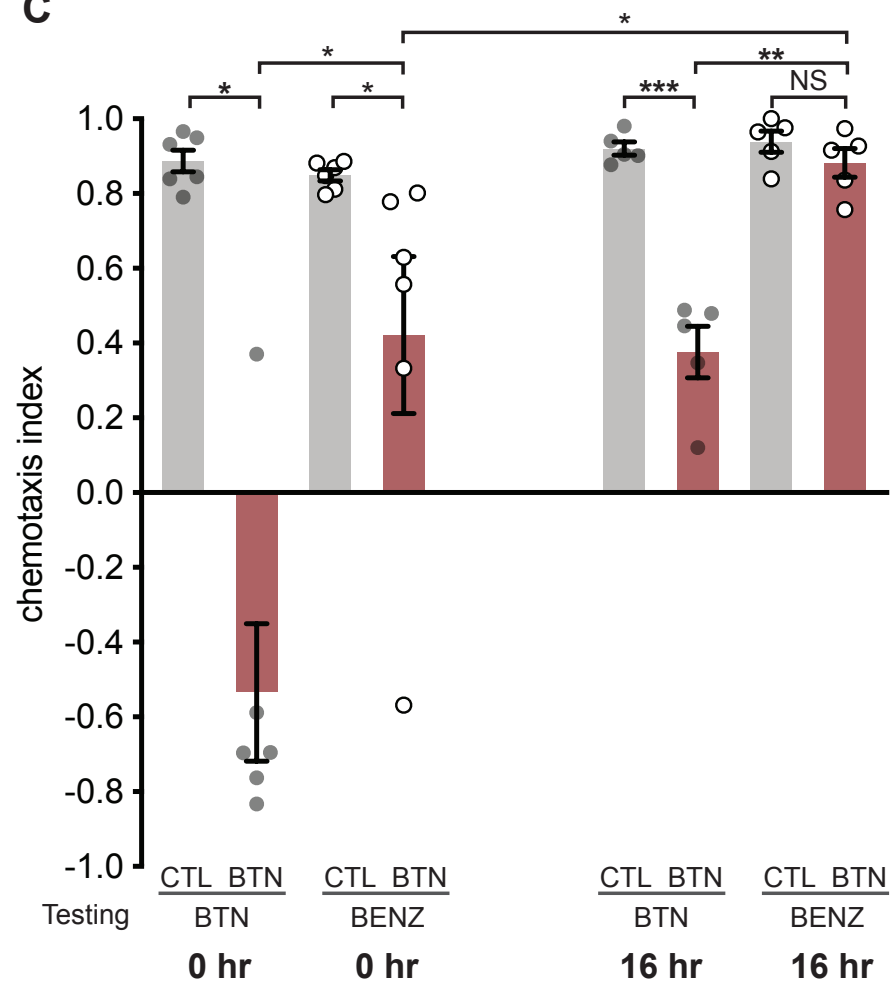


A bioRxiv preprint doi: https://doi.org/10.1101/2020.11.24.395228; this version posted April

A was not certified by peer review) is the author/funder, who has granted bioRxiv a license to aıspıay tne preprınt in perpetuıty. It is maae available under atec-BY-ND 4 O lnternational license.

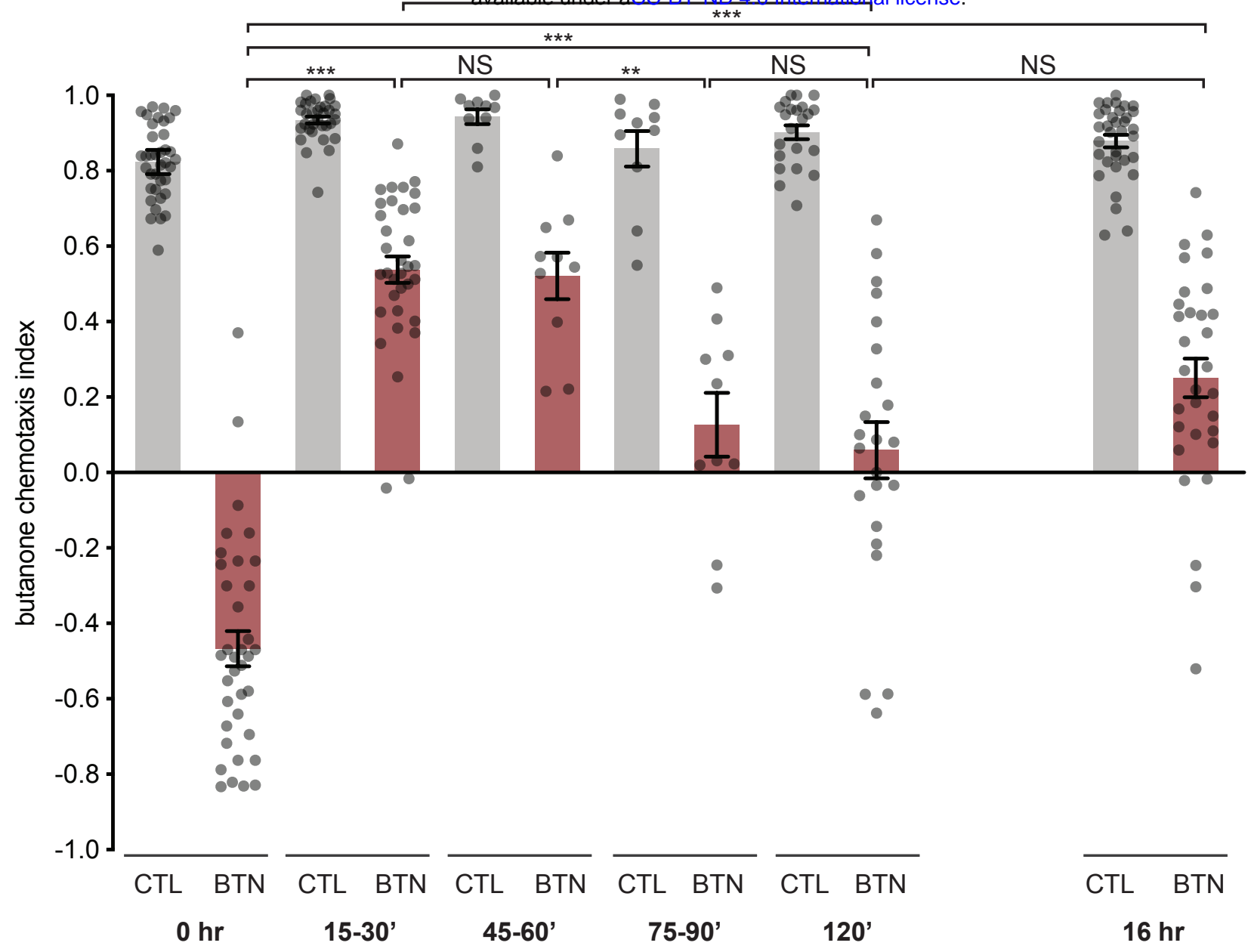

B

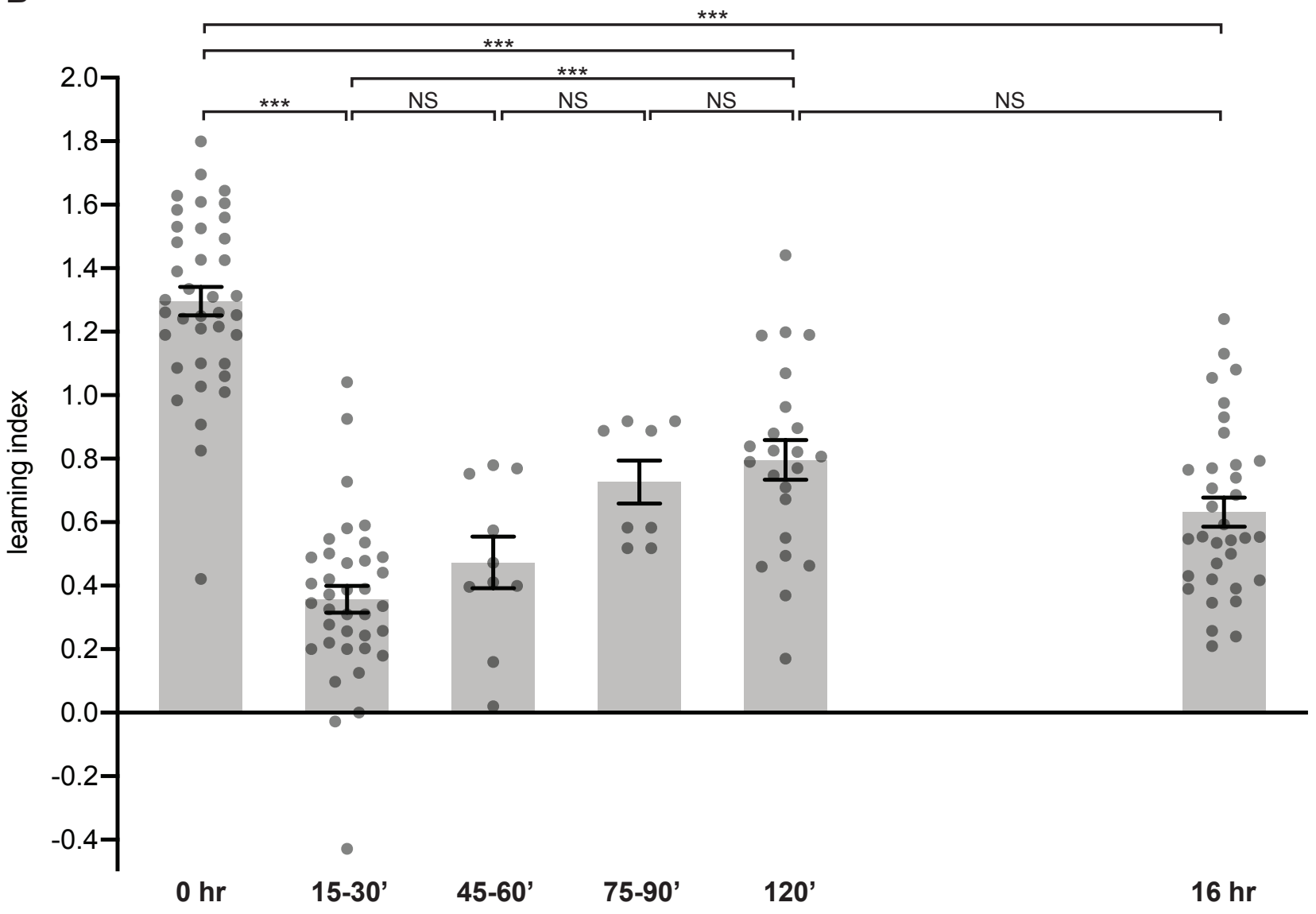


A bioRxiv preprint doi: https://doi.org/10.1101/2020.11.24.395228; this version posted April $\varepsilon$
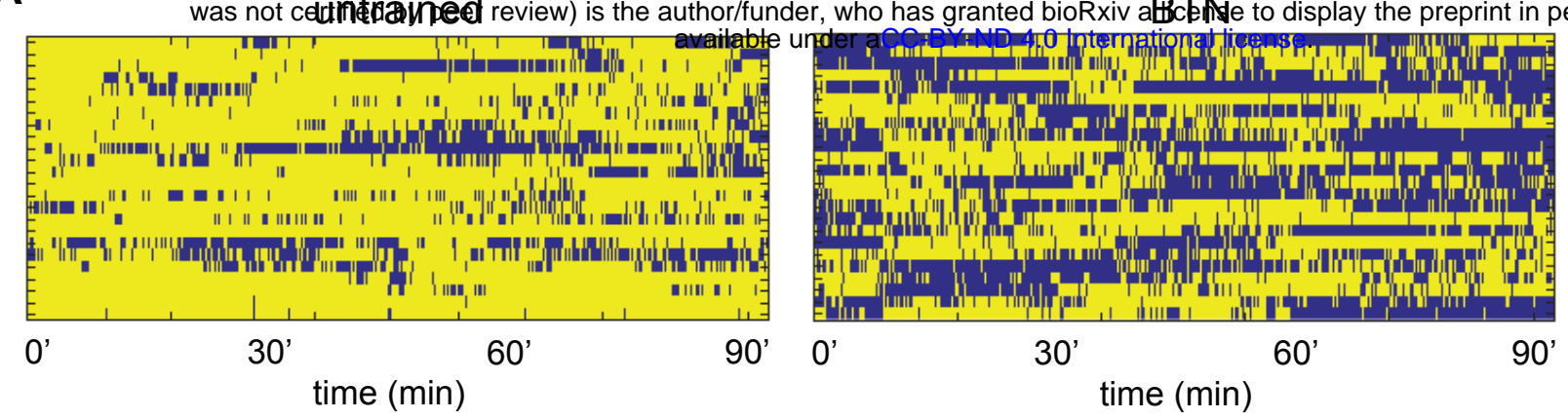

B

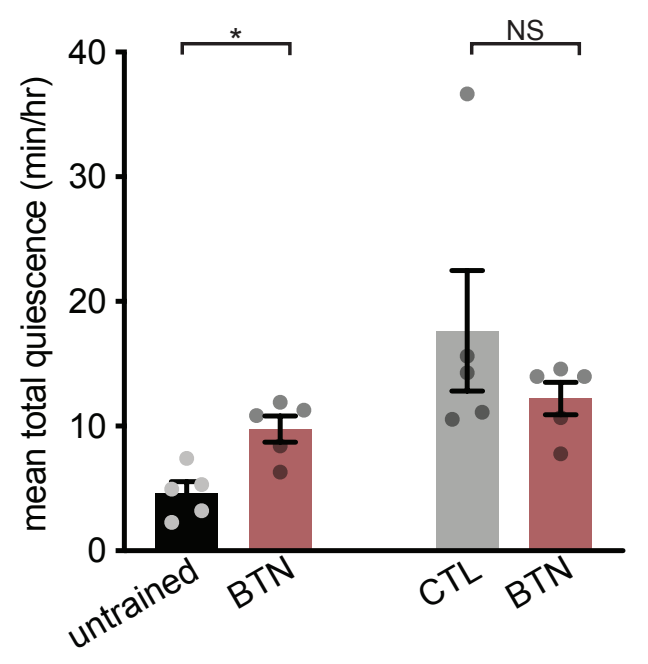

D

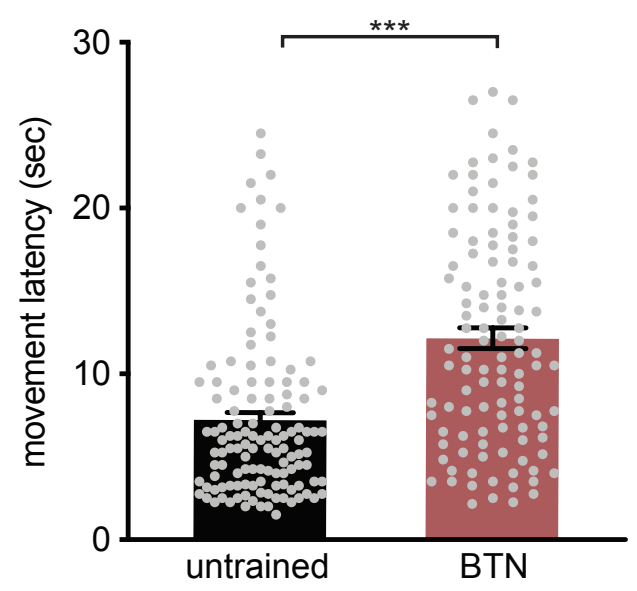

C

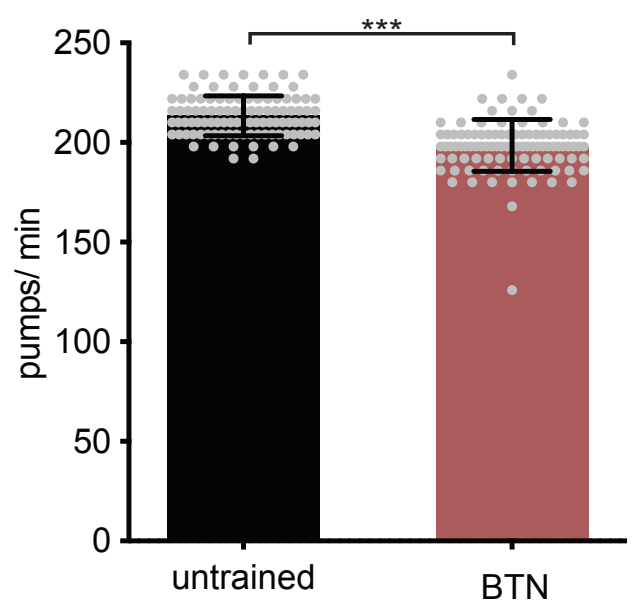

E

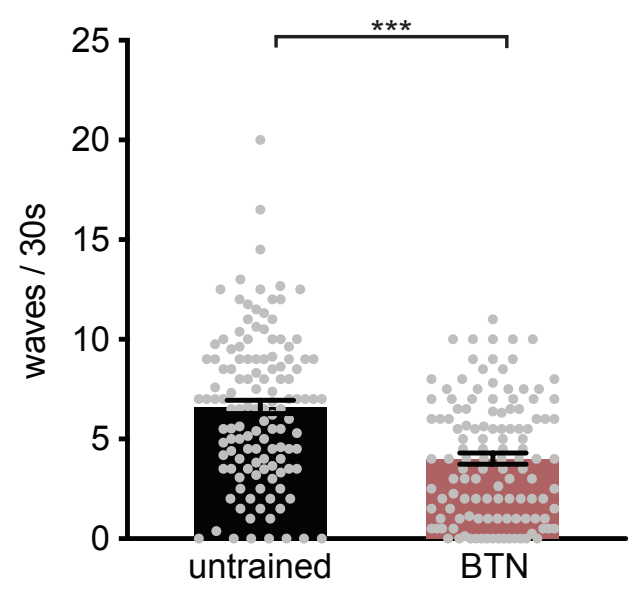


A

bioRxiv preprint doi: https://doi.org/10.1101/2020.11.24.395228; this version posted April

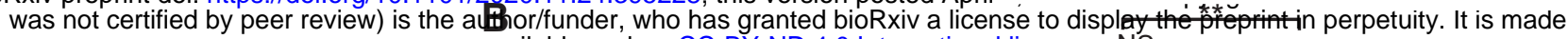

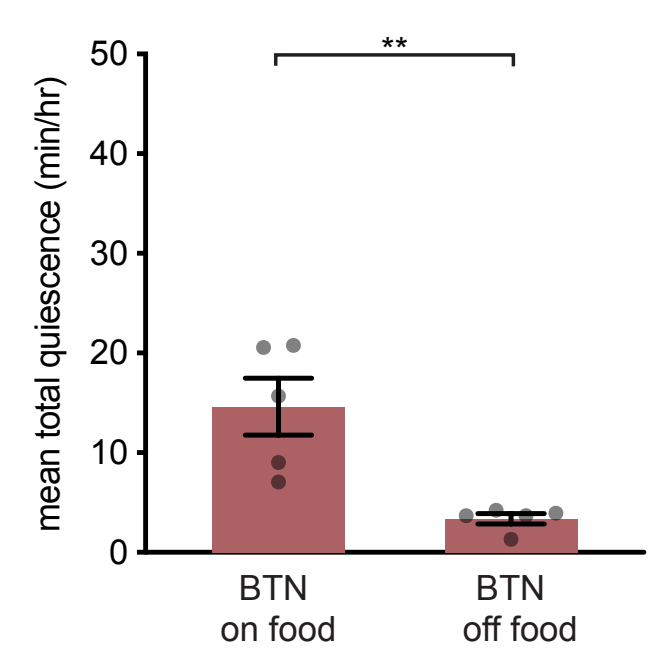

C

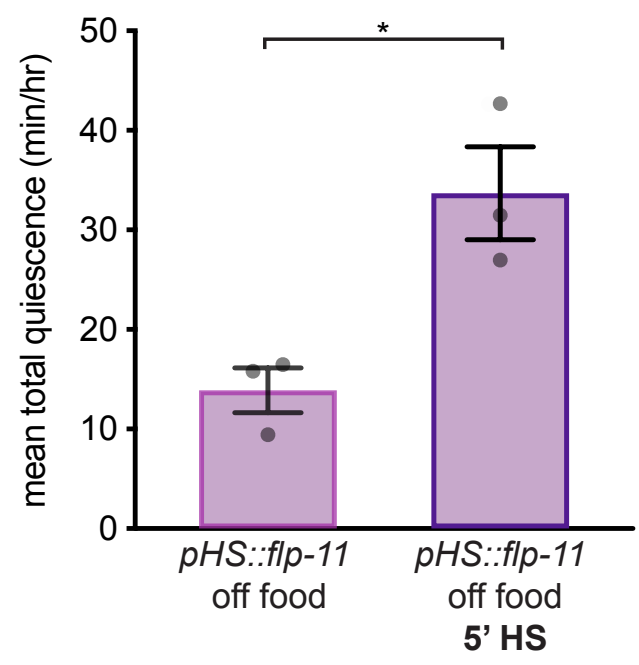

available under aCC-BY-ND 4.0 International license. NS

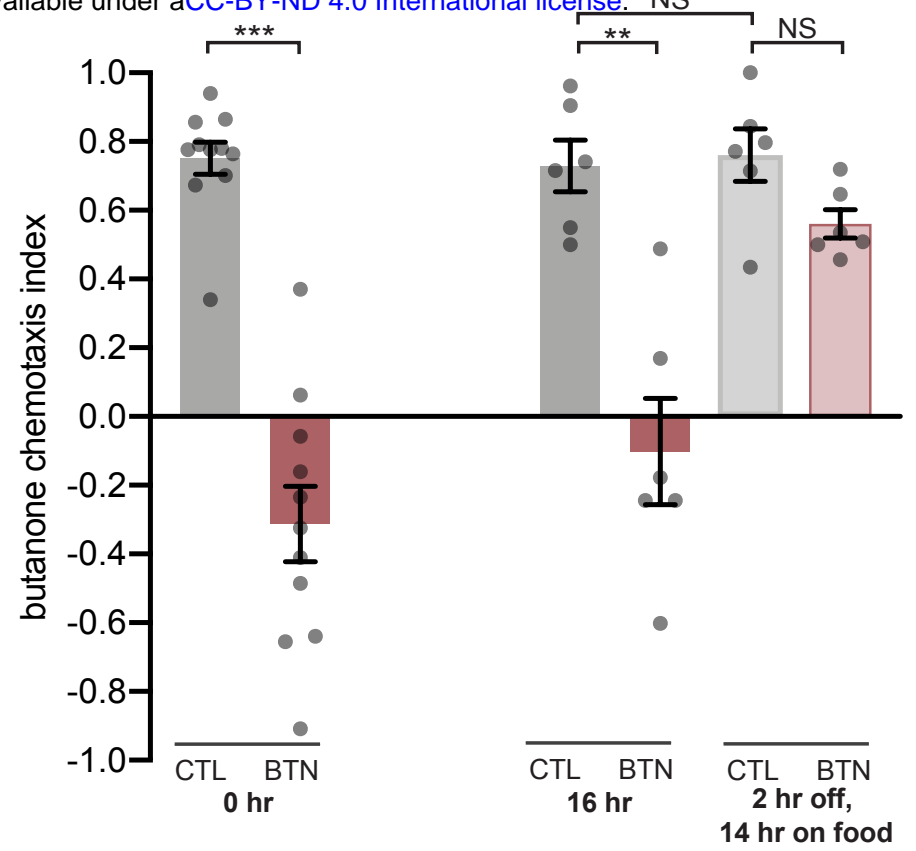

D

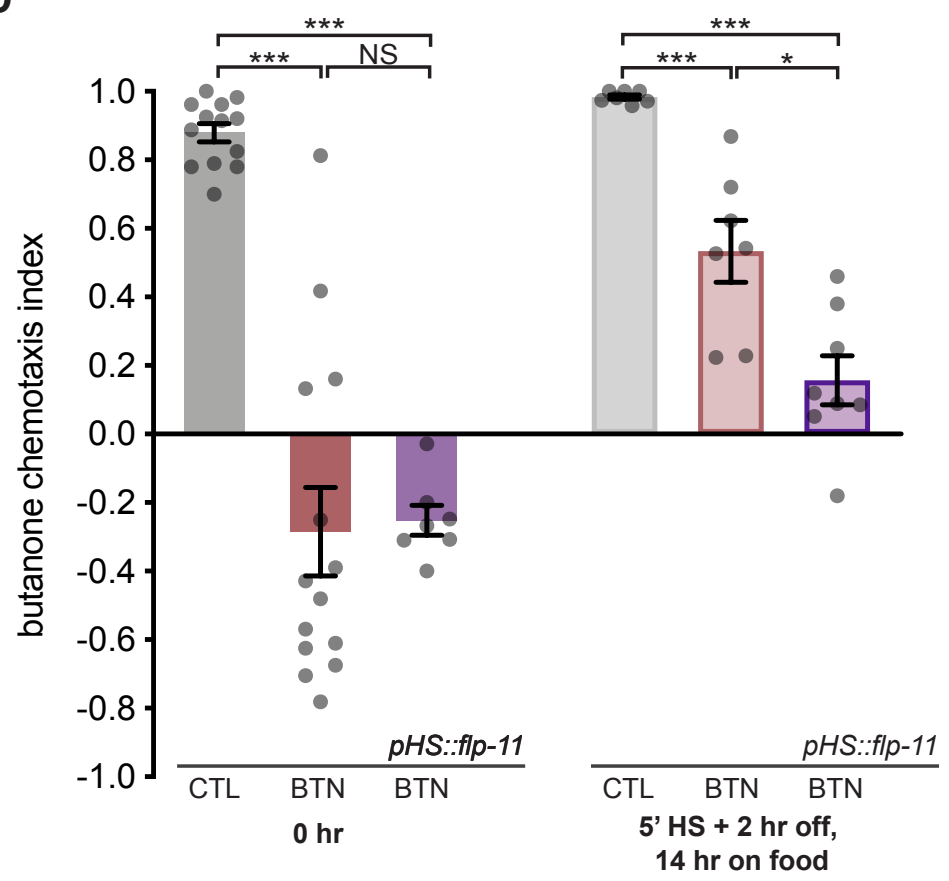




\section{A bioRxiv preprint doi: https://doi.org/10.1101/2020.11.24.395228; this version posted April \&}

was not certified by peerockoriew) is the author/funder, who has granted bioRxiv a license to display the preprint in perpetuity. It is made 1-10 synapses

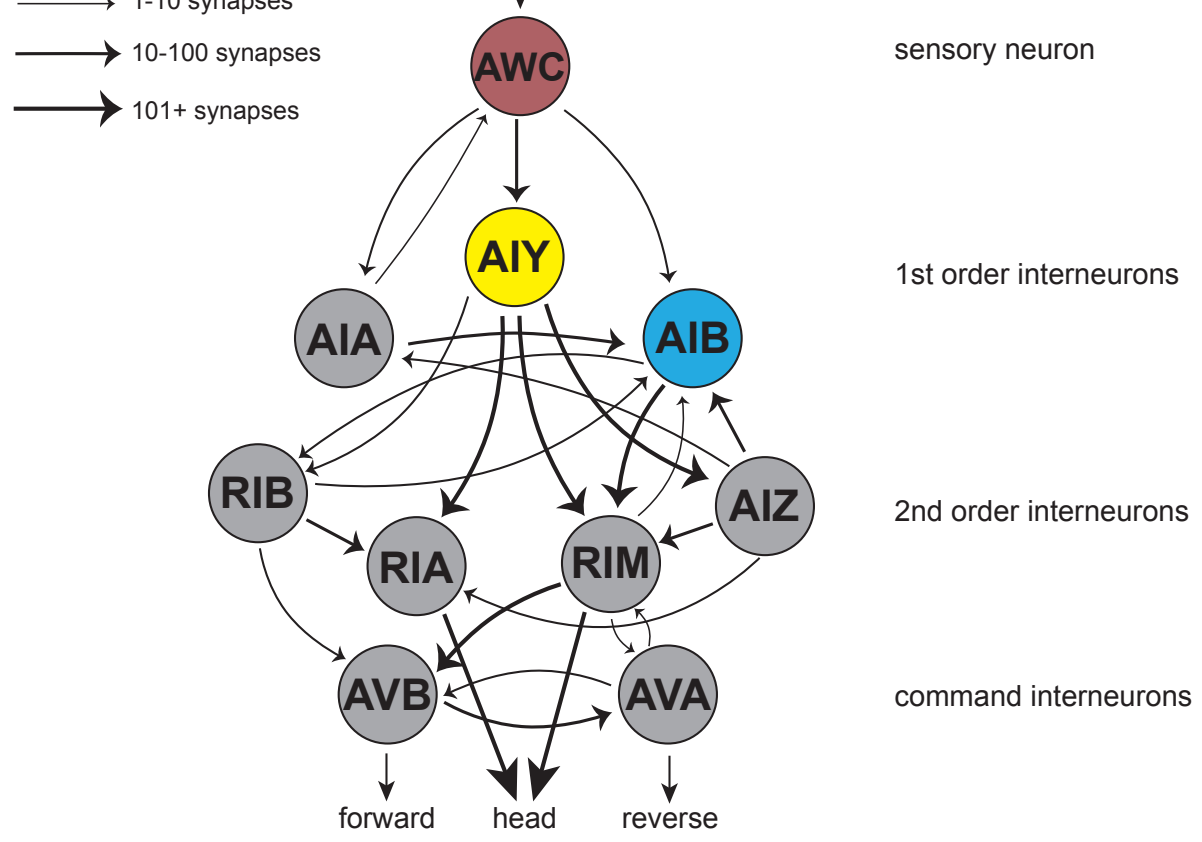
B
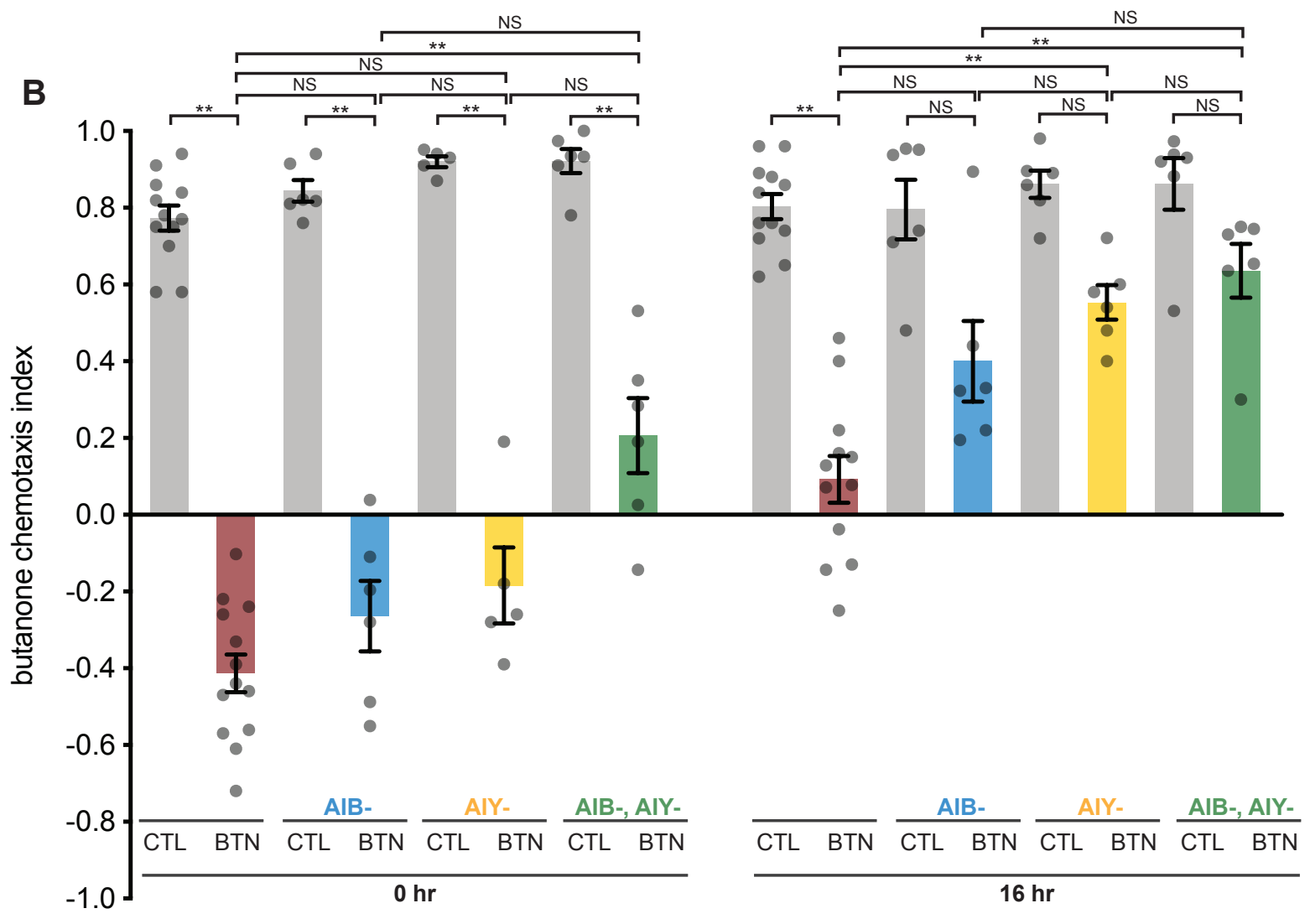
A

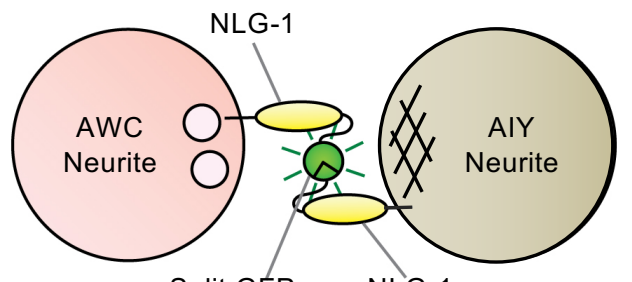

Split GFP
B

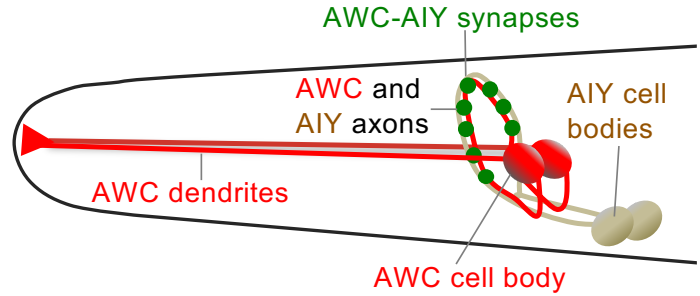

C
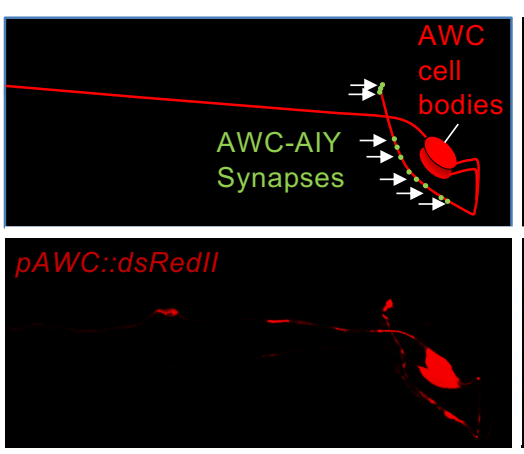

D
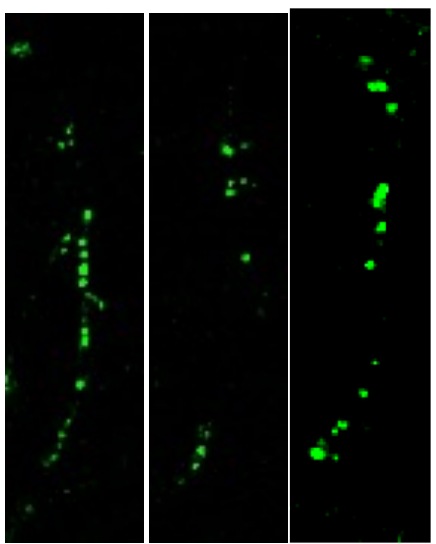

$\frac{\text { CTL }}{16 \mathrm{hr} \text { on food }} \frac{\text { CTL }}{\begin{array}{c}2 \mathrm{hr} \text { off, } \\ 14 \mathrm{hr} \text { on food }\end{array}}$
pAWC::nlg-1::GFP11,

PAIY::nlg-1::GFP1-10
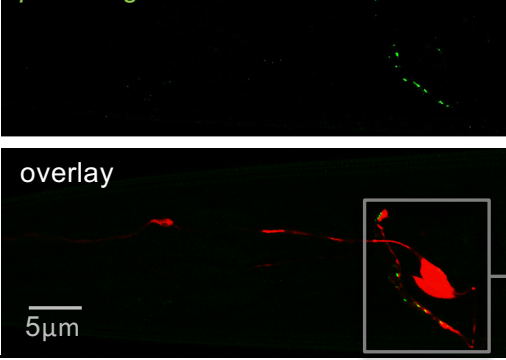

E

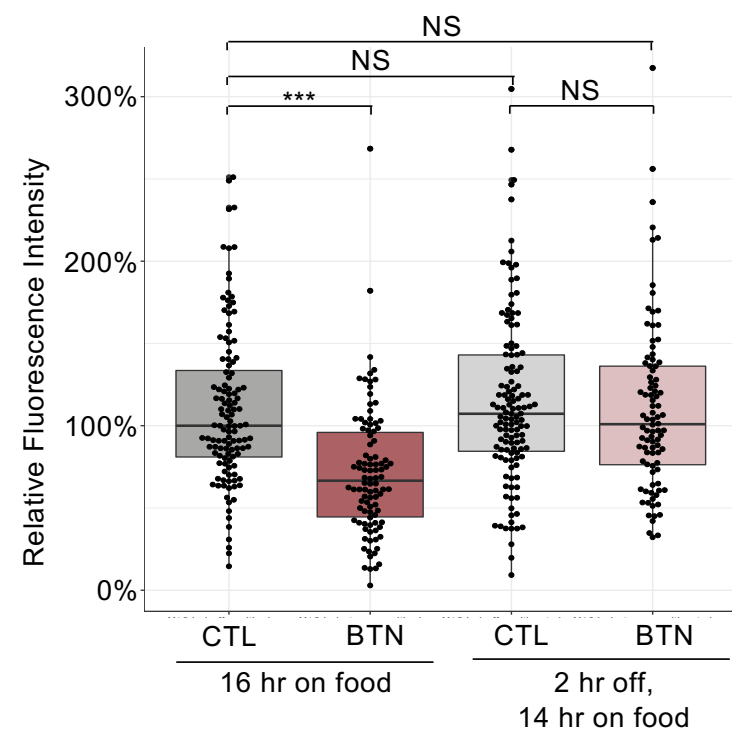

$\mathbf{F}$

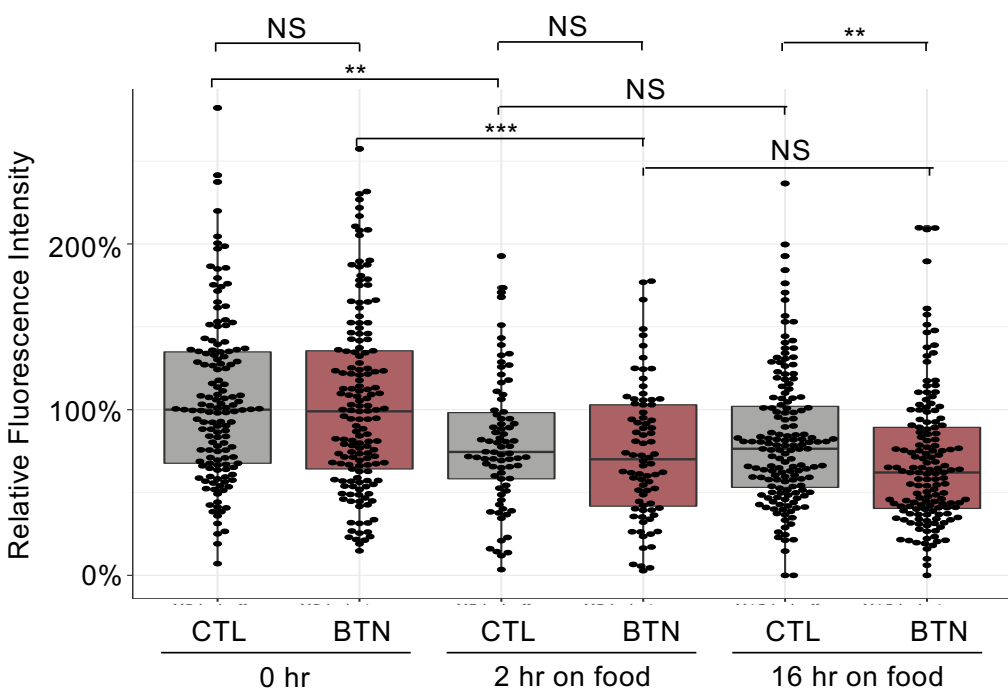




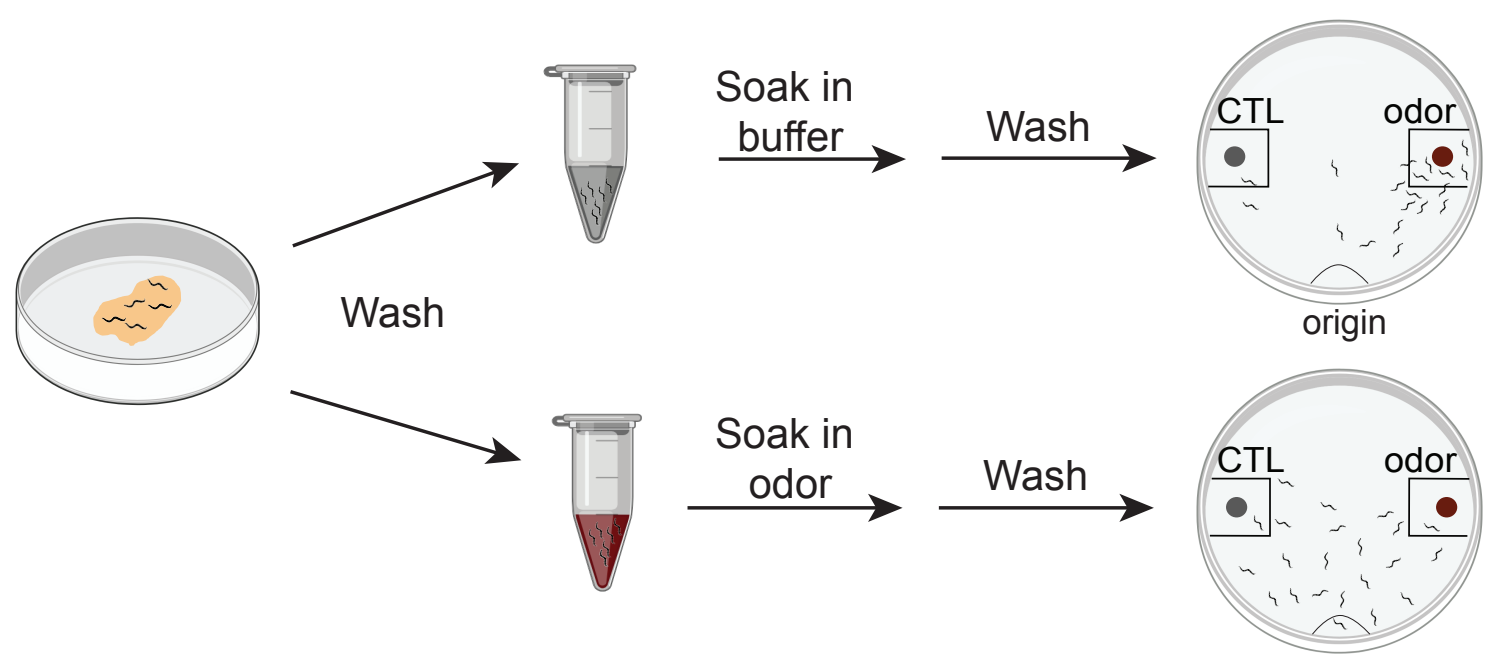

Chemotaxis index $=\frac{(\text { worms at odor })-(\text { worms at control })}{(\text { total worms on plate not at origin })}$ 
bioRxiv preprint doi: https://doi.org/10.1101/2020.11.24.395228; this ve

was not certified by peer review) is the author/funder, who has granteg gioffixiv a license to display the preprint in perpetuity. It is made available under aCC-BY-ND 4.0 International license.

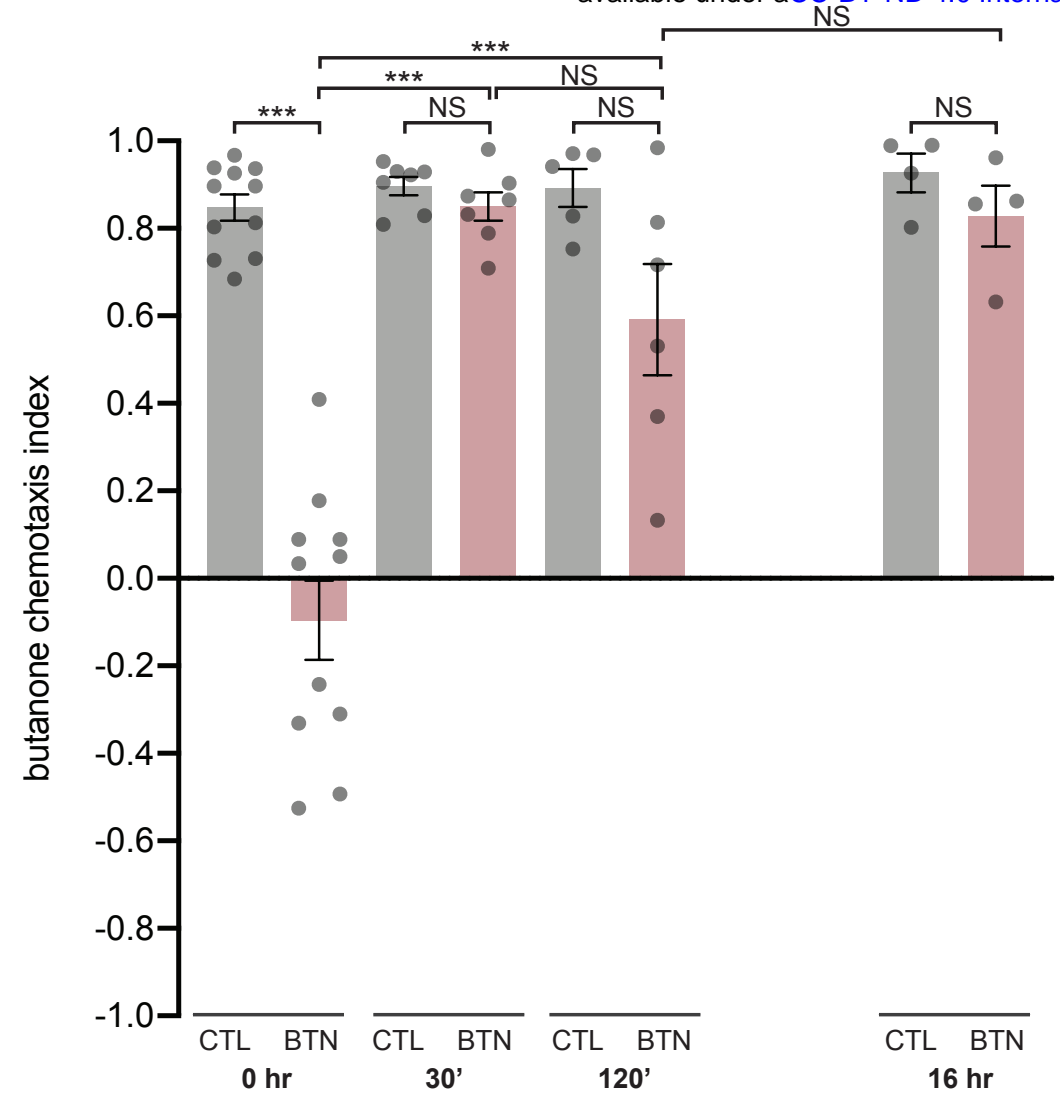



available under aCC-BY-ND 4.0 International license.

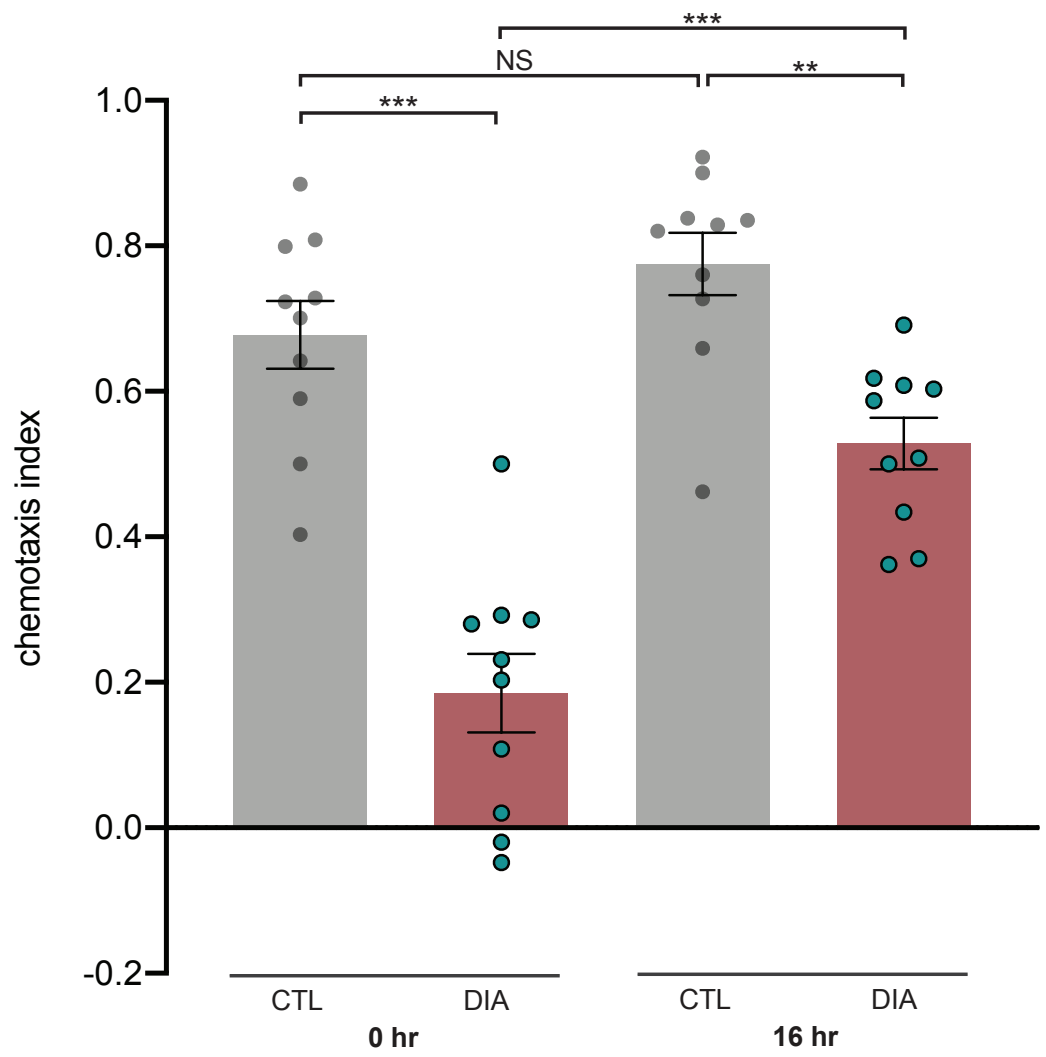


bioRxiv preprint doi: https://doi.org/10.1101/2020.11.24.395228; this ve

was not certified by peer review) is the author/funder, who has grantgethojeffixiv a license to display the preprint in perpetuity. It is made available under aCONY-ND 4.0 International license.

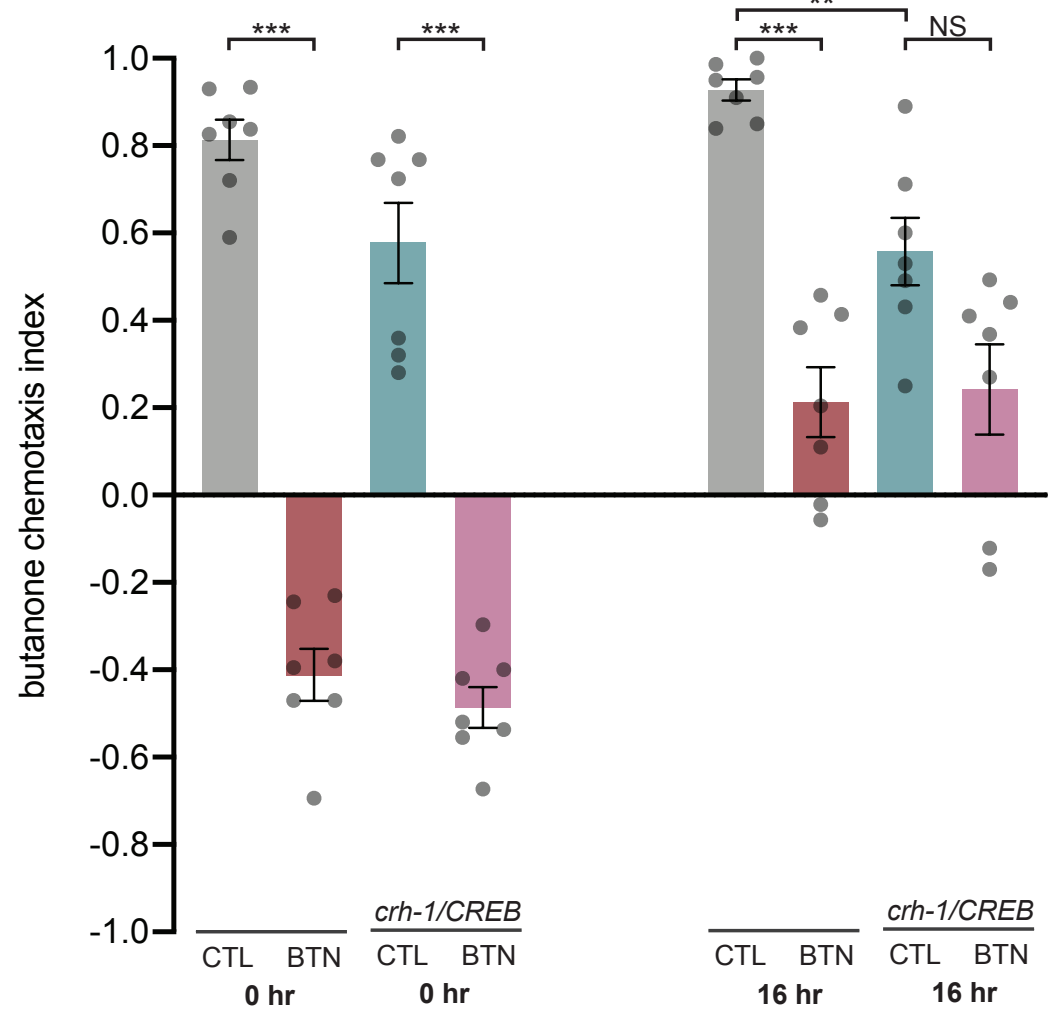


bioRxiv preprint doi: https://doi.org/10.1101/2020.11.24.395228; this ve

was not certified by peer review) is the author/funder, who has grantgsfgioffixiv a license to display the preprint in perpetuity. It is made available under aCC-BY-ND 4.0 International license.

mean total quiescence (min/hr)

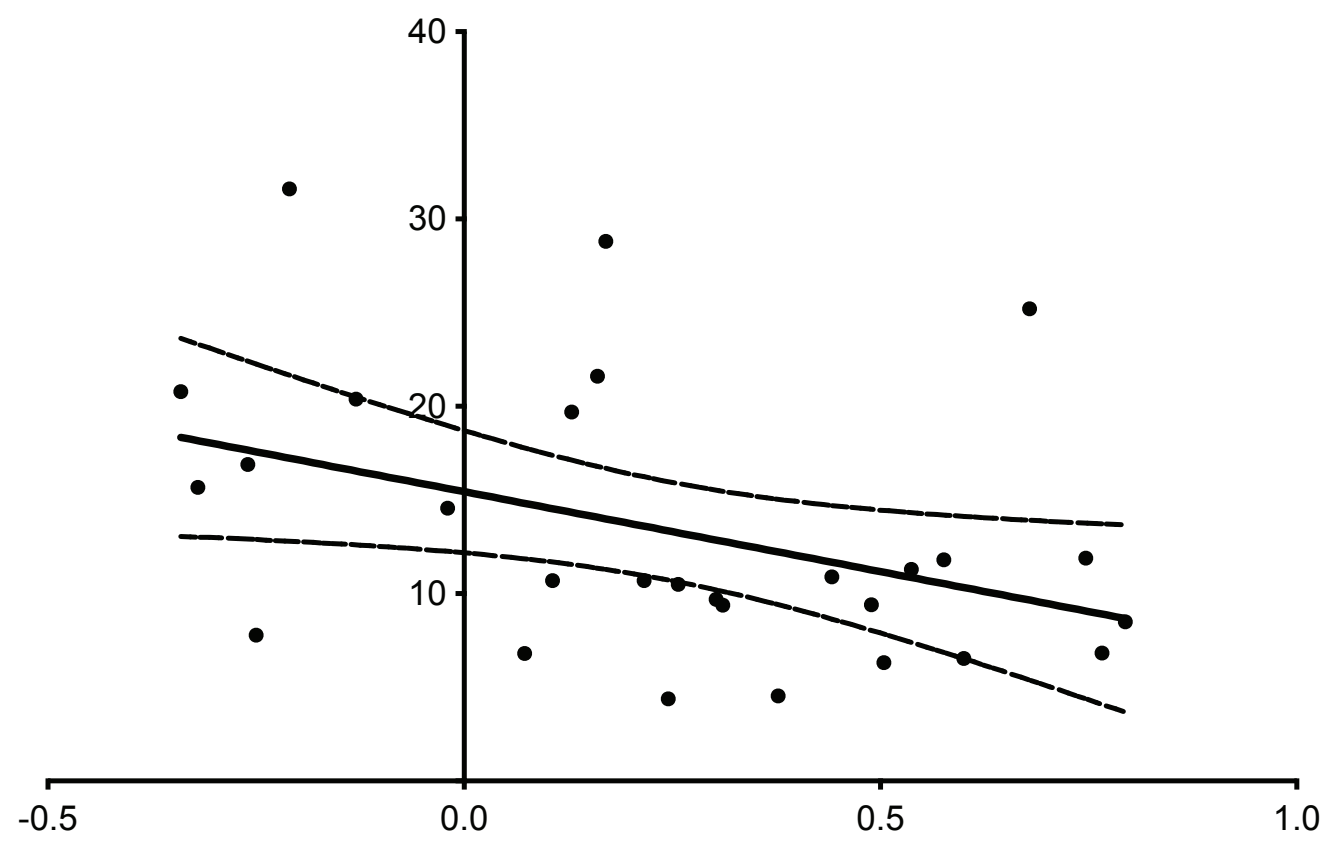

chemotaxis index at 16 hours post training 
A

pPHB::mCherry

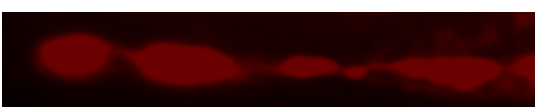

pPHB:::nlg-1::GFP1-10, pAVA::nlg-1::GFP11

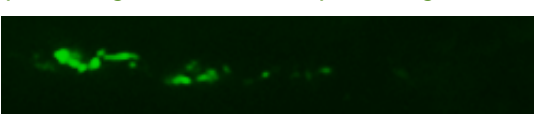

overlay

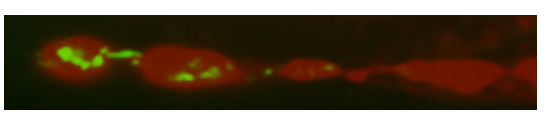

B

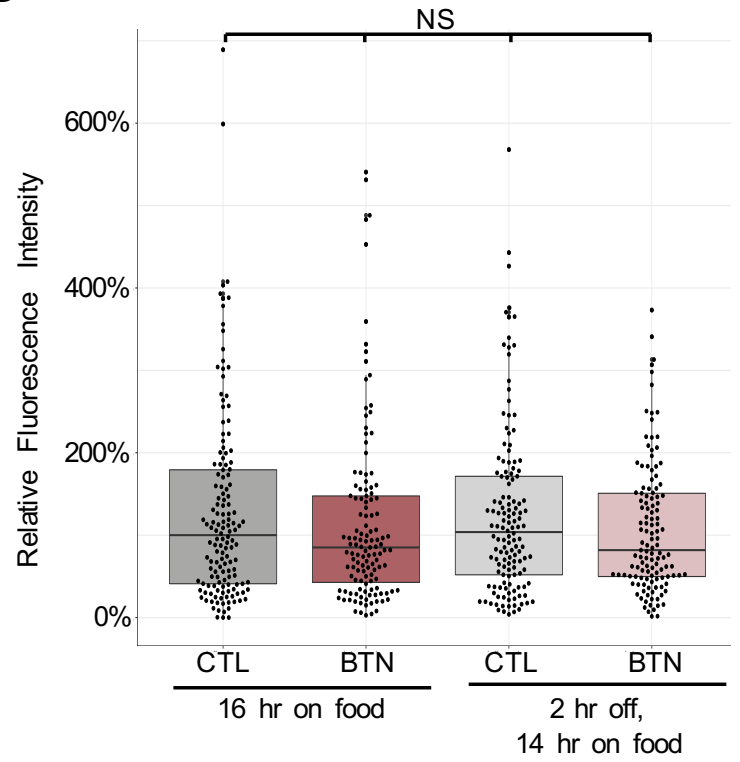


A

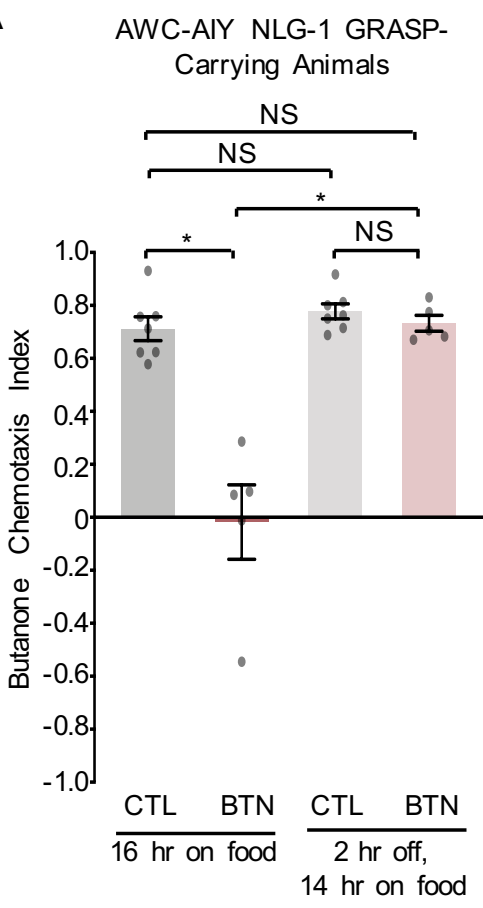

C
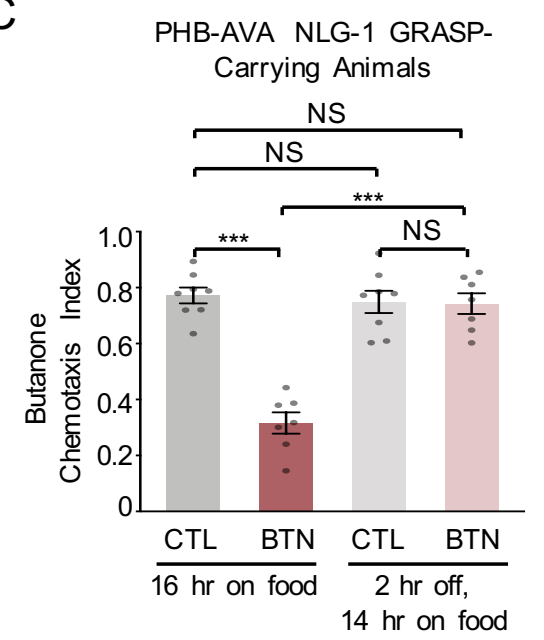

B

AWC-AIY NLG-1 GRASP-

Carrying Animals

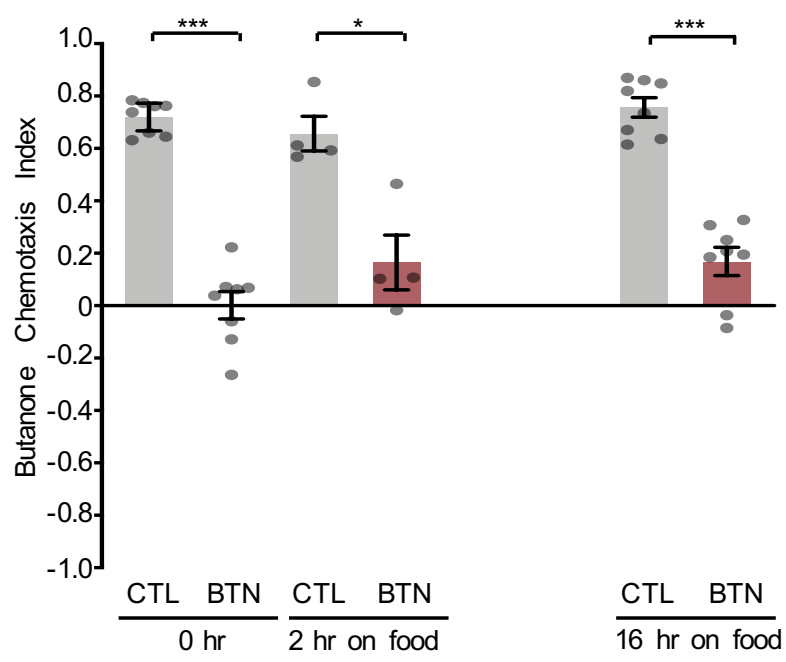

